University of Tennessee Health Science Center

UTHSC Digital Commons

\title{
Identification of Effectors of Synergistic Lethality in Candida albicans-Staphylococcus aureus Polymicrobial Intra-abdominal Infection
}

Olivia Adele Todd

University of Tennessee Health Science Center

Follow this and additional works at: https://dc.uthsc.edu/dissertations

Part of the Bacterial Infections and Mycoses Commons, Biological Phenomena, Cell Phenomena, and Immunity Commons, Disease Modeling Commons, and the Medical Microbiology Commons

\section{Recommended Citation}

Todd, Olivia Adele (https://orcid.org/0000-0002-1092-1984), "Identification of Effectors of Synergistic Lethality in Candida albicans-Staphylococcus aureus Polymicrobial Intra-abdominal Infection" (2021). Theses and Dissertations (ETD). Paper 555. http://dx.doi.org/10.21007/etd.cghs.2021.0537. 


\title{
Identification of Effectors of Synergistic Lethality in Candida albicans- Staphylococcus aureus Polymicrobial Intra-abdominal Infection
}

\begin{abstract}
Candida albicans, an opportunistic fungal pathogen, and Staphylococcus aureus, a ubiquitous pathogenic bacterium, are among the most prevalent causes of nosocomial infections and cause severe morbidity and mortality. Moreover, they are frequently coisolated from central venous catheters and deep-seated infections, including intra-abdominal sepsis. Relatively little is known about the complex interactions and signaling events that occur between microbes and even less so how microbial "cross-talk" shapes human health and disease.

Using a murine model of polymicrobial intra-abdominal infection (IAI), we have previously shown that coinfection with $\mathrm{C}$. albicans and $\mathrm{S}$. aureus leads to synergistic lethality whereas monomicrobial infection is nonlethal. Therefore, we aimed to identify staphylococcal virulence determinants that drive lethal synergism in polymicrobial IAI. Using the toxigenic S. aureus strain JE2, we observed that co-infection with C. albicans led to a striking $80-100 \%$ mortality rate within $20 \mathrm{~h}$ p.i while monomicrobial infections were non-lethal. Use of a GFP-P3 promoter S. aureus reporter strain revealed enhanced activation of the staphylococcal agr quorum sensing system during in vitro polymicrobial versus monomicrobial growth. Analyses by qPCR, Western blot, and toxin functional assays confirmed enhanced agr-associated gene transcription and increases in secreted $\alpha$ - and $\delta$-toxins. C. albicans-mediated elevated toxin production and hemolytic activity was determined to be agrA-dependent and genetic knockout and complementation of hla identified $\alpha$-toxin as the key staphylococcal virulence factor driving lethal synergism. Analysis of mono- and polymicrobial infection $8 \mathrm{~h} \mathrm{p}$.i. demonstrated equivalent bacterial burden in the peritoneal cavity, but significantly elevated levels of $\alpha$-toxin (3-fold) and the eicosanoid PGE2 (4-fold) during coinfection. Importantly, prophylactic passive vaccination using the monoclonal anti- $\alpha$-toxin antibody MEDI4893* led to significantly improved survival rates as compared to treatment with isotype control antibody. Collectively, these results define $\alpha$-toxin as an essential virulence determinant during $C$. albicans-S. aureus IAI and describe a novel mechanism by which a human pathogenic fungus can augment the virulence of a highly pathogenic bacterium in vivo.
\end{abstract}

We next sought to unravel the mechanism by which $\mathrm{C}$. albicans drives enhanced staphylococcal $\alpha$-toxin production. Using a combination of functional and genetic approaches, we determined that an intact agr quorum sensing regulon is necessary for enhanced $\alpha$-toxin production during coculture and that a secreted candidal factor likely is not implicated in elevating agr activation. As the agr system is $\mathrm{pH}$ sensitive, we observed that $\mathrm{C}$. albicans raises the $\mathrm{pH}$ during polymicrobial growth and that this correlates with increased agr activity and $\alpha$-toxin production. By using a $\mathrm{C}$. albicans mutant deficient in alkalinization $(\operatorname{stp} 2 \Delta / \Delta)$, we confirmed that modulation of the extracellular $\mathrm{pH}$ by $\mathrm{C}$. albicans can drive agr expression and toxin production. Additionally, the use of various Candida species (C. glabrata, C. dubliniensis, $\mathrm{C}$. tropicalis, C. parapsilosis, and $\mathrm{C}$. krusei) demonstrated that those capable of raising the extracellular $\mathrm{pH}$ correlated with elevated agr activity and $\alpha$-toxin production during coculture. Overall, we demonstrated that alkalinization of the extracellular $\mathrm{pH}$ by the Candida species leads to sustained activation of the staphylococcal agr system.

Finally, we correlated $\alpha$-toxin production with significant increases in biomarkers of liver and kidney damage during coinfection and determined that functional toxin was required for morbidity and mortality. We next sought to determine the candidal effector(s) mediating this enhanced virulence by employing an unbiased screening approach. C. albicans transcription factor mutants were evaluated for their ability to induce $\mathrm{S}$. aureus agr activation in polymicrobial culture. Incredibly, we identified several mutants that displayed defects in augmenting S. aureus agr activity in vitro. Two of the mutants failed to completely synergize with $\mathrm{S}$. aureus in vivo and further analysis revealed the necessity of the uncharacterized $\mathrm{C}$. 
albicans transcription factor, ZCF13, in driving enhanced toxin production both in vitro and in vivo. Collectively, we identified a novel effector by which $\mathrm{C}$. albicans augments $\mathrm{S}$. aureus virulence and identified a potential mechanism of fungal-bacterial lethal synergism.

\section{Document Type}

Dissertation

Degree Name

Doctor of Philosophy (PhD)

\section{Program}

Biomedical Sciences

\section{Research Advisor}

Brian M. Peters, PhD

\section{Keywords}

Candida, Intra-abdominal Infection, Polymicrobial, Staphylococcus

\section{Subject Categories}

Bacterial Infections and Mycoses | Biological Phenomena, Cell Phenomena, and Immunity | Disease Modeling | Diseases | Medical Microbiology | Medical Sciences | Medicine and Health Sciences 


\section{UNIVERSITY OF TENNESSEE HEALTH SCIENCE CENTER}

DOCTOR OF PHILOSOPHY DISSERTATION

\section{Identification of Effectors of Synergistic Lethality in Candida albicans- Staphylococcus aureus Polymicrobial Intra-Abdominal Infection}

Author:

Olivia Adele Todd
Advisor:

Brian M. Peters, PhD

A Dissertation Presented for The Graduate Studies Council of

The University of Tennessee Health Science Center in Partial Fulfillment of the Requirements for the Doctor of Philosophy degree from

The University of Tennessee

in

Biomedical Sciences: Microbiology, Immunology, and Biochemistry

College of Graduate Health Sciences

June 2021 
Copyright $\odot 2021$ by Olivia Adele Todd All rights reserved. 


\section{DEDICATION}

To my brother, Jacob, for being so patient with me. 


\section{ACKNOWLEDGEMENTS}

First, I would like to sincerely thank my mentor Brian Peters for all of his guidance and encouragement over the past 4 years. Thank you for investing in me, my future, and helping me achieve my scientific goals.

Second, I would like to thank my committee members: Dave Rogers, Elizabeth Fitzpatrick, Glen Palmer, and Mairi Noverr. I appreciate each of you for serving on my committee and supporting my scientific endeavors.

Third, I would like to thank all the members, past and present, of the Peters lab: Marjo Willems, Dave Lowes, Emily Sansevere, Junyan Liu, Zhenbo Xu, Jian Miao, Amanda Vogel, Jabez Fortwendel, Winter Bruner, and Kathy Barker. You all taught me so much and made the lab a wonderful environment to work in. I'd also like to thank the entire Fungal Pathogens Group at UTHSC for being such a collaborative and friendly group.

Finally, I am so grateful for my family and friends who helped me along the way, giving me guidance, encouragement, stress relief, and support in everything I do. I would not be here without you all.

The work presented herein was funded by NIH-NIAID grant R01 Al116025 (BMP and MCN) and the Center for Pediatric Experimental Therapeutics (CPET). 


\begin{abstract}
Candida albicans, an opportunistic fungal pathogen, and Staphylococcus aureus, a ubiquitous pathogenic bacterium, are among the most prevalent causes of nosocomial infections and cause severe morbidity and mortality. Moreover, they are frequently coisolated from central venous catheters and deep-seated infections, including intraabdominal sepsis. Relatively little is known about the complex interactions and signaling events that occur between microbes and even less so how microbial "cross-talk" shapes human health and disease.
\end{abstract}

Using a murine model of polymicrobial intra-abdominal infection (IAI), we have previously shown that coinfection with $C$. albicans and $S$. aureus leads to synergistic lethality whereas monomicrobial infection is nonlethal. Therefore, we aimed to identify staphylococcal virulence determinants that drive lethal synergism in polymicrobial IAI. Using the toxigenic $S$. aureus strain JE2, we observed that co-infection with $C$. albicans led to a striking $80-100 \%$ mortality rate within $20 \mathrm{~h}$ p.i. while monomicrobial infections were non-lethal. Use of a GFP-P3 promoter S. aureus reporter strain revealed enhanced activation of the staphylococcal agr quorum sensing system during in vitro polymicrobial versus monomicrobial growth. Analyses by qPCR, Western blot, and toxin functional assays confirmed enhanced $a g r$-associated gene transcription and increases in secreted $\alpha$ and $\delta$-toxins. $C$. albicans-mediated elevated toxin production and hemolytic activity was determined to be agrA-dependent and genetic knockout and complementation of hla identified $\alpha$-toxin as the key staphylococcal virulence factor driving lethal synergism. Analysis of mono- and polymicrobial infection $8 \mathrm{~h}$ p.i. demonstrated equivalent bacterial burden in the peritoneal cavity, but significantly elevated levels of $\alpha$-toxin (3-fold) and the eicosanoid $\mathrm{PGE}_{2}$ (4-fold) during co-infection. Importantly, prophylactic passive vaccination using the monoclonal anti- $\alpha$-toxin antibody MEDI4893* led to significantly improved survival rates as compared to treatment with isotype control antibody.

Collectively, these results define $\alpha$-toxin as an essential virulence determinant during $C$. albicans-S. aureus IAI and describe a novel mechanism by which a human pathogenic fungus can augment the virulence of a highly pathogenic bacterium in vivo.

We next sought to unravel the mechanism by which $C$. albicans drives enhanced staphylococcal $\alpha$-toxin production. Using a combination of functional and genetic approaches, we determined that an intact agr quorum sensing regulon is necessary for enhanced $\alpha$-toxin production during coculture but that a secreted candidal factor likely is not implicated in elevating agr activation. As the $a g r$ system is $\mathrm{pH}$ sensitive, we observed that $C$. albicans raises the $\mathrm{pH}$ during polymicrobial growth and that this correlates with increased $a g r$ activity and $\alpha$-toxin production. By using a $C$. albicans mutant deficient in alkalinization $(\operatorname{stp} 2 \Delta / \Delta)$, we confirmed that modulation of the extracellular $\mathrm{pH}$ by $C$. albicans can drive agr expression and toxin production. Additionally, the use of various Candida species (C. glabrata, $C$. dubliniensis, $C$. tropicalis, $C$. parapsilosis, and $C$. krusei) demonstrated that those capable of raising the extracellular $\mathrm{pH}$ correlated with elevated agr activity and $\alpha$-toxin production during coculture. Overall, we demonstrated 
that alkalinization of the extracellular $\mathrm{pH}$ by the Candida species leads to sustained activation of the staphylococcal agr system.

Finally, we correlated $\alpha$-toxin production with significant increases in biomarkers of liver and kidney damage during coinfection and determined that functional toxin is required for morbidity and mortality. We next sought to determine the candidal effector(s) mediating this enhanced virulence by employing an unbiased screening approach. C. albicans transcription factor mutants were evaluated for their ability to induce $S$. aureus agr activation in polymicrobial culture. Incredibly, we identified several mutants that displayed defects in augmenting $S$. aureus agr activity in vitro. Two of the mutants failed to completely synergize with $S$. aureus in vivo and further analysis revealed the necessity of the uncharacterized $C$. albicans transcription factor, ZCF13, in driving enhanced toxin production both in vitro and in vivo. Collectively, we identified a novel effector by which $C$. albicans augments $S$. aureus virulence and identified a potential mechanism of fungal-bacterial lethal synergism. 


\section{TABLE OF CONTENTS}

\section{CHAPTER 1. CANDIDA ALBICANS AND STAPHYLOCOCCUS AUREUS PATHOGENECITY AND POLYMICROBIAL INTERACTIONS: LESSONS BEYOND KOCH'S POSTULATES}

Challenging Koch's Postulates: Polymicrobial Interactions ...........................................1

Interkingdom Interactions and Polymicrobial Biofilms ................................................1

Getting the Lines Crossed: Quorum Sensing and Intermicrobial Communication ..........3

Candida albicans and Staphylococcus aureus: Co-Conspirators .................................. 4

Fungal-Bacterial Biofilms and Altered Drug Tolerance..............................................6

Enhanced Pathogenicity in the Oral Cavity and at a Distance.....................................

Polymicrobial Intra-Abdominal Infection and Lethal Synergism...............................10

Mechanisms of Synergistic Lethality: A Role for Staphylococcal Toxins .................11

Lessons Learned, Yet Questions Remain ..................................................................12

\section{CHAPTER 2. CANDIDA ALBICANS AUGMENTS STAPHYLOCOCCUS AUREUS VIRULENCE BY ENGAGING THE STAPHYLOCOCCAL $A G R$ QUORUM SENSING SYSTEM ....................................................................................13}

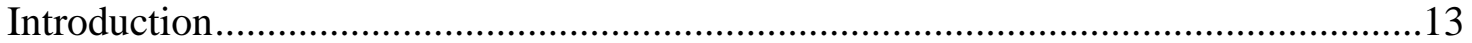

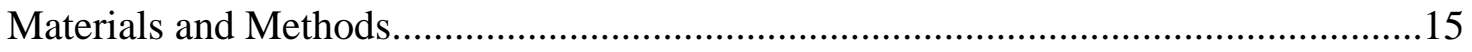

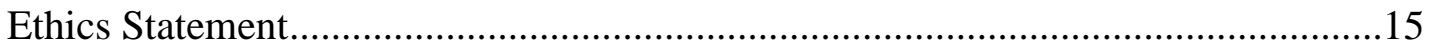

Strains and Growth Conditions ..........................................................................15

Construction of an hla-Complemented Strain .........................................................17

Murine Model of IAI ……............................................................................

Passive Immunization with an Anti- $\alpha$-Toxin Monoclonal Antibody .........................18

Intraperitoneal Delivery of $\alpha$-Toxin...................................................................18

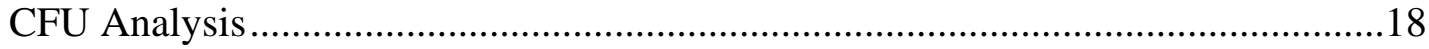

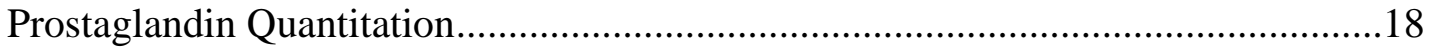

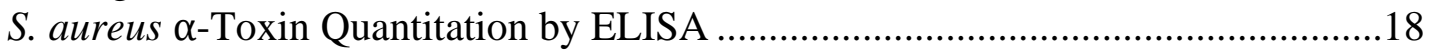

Agar Plate Assay for Toxin Activity ..............................................................19

RBC Hemolytic Assay …………………...................................................19

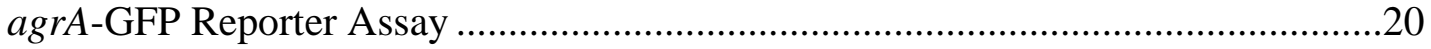

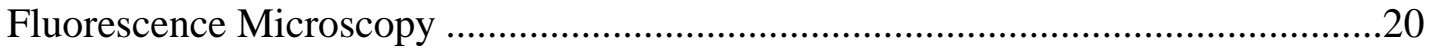

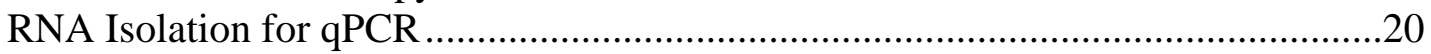

qPCR for Staphylococcal Genes .......................................................................2

Western Blotting for Staphylococcal Proteins ......................................................21

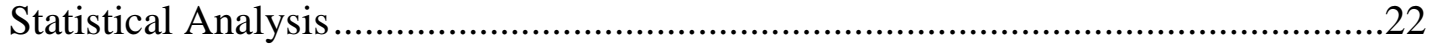

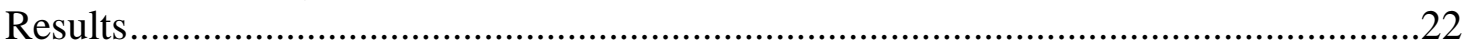

A USA300 Strain Exhibits Robust Synergistic Lethality During Coinfection..........22

The agr Quorum Sensing System Is Enhanced During Coculture .............................22

agr-Regulated Genes and Corresponding Proteins Are Increased During

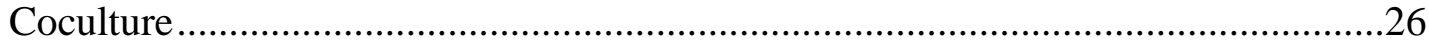

$\alpha$-Toxin Is Required for Lethal Synergism .............................................................28

The $a g r$ System and $\alpha$-Toxin Production Is Increased During Coinfection ...............28 
Passive Immunization with an Anti- $\alpha$-Toxin Antibody Is Protective Against

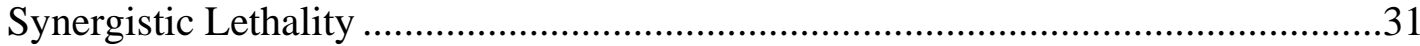

Conclusions

\section{CHAPTER 3. CANDIDA ALBICANS IMPACTS STAPHYLOCOCCUS AUREUS $\alpha$-TOXIN PRODUCTION VIA EXTRACELLULAR ALKALINIZATION ............37}

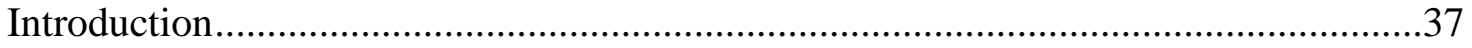

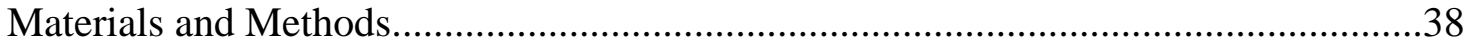

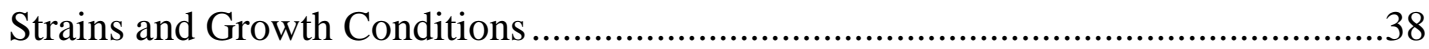

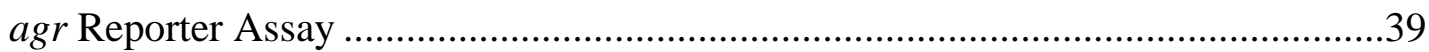

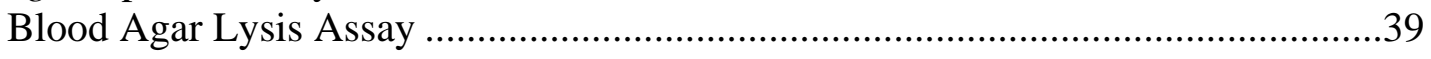

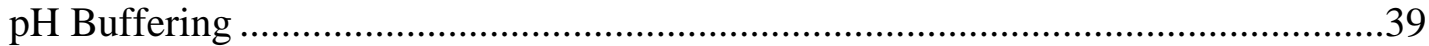

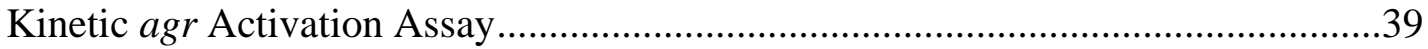

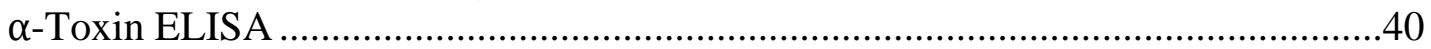

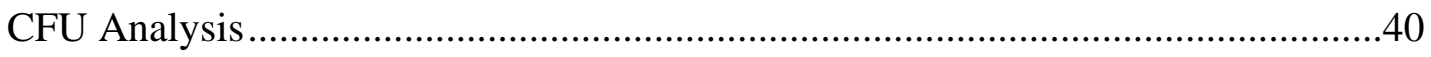

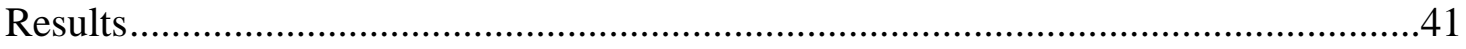

C. albicans Augments Staphylococcal $\alpha$-Toxin and Does Not Complement agr

Signaling in Trans ..............................................................................................4

A Role for Extracellular pH in Modulating agr Signaling During Coculture ...........41

Candida Species Differentially Modulate $\alpha$-Toxin Production During Coculture....46

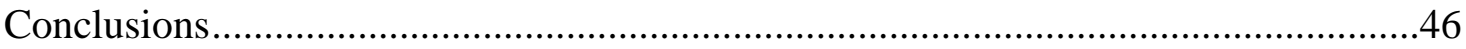

\section{CHAPTER 4. A CRITICAL ROLE FOR THE CANDIDA ALBICANS}

TRANSCRIPTION FACTOR, ZCF13, IN ENHANCING STAPHYLOCOCCUS AUREUS $\alpha$-TOXIN PRODUCTION ....................................................................53

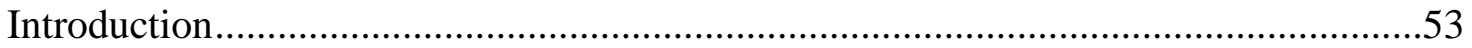

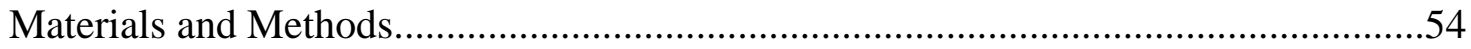

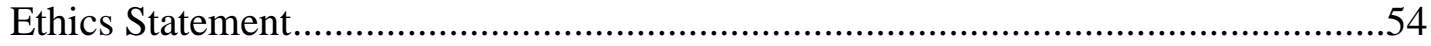

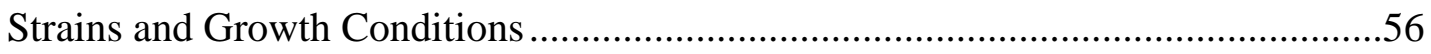

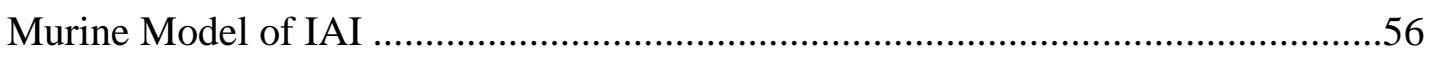

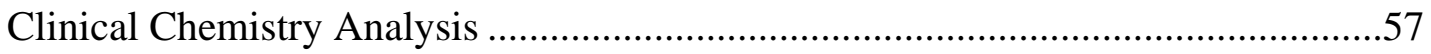

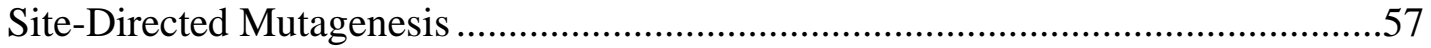

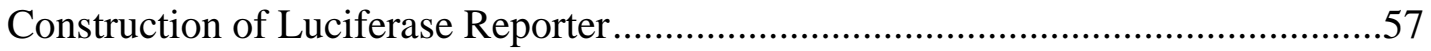

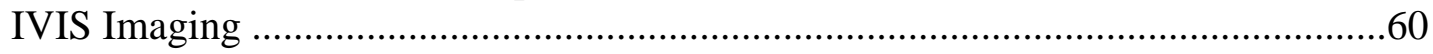

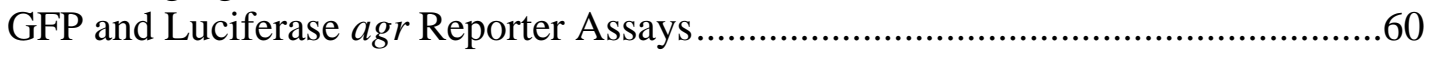

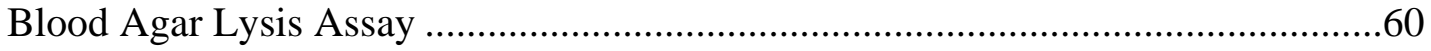

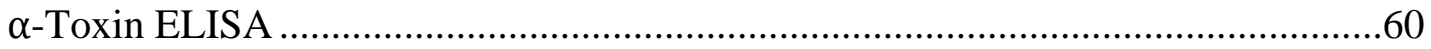

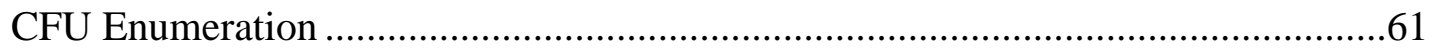

Transcription Factor Mutant Screen ..................................................................61

Plasmid Construction ..................................................................................61

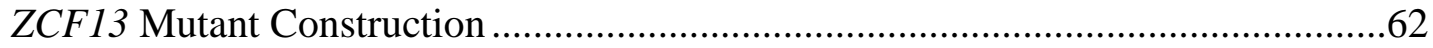

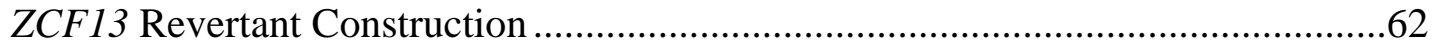

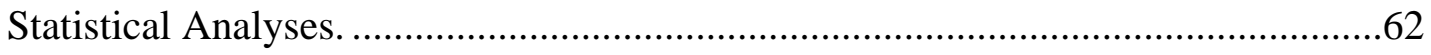

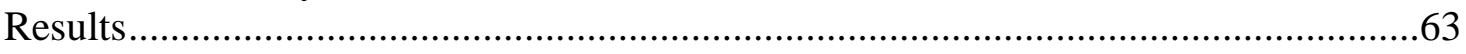

$\alpha$-Toxin Is Responsible for Significant Organ Damage During Polymicrobial IAI..63

$\alpha$-Toxin Must Be Functional for Lethal Synergism 
Several C. albicans Transcription Factors Are Important for Enhancing S. aureus

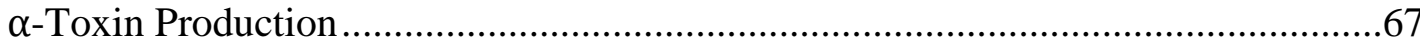

ZCF13 Is Necessary for Candida-Induced agr Activation......................................71

Spatiotemporal Induction of the agr Quorum Sensing System In Vivo.....................71

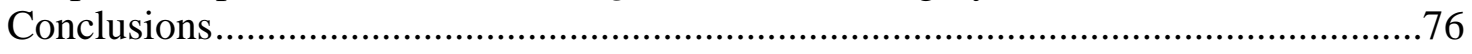

CHAPTER 5. DISCUSSION AND FUTURE DIRECTIONS .........................................80

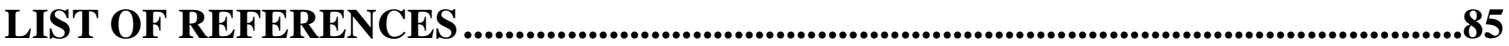

VITA......................................................................................................................................101 


\section{LIST OF TABLES}

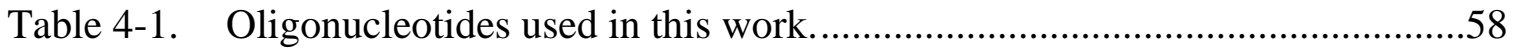

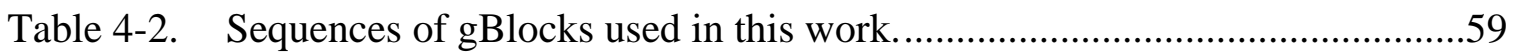

Table 4-3. C. albicans transcription factor mutants identified in screen to have defects in augmenting $S$. aureus agr activity. 


\section{LIST OF FIGURES}

Figure 1-1. Schematic of the agr quorum sensing system in Staphylococcus aureus.......5

Figure 1-2. In vitro polymicrobial biofilm formation by $C$. albicans and $S$. aureus........7

Figure 1-3. Schematic of C. albicans-S. aureus interactions. .....................................

Figure 2-1. S. aureus(pDB22) demonstrates GFP expression in a cell density dependent manner, consistent with predicted agr/P3 promoter activity......16

Figure 2-2. Co-infection with a USA300 strain leads to robust early synergistic mortality.

Figure 2-3. P3-reporter activity, a surrogate of staphylococcal agr activation, and red blood cell lysis is enhanced during co-culture with $C$. albicans.

Figure 2-4. agr-associated genes and staphylococcal toxins are increased during polymicrobial growth with $C$. albicans.

Figure 2-5. Increased $S$. aureus $\alpha$-toxin during growth with $C$. albicans is agrdependent and $\alpha$-toxin is required for lethal infectious synergism during IAI.

Figure 2-6. The S. aureus agr quorum sensing system is engaged during IAI and polymicrobial infection leads to synergistic increases in $\mathrm{PGE}_{2}$ and $\alpha$ toxin.

Figure 2-7. $\alpha$-toxin is necessary but not sufficient to drive infectious synergism during IAI.

Figure 3-1. C. albicans enhances $S$. aureus $\alpha$-toxin production.

Figure 3-2. Candida-enhanced toxin production is dependent on intact staphylococcal agrA and $a g r B$ signaling....

Figure 3-3. Synergistic hemolysis is partially dependent on extracellular $\mathrm{pH}$ and can be overridden by its modulation.

Figure 3-4. The alkalinization-deficient $C$. albicans mutant $\operatorname{stp} 2 \Delta / \Delta$ fails to enhance S. aureus toxin production.

Figure 3-5. Candida spp. differ in their capacity to modulate extracellular $\mathrm{pH}$ and augment toxin production during polymicrobial growth with S. aureus. ....48

Figure 4-1. An alkalinization-deficient mutant shows no defect in synergistic lethality in polymicrobial IAI. 
Figure 4-2. Kinetic changes in organ damage biomarkers.

Figure 4-3. $\quad \alpha$-toxin activity is required for lethal synergism during polymicrobial IAI.

Figure 4-4. Screen of Candida transcription factor mutants reveals novel regulators of $S$. aureus agr induction.

Figure 4-5. $\Delta / \Delta s f l 1$ and $\Delta / \Delta z c f 13$ mutants fail to cause synergistic lethality during polymicrobial infection.

Figure 4-6. Deletion of ZCF13 in SC5314 abrogates S. aureus toxin production and synergistic lethality.

Figure 4-7. Spatiotemporal agr activation during mono- and polymicrobial infection with WT and $\Delta / \Delta z c f 13$.

Figure 4-8. No differences in hyphal formation observed between wild-type and $\Delta / \Delta z c f 13$ strains. 


\section{LIST OF ABBREVIATIONS}

ADAM10

agr

AIP2

$\mathrm{CA}$

CA-MRSA

CFU

DAMPS

ELISA

GFP

HA-MRSA

IAI

ICU

i.p.

LD50

$\mathrm{MAb}$

MIC

MOPS

MRSA

NAC

$\mathrm{PGE}_{2}$

p.i.

PRRs

PSMs

PVL

qPCR

QS

$\mathrm{RBC}$

rot

SA

s.c.

Spp.

TSA

TSB

TSB-g

TSS

TSST

WT

YPD
A disintegrin and metalloprotease 10

Accessory gene regulator

Auto-inducing peptide 2

Candida albicans

Community-acquired methicillin-resistant Staphylococcus aureus

Colony forming units

Danger-associated molecular patterns

Enzyme-linked immunosorbent assay

Green fluorescent protein

Hospital-acquired methicillin-resistant Staphylococcus aureus

Intra-abdominal infections

Intensive care units

Intraperitoneal

Lethal dose, $50 \%$

Monoclonal antibody

Minimal inhibitory concentration

Morpholinepropanesulfonic acid

Methicillin-resistant Staphylococcus aureus

Non-albicans Candida

Prostaglandin E2

Post infection/inoculation

Pattern recognition receptors

Phenol-soluble modulins

Panton-Valentine leukocidin

Quantitative real-time PCR

Quorum sensing

Red blood cell

Repressor of toxin

Staphylococcus aureus

Subcutaneous

Species

Trypticase soy agar

Trypticase soy broth

$0.6 x$ TSB with $0.2 \%$ glucose

Toxic-shock syndrome

Toxic shock syndrome toxin

Wild-type

Yeast-peptone-dextrose 


\title{
CHAPTER 1. CANDIDA ALBICANS AND STAPHYLOCOCCUS AUREUS PATHOGENECITY AND POLYMICROBIAL INTERACTIONS: LESSONS BEYOND KOCH'S POSTULATES*
}

\author{
Challenging Koch's Postulates: Polymicrobial Interactions
}

In 1890, Robert Koch published one of the seminal ideologies of modern microbial pathogenesis, now commonly referred to as "Koch's Postulates". In order to demonstrate causation between microbe and disease, four straightforward principles were proposed: 1) the microbe must be found in all cases of the disease, but not in healthy organisms, 2) the microbe must be isolated from the diseased individual and grown in pure culture, 3 ) the isolated organism must cause disease in a healthy individual, and 4) the microbe must be reisolated and reidentified as the original causative agent (1). Despite the frequent violation of postulate 1, where healthy individuals often asymptomatically carry opportunistic microbes, Koch's postulates have largely led to a global understanding of disease pathogenesis through the prism of a monomicrobial infection model. However, we now know that microbes rarely exist as single species but are often part of complex polymicrobial communities consisting of bacteria, fungi, viruses, and protozoans. Therefore, it is logical to hypothesize that, at times, interactions between these various microbes and the resulting modulation of gene and protein expression profiles drive disease onset and outcome. Thus, it is difficult to satisfy postulates 2 and 3, given that the existence of multiple microbes may be required to elicit similar infectious outcome. Moreover, the complex interplay between host immune status and genetic composition may confer susceptibility to one individual but not recapitulation of the disease in a second (violation of postulates 3 and 4). While Koch's Postulates have served the field of microbiology very well in its early stages, their utility in helping to understand the pathogenesis of polymicrobial infections is limited. Herein, we describe various interactions employed by polymicrobial communities and focus on the fungal pathogen Candida albicans and the bacterium Staphylococcus aureus as prototypical organisms in understanding mechanisms driving exacerbated outcomes during coinfection.

\section{Interkingdom Interactions and Polymicrobial Biofilms}

Bacteria and fungi often occupy the same ecological and biological niches, existing and interacting in various ways to persist, propagate, and prosper. While microbes certainly exist as free-living forms, a majority of microbial life exists as sessile communities coined "biofilms". Biofilms are three-dimensional, structurally complex

*Modified from final submission with open access permission. Todd, O.A.; Peters, B.M. Candida albicans and Staphylococcus aureus Pathogenicity and Polymicrobial Interactions: Lessons beyond Koch's Postulates. J. Fungi 2019, 5, 81.(2) 
communities of one or more microbe, encased in an extracellular matrix consisting largely of polysaccharides (3). These structures can be formed on both biotic or abiotic surfaces and promote microbial consortia development. Biofilms allow for enhanced adhesion to body surfaces, protection from environmental stressors and the host immune system, and often result in increased tolerance to antimicrobial agents. Due to these properties, biofilms are important sources of infection, and the capacity to form a biofilm is considered an important virulence determinant.

With the advent of high-throughput genome sequencing, our knowledge regarding the distinctive biodiversity existing at specific body sites has expanded exponentially. For example, the oral cavity harbors over 700 unique species, the skin is home to approximately 1000 species, and the gut maintains an incredible biomass composed of greater than 1000 species $(4,5)$. Given the constrained biological space these organisms share, physical, metabolic, secreted, and environmental mechanisms are bound to shape their interaction. There are five general types of interactions observed within ecological relationships: competition, predation, commensalism, parasitism, and mutualism (6). These categories can be further simplified by delineating whether the interaction is symbiotic or antagonistic.

Symbiotic relationships can be mutually beneficial for both microbes, favor one microbe, or simply result in a neutral relationship. As mentioned above, the oral cavity is a dynamic environment that plays host to numerous biofilm communities that can be formed on both the mucosa and the tooth surfaces. While a number of different bacterial species can be found in the mouth, Candida species are the dominant fungus of the oral mycobiome (7). Candida spp. interact with Porphyromonas gingivalis, a Gram-negative anaerobic bacterium that is commonly associated with the progression of periodontal disease (8). Bartnicka et al. described the role of $C$. albicans biofilms in establishing an anoxic environment in which $P$. gingivalis can robustly proliferate under such anaerobic conditions, presumably driving gingival inflammation (9). Similarly, C. albicans also demonstrates a mutualistic relationship with Streptococcus mutans - a common causative agent of dental caries. $S$. mutans readily metabolizes dietary sucrose into glucose, which is further rapidly metabolized by $C$. albicans, generating a persistent acidogenic-aciduric microenvironment that promotes synergistic tooth demineralization and caries formation (10-13).

In a competitive relationship, organisms compete for nutrients and resources within a shared environment. An example of this kind of antagonistic relationship is seen between Pseudomonas aeruginosa, a Gram-negative bacterium, and Rhizopus microsporus, an environmental fungus responsible for a majority of mucormycosis cases. Kousser et al. showed that these two microbes compete for iron within wounds; specifically, $P$. aeruginosa secretes siderophores to take up iron, resulting in inhibition of $R$. microsporus growth and germination (14). Although mediated through a different mechanism, $P$. aeruginosa also displays antagonism against $C$. albicans by binding to the fungus and secreting toxic phenazine compounds and homoserine lactones that are capable of killing the fungus and repressing filamentation, respectively $(15,16)$. Multifaceted relationships (such as those described above) likely frequently occur but on a 
much grander scale, given the complexity of consortia on environmental and host surfaces. These interactions undoubtedly shape microbial physiology with significant consequences for both the microbe and host.

\section{Getting the Lines Crossed: Quorum Sensing and Intermicrobial Communication}

Interkingdom cross-talk is another component of the interactions between communities of bacteria and fungi, which is mediated through recognition of signals as part of quorum sensing (QS). QS is a density-dependent communicative signaling system that regulates coordinated gene expression within a population (17). The discovery of QS, first in bacteria and later in lower eukaryotes, such as fungi, reformed the idea that microbes act independently, even when in a community, and are actually quite social (17). QS is evolutionarily useful for microbes by sensing the changing environment and adapting for the good of the community, including aiding in the acquisition and the sharing of nutrients, tolerance to stress and antimicrobials, adaptation to ecological niches, as well as enhancement of virulence in response to other microbial or host organisms. In bacteria, the QS system relies on an accumulated signal molecule that is produced and recognized by cells, and it is only at certain concentrations of this signal that gene expression is modulated. Many of the signaling molecules are unique to specific species and sometimes even to certain clades within species. For example, there exist four divergent agr (accessory gene regulator) quorum sensing systems in Staphylococcus aureus that produce different signaling molecules (AIP-I, II, III, and IV). These signals, although structurally very similar, exhibit cross-inhibition, or interference, to the other $a g r$ types. This $a g r$ interference leads to inhibition of transcription of the $a g r$ locus of another type, perhaps providing a competitive advantage for the dominant strain (18). Conversely, there is some evidence of degenerate signals and promiscuous receptors, allowing for interspecies cross-talk (19). One study looked at the selectivity of QS receptors across a number of bacterial species and discovered a range of recognition of receptors to non-native quorum signals. Quantitative scoring of interactions took into account the sensitivity of the receptor to the signal as well as the degree of activation upon recognition. The RhlR QS receptor in P. aeruginosa is extremely selective for its own quorum signal and showed the highest score in these experiments. On the other side of the spectrum, the Btar2 receptor of Burkholderia thailandensis responded strongly to several different QS signal molecules, including those from $P$. aeruginosa and Vibrio fischeri. Based on these observations, the Btar2 receptor was defined as promiscuous (19). In addition to bacteria, fungi also undergo QS (20). A large body of research exists focusing on the $C$. albicans QS molecule farnesol, a byproduct of sterol synthesis that represses filamentation without disrupting growth rate $(21,22)$. Interestingly, farnesol also impacts susceptibility to antimicrobials or cell signaling against several bacterial species, including $S$. aureus and $P$. aeruginosa (23-26). At high concentrations, farnesol prevents $S$. aureus biofilm formation partially by damaging the bacterial membrane, and it dose-dependently inhibits production of the carotenoid pigment staphyloxanthin, conferring paradoxical resistance to oxidative stressors by increasing biosynthesis of antioxidant enzymes $(27,28)$. While quorum sensing systems exist in other fungal species, 
the specific molecules have not been identified but appear to be linked to control of dimorphism (29).

The most well-described and studied bacterial QS system in S. aureus is the accessory gene regulatory (agr) system (17, 30-32) (Figure 1-1). It consists of two divergent promoters, $\mathrm{P} 2$ and $\mathrm{P} 3$, that drive expression of two separate transcripts, RNAII and RNAIII, respectively. RNAII encodes for four genes, $\operatorname{agrA}$, $\operatorname{agr} B, \operatorname{agrC}$, and $\operatorname{agrD}$. AgrA and AgrC make up a two-component system where AgrA is the response regulator that, when phosphorylated by the membrane-bound histidine kinase AgrC, activates the $\mathrm{P} 2$ promoter. AgrD is the pre-signal peptide that is modified and secreted through the membrane-bound AgrB as the mature autoinducing peptide 2 (AIP-2). AIP-2 is then recognized by AgrC, completing the signaling circuit. Alternatively, AgrA can also activate the $\mathrm{P} 3$ promoter, driving expression of RNAIII - the effector of the quorum sensing system. RNAIII directly encodes for $\delta$-toxin at its 5 ' end. The 3 ' end contains a consensus sequence that is complementary to the 5 ' end of a number of staphylococcal adhesin genes and, when in complex with such transcripts, prevents their translation by masking the Shine-Dalgarno ribosomal binding site. Via this same mechanism, RNAIII also inhibits the translation of the repressor of toxin (rot) transcriptional regulator, allowing for increased production of toxins. Thus, QS signals (such as those produced by agr system) are imperative in facilitating information exchange between microbial self and non-self.

\section{Candida albicans and Staphylococcus aureus: Co-Conspirators}

C. albicans is a polymorphic fungus that exists as an opportunistic pathogen, colonizing the gut and the mucosa of humans, and is one of the most prevalent human fungal pathogens (33). The capacity to transition between yeast and invasive hyphal morphologies is considered to be its key virulence attribute. The shift from commensal to pathogen is usually due to changes in the immune status of the host. C. albicans can cause superficial infections (e.g., candidiasis) of mucosal sites such as the mouth and the vagina (34). Oral and vaginal candidiasis are the result of an overgrowth of the yeast and are associated with robust inflammation of the mucosal surface $(34,35)$. A more serious infection is candidemia, a systemic infection where Candida invades local tissue, accesses the vasculature, and disseminates throughout the body via the bloodstream.

S. aureus is a Gram-positive coccus bacterium that can cause a variety of different diseases ranging from skin and soft-tissue infections to severe bacteremia and sepsis (36). The pathogenicity of $S$. aureus is primarily mediated through toxin production, which is intimately linked to its quorum sensing system, described in detail above. S. aureus produces a number of toxins: the membrane-damaging and cytolytic toxins $\alpha$ - and $\delta$ toxin, Panton-Valentine leukocidin (PVL), and phenol-soluble modulins (PSMs) and the superantigen toxic shock syndrome toxin (TSST), among numerous others (37). Aside from damage-inducing toxin production, S. aureus can also perturb hemostasis through manipulation of the clotting cascade, resist high levels of antimicrobials due to robust biofilm formation, and avoid clearance by immune cells through capsular polysaccharide 


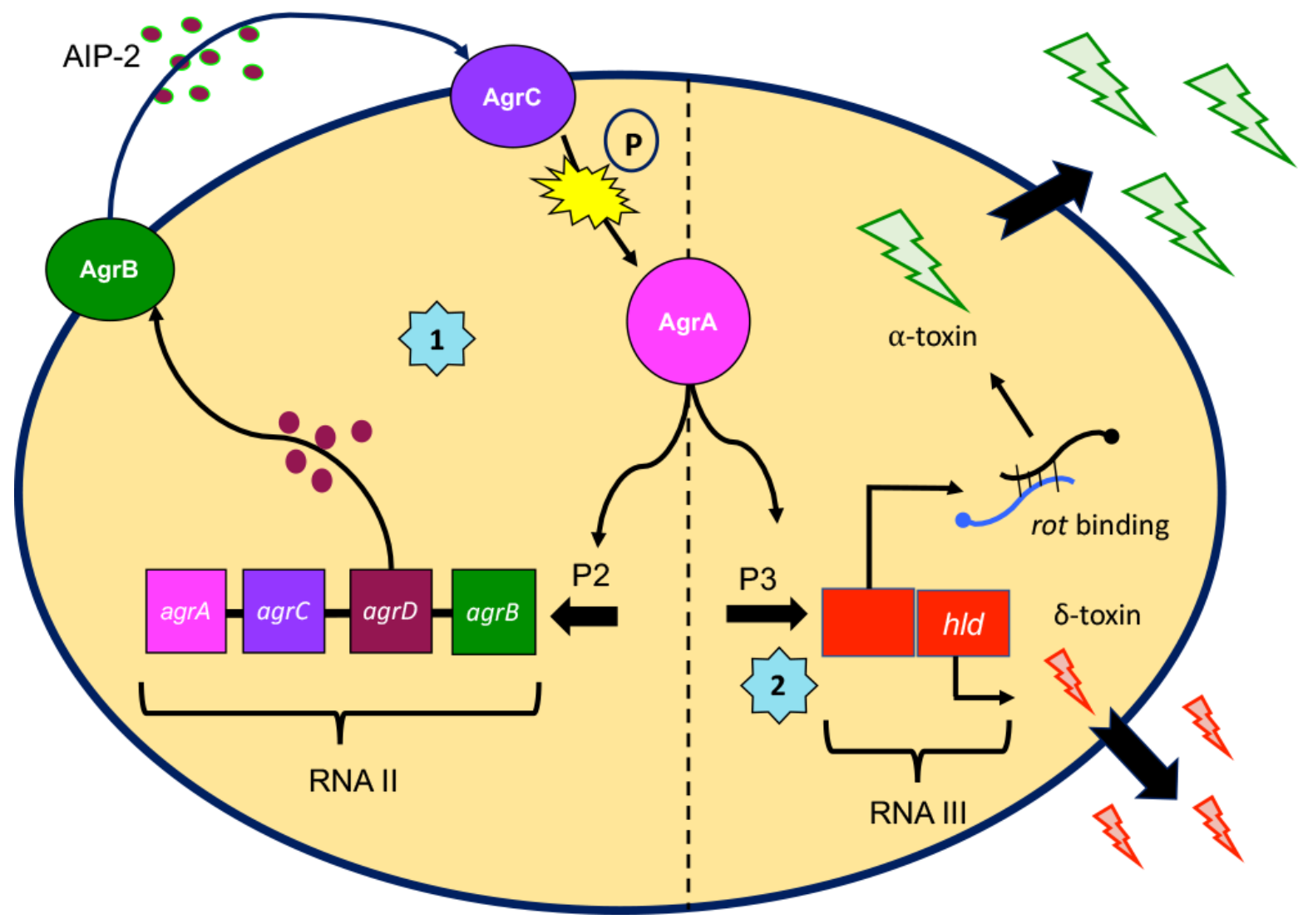

Figure 1-1. Schematic of the agr quorum sensing system in Staphylococcus aureus. AgrA is the response regulator in a two-component system that acts as a transcription factor, modulating toxin production. (1) AgrA activates transcription from the P2 promoter, driving expression of RNAII, an operon consisting of four agr genes. AgrB is a membrane-bound permease that processes AgrD, a pre-signal peptide, and releases it as AIP-2 (auto-inducing peptide 2). AIP-2 is sensed by AgrC, a membrane-bound histidine kinase that is part of the two-component signaling system. AgrC phosphorylates AgrA, activating it, leading to a positive feedback loop. (2) Activated AgrA also drives transcription from the P3 promoter, driving expression of RNA III, the effector of the QS system. RNA III directly encodes for $\delta$-toxin $(h l d)$ and also binds to repressor of toxin (rot) transcript, allowing for toxin production by inhibiting rot translation. RNAIII also binds to a number of adhesin-related genes to similarly block their translation. 
production (38-40). The multi-faceted and redundant nature of $S$. aureus virulence makes it one of the most formidable human pathogens.

While $C$. albicans and $S$. aureus cause significant morbidity and mortality independently, these microbes are also commonly found together at various body sites and are implicated in a variety of diseases, including cystic fibrosis, ventilator-associated pneumonia, urinary tract infections, superinfection of burn wounds, denture stomatitis, and keratitis (41-44). Within the past decade, several in vitro and in vivo animal models have revealed interesting clues as to how these pathogens may cooperate within the host to exacerbate pathogenicity and disease.

\section{Fungal-Bacterial Biofilms and Altered Drug Tolerance}

Polymicrobial infections with $C$. albicans and $S$. aureus are common, due in part to shared niches within the body, including co-isolation from skin, axillae, vagina, pharynx, nasal passages, and oral mucosa (45). C. albicans and S. aureus both have the ability to form biofilms and thus are commonly found growing in polymicrobial biofilms on indwelling medical devices, such as catheters (Figure 1-2). These biofilms are difficult to treat with antimicrobials, as the complex structure of the biofilm protects the organisms by impeding drug permeability and immune cell access. Unfortunately, treatment often involves replacing the catheter, which can be life-threatening in patients with limited options for catheter reinsertion.

Among the earliest work to investigate the development of polymicrobial biofilms by these species revealed that large staphylococcal aggregates formed around hyphal filaments of $C$. albicans, and that $S$. aureus preferentially favored binding to these hyphal filaments as compared to round yeast cells $(46,47)$ (Figure 1-3). Work by Harriott and Noverr demonstrated that $S$. aureus displayed tremendous tolerance to vancomycin [over 1000-fold higher than the planktonic minimal inhibitory concentration (MIC)] during biofilm growth with $C$. albicans. Follow-up studies revealed that viable $C$. albicans was required for this phenotype, and that $S$. aureus became coated with dense extracellular material during polymicrobial growth (46). Coating of $S$. aureus with isolated fungal matrix polysaccharides (as evidenced by increased concanavalin A staining) revealed that elevated antimicrobial tolerance was driven by encasement of bacteria by the dense fungal extracellular meshwork. Additional studies revealed that $C$. albicans mutants (e.g., efg $1 \Delta / \Delta / \operatorname{cph} 1 \Delta / \Delta$ ) unable to adhere to the substratrum due to hyphal growth defects were unable to augment vancomycin tolerance in $S$. aureus (48). Global or specific genetic deletion of adhesins did not seemingly impact vancomycin tolerance profiles during coculture. Newer work by Kong et al. using both genetic and enzymatic approaches to modulate matrix components coupled with fluorescence microscopy-based drug diffusion assays identified the fungal polysaccharide $\beta$-1,3-glucan as the key moiety impeding vancomycin penetration of the biofilm structure (49). This phenomenon of enhanced staphylococcal drug tolerance during polymicrobial biofilm formation is not vancomycinspecific, as reduced susceptibility to doxycycline, nafcillin, and oxacillin has also been observed (49). Moreover, the fungal quorum sensing molecule farnesol referred to above 


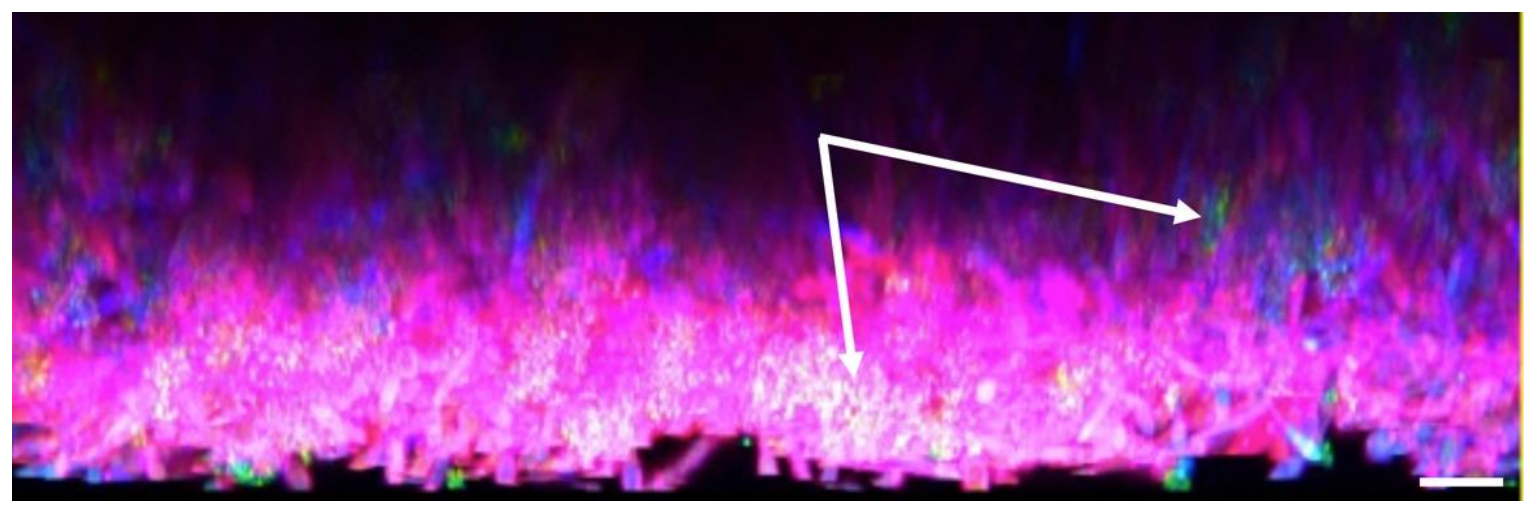

Figure 1-2. In vitro polymicrobial biofilm formation by $C$. albicans and S. aureus. The image demonstrates $S$. aureus (green/merged, white arrows) attached throughout the biofilm and along the hyphal filaments of $C$. albicans (blue). The extracellular matrix (red) largely encases a majority of the staphylococci. Methods: A polymicrobial biofilm was formed in vitro in RPMI-1640 medium with C. albicans (strain SC5314) and S. aureus (strain M2) using $1 \times 10^{6} \mathrm{CFU}$ of each microbe to inoculate a Permanox chamber slide for $24 \mathrm{~h}$ at $37^{\circ} \mathrm{C}$. Biofilms were washed with sterile saline to remove non-adherent cells, fixed in $4 \%$ formalin, and stained with a cocktail containing calcofluor white (50 $\mu \mathrm{g} / \mathrm{mL})$, Concanavalin A-Texas Red $(50 \mu \mathrm{g} / \mathrm{mL})$, and Syto9 $(1.67 \mu \mathrm{M})$. Images were captured using $405 \mathrm{~nm}, 488 \mathrm{~nm}$, and $565 \mathrm{~nm}$ lasers and DAPI, FITC, and Texas Red filter sets with a Zeis 510 confocal scanning laser microscope. Corresponding Z-stacks were constructed using packaged Zeis software depicting a side view. Scale bar represents 20 $\mu \mathrm{m}$. 


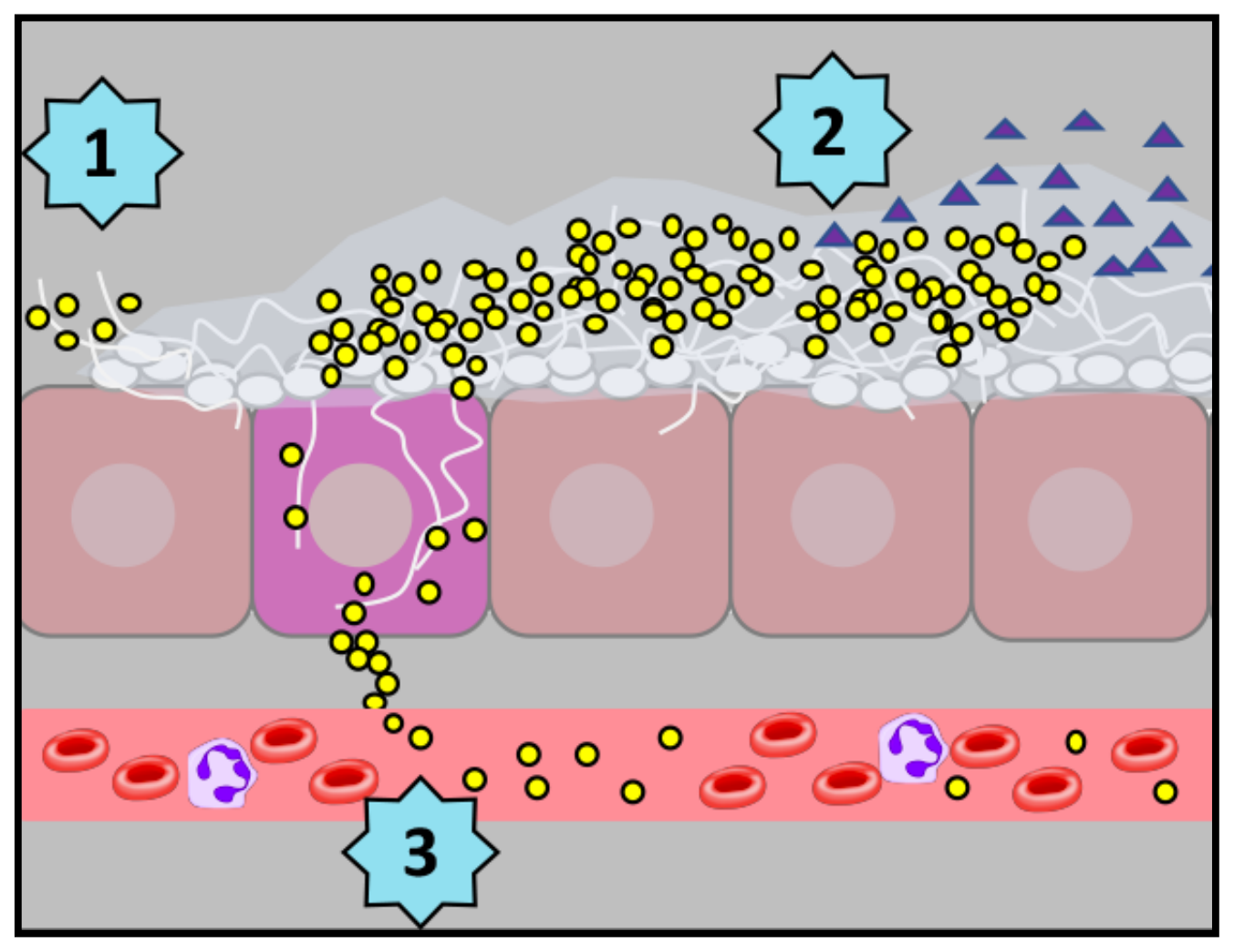

Figure 1-3. Schematic of C. albicans-S. aureus interactions.

(1) $S$. aureus preferentially attaches to the hyphal filaments of $C$. albicans via binding of the candidal adhesin Als3p. (2) Encasement of $S$. aureus in fungal biofilm matrix components (including $\beta$-1,3-glucan) impairs penetration of antibiotics (purple triangle) by sequestration of drug. The $C$. albicans QS molecule farnesol also upregulates drug efflux pumps in $S$. aureus to enhance tolerance to antibacterials. (3) $S$. aureus is able to gain access into subepithelial spaces by "hitchhiking" onto the invasive $C$. albicans hyphae. S. aureus may also then disseminate to distant sites (including the kidneys) following co-invasion via the bloodstream. 
was shown to activate staphylococcal drug efflux pumps, enhancing recalcitrance to several antibacterial drugs (49). Thus, it is clear that co-culture of these organisms can drastically alter phenotypic outcome with respect to drug tolerance and biofilm architecture (Figure 1-3).

\section{Enhanced Pathogenicity in the Oral Cavity and at a Distance}

A major virulence factor of $C$. albicans is the ability to switch from yeast to hyphae. The yeast form is suited for dissemination and initial seeding during infections, while the hyphal form is crucial for tissue penetration and immune evasion and is associated with upregulation of other virulence factors. One of these virulence factors is the production of surface adhesins. Peters et al. demonstrated that $S$. aureus binds to $C$. albicans hyphae through interaction with the candidal adhesin Als3p (50). Confocal fluorescence microscopy of $C$. albicans $-S$. aureus biofilms qualitatively showed decreased association of $S$. aureus to $C$. albicans hyphae lacking Als $3 p$ as compared to wild-type. The strength of this interaction was quantified by measuring the adhesion forces between $C$. albicans and $S$. aureus by atomic force microscopy, confirming weaker binding when Als3p was genetically deleted (50). The importance of Als3p binding by $S$. aureus during infection was demonstrated in an oral model of polymicrobial infection (51). When the oral cavity was infected with $C$. albicans and $S$. aureus, immunocompromised mice developed systemic infections with high microbial burdens in the kidneys and elevated mortality. Mono-infected mice (C. albicans or $S$. aureus alone) as well as mice infected with $S$. aureus and $C$. albicans als $3 \Delta / \Delta$ did not develop systemic infection (51). Similar results were found when $S$. aureus was coinoculated with the hypha-defective efg $1 \Delta / \Delta / \operatorname{cph} 1 \Delta / \Delta$ mutant of $C$. albicans (52). Thus, both hyphae and Als $3 p$ are required for $S$. aureus to disseminate from the oral cavity. The staphylococcal receptors required for robust binding to Als3p are likely multifactorial, although $S$. aureus mutants defective for fibronectin binding protein B $(\Delta f n b)$, staphylococcal surface protein $\mathrm{F}(\Delta s a s F)$, and autolysin $(\Delta a t l)$ demonstrated reduced capacity to bind hyphae in vitro (51). Cumulatively, these results led to development of a hypothetical model in which $S$. aureus could "hitchhike" onto the invasive filaments of C. albicans, gain access to submucosal tissue, and disseminate to distant sites

(Figure 1-3). Fluorescence in-situ hybridization images of tongue tissue from co-infected mice supported this hypothesis, as hyphae embedded deep into the epithelium were found surrounded by attached staphylococci (51).

C. albicans and $S$. aureus are both implicated in denture stomatitis, where the oral mucosa is inflamed and lesions are formed (37). A study by Baena-Monroy et al. examined saliva and culture swabs of denture surfaces from over 100 subjects fitted with dentures (53). Using culture-based techniques, they found that $C$. albicans and S. aureus could be recovered from the oral mucosa and the denture surfaces of both denture stomatitis patients and healthy controls. However, increased levels of C. albicans was recovered from the denture surface, while $S$. aureus was found predominantly in the oral mucosa of denture stomatitis cases. These results suggest that $C$. albicans may facilitate colonization of $S$. aureus during denture stomatitis and enable staphylococcal 
superinfection via a mechanism that could be explained by the aforementioned coinvasion hypothesis.

\section{Polymicrobial Intra-Abdominal Infection and Lethal Synergism}

Intra-abdominal infections (IAI) are a collection of a spectrum of diseases characterized by microbial infection within the abdominal cavity and resulting inflammation of the peritoneum. The majority of these infections are caused by a breach of the gastrointestinal tract epithelium, facilitating the invasion of microbes (54). IAI are the second most common cause of sepsis in intensive care unit (ICU) patients and typically have a high mortality rate (55). Polymicrobial IAI are correlated with a more severe disease state and higher rate of mortality, specifically when a fungal pathogen is involved, with mortality reaching $80 \%$ (56). This is in contrast to IAI caused by bacteria only, which display associated mortality rates of up to $30 \%$ (57). C. albicans and $S$. aureus are among the top most commonly isolated organisms during IAI (56).

Dissemination of microbes from the peritoneal cavity leads to systemic infection and can progress to sepsis. Sepsis is caused by the dysregulation of the immune system in response to infection (often hyper-inflammatory, followed by anergy) and is associated with severe organ damage and failure with a rapid onset of mortality (58).

Early studies conducted by Carlson described a synergistic effect on mortality in a mouse model of $C$. albicans-S. aureus polymicrobial IAI (59). The LD50 of the $C$. albicans strain was $2.9 \times 10^{8} \mathrm{CFU}$ (colony-forming unit); the LD50 of the $S$. aureus strain (2460, isolated from a patient with toxic shock syndrome) was determined to be $8 \times 10^{8}$ CFU. Outbred CD-1 mice were infected intraperitoneally (i.p.) with C. albicans $\left(7 \times 10^{6}\right.$ $\mathrm{CFU}), S$. aureus $\left(8 \times 10^{7} \mathrm{CFU}\right)$, or $C$. albicans $+S$. aureus at these same doses in saline. Results indicated that monomicrobial infection of either $C$. albicans or $S$. aureus was nonlethal, whereas dual infection with these sub-lethal doses of each organism caused nearly $100 \%$ mortality within three days post infection (d p.i.). Further experiments indicated that the ideal ratio of $S$. aureus: $C$. albicans to display synergistic lethality was approximately 10:1. Additionally, heat-inactivation of either organism eliminated this apparent synergism (59). These experiments helped develop initial concepts of synergistic lethality, whereby two microbes interact in a way that augments the virulence of one or both organisms, leading to enhanced morbidity and/or mortality.

Subsequent studies by Carlson evaluated the interaction between C. albicans and additional pathogenic bacteria, including Serratia marcesans and Enterococcus faecalis (60). Interestingly, C. albicans was able to enhance virulence with all bacterial species tested, again using sub-lethal doses of both fungi and bacteria. Analysis of the blood, the pancreas, the kidney, and the spleen demonstrated that E. faecalis or S. marcesans burdens were nearly identical in the respective tissues, regardless of the initial dose when given along with a standard dose of $C$. albicans. It was also noted that the bacterial burden in mice infected with bacteria alone was undetectable, whereas $C$. albicans colonization was unaltered by the presence or the absence of bacteria. Thus, a major conclusion from this work was that, although $C$. albicans amplifies the virulence of other 
microbes, this synergism does not seem to be mutual, as the bacteria tested did not appear to affect the colonization or the virulence of $C$. albicans during polymicrobial IAI (60).

Carlson further defined the infectious relationship between $C$. albicans and $S$. aureus in murine polymicrobial IAI. Robust synergistic lethality was only observed when both pathogens were given i.p. (61). Interestingly, when $C$. albicans was given i.p. and $S$. aureus was inoculated subcutaneously (s.c.), lethality was observed in $30 \%$ of animals, and mixed infection was established only at the site of fungal inoculation. This suggests that $S$. aureus is migratory during infection and that $C$. albicans is needed for staphylococcal colonization to persist, perhaps due to some yet identified protective effect provided by the fungi (61). Despite these important fundamental findings, the mechanism of synergistic lethality between $C$. albicans and $S$. aureus in polymicrobial intra-abdominal infection was left largely undefined until recently.

\section{Mechanisms of Synergistic Lethality: A Role for Staphylococcal Toxins}

Decades following the groundwork laid by Carlson, a new study emerged providing insight into the pathogenesis of $C$. albicans-S. aureus IAI (62). Similar to previous findings, intra-abdominal co-infection with $C$. albicans and $S$. aureus led to synergistic lethality with mice succumbing to infection by approximately $48 \mathrm{~h}$ p.i. Attention to the host response revealed significantly higher levels of neutrophils recruited to the peritoneal cavity during co-infection along with synergistic increases in cytokines Interleukin-6 (IL-6), Granulocyte-Colony Stimulating Factor (G-CSF), Keratinocyte Chemoattractant (KC), Monocyte Chemoattractant Protein-1 (MCP-1), and Macrophage Inflammatory Protein 1- $\alpha$ (MIP-1 $\alpha$ ) in the spleen and the kidneys, indicating robust inflammation. Interestingly, this study also revealed synergistic increases of the eicosanoid Prostaglandin $\mathrm{E}_{2}\left(\mathrm{PGE}_{2}\right)$ in the peritoneal lavage fluid. Prophylactic reduction of $\mathrm{PGE}_{2}$ using the cyclooxygenase inhibitor indomethacin significantly protected mice from co-infection and lowered inflammatory markers. Importantly, staphylococcal toxins, including $\alpha$ - and $\delta$-toxin, have been shown to activate phospholipase A2 signaling, leading to increased generation of prostaglandins $(63,64)$.

In order to unravel the mechanism of interaction between these two important pathogens, one must consider the virulence determinants of both organisms as well as the host response in driving the synergistic lethality. For $C$. albicans, this includes the morphological switch from yeast to hyphae and adhesive factors. Interestingly, in contrast to the oral co-infection model, Als3p binding was found to have no influence on synergistic mortality during $C$. albicans $-S$. aureus polymicrobial intra-abdominal infection (65). This finding was indirectly supported by Nash et al. in their investigation into the role of morphogenesis during $C$. albicans-S. aureus polymicrobial infection. This study showed that a yeast-locked $C$. albicans strain had no defect in promoting lethal synergism with $S$. aureus during polymicrobial IAI as compared to co-infection with a wild-type strain (66). Additionally, a hypha-"locked" C. albicans strain did not show enhanced mortality as compared to wild-type infection (66). Moreover, co-infection with a variety of non-albicans Candida species resulted in disparate infectious outcomes. 
Co-infection of S. aureus with Candida krusei (does not form hyphae) led to synergistic mortality similar to that of co-infection with $C$. albicans, while co-infection with Candida dubliniensis (close filamentous phylogenetic relative of $C$. albicans) was non-lethal (67). These data indicate that the morphology of $C$. albicans is not required for its ability to enhance $S$. aureus virulence during IAI. Interestingly, co-infection with the hyphadefective efg $1 \Delta / \Delta / \operatorname{cph} 1 \Delta / \Delta$ mutant failed to drive lethal synergism, indicating that expression of downstream target genes and not hyphal growth per se are important factors promoting infectious synergism (68).

As mentioned prior, $S$. aureus virulence is mainly due to toxin production and is intimately tied to QS. However, not all S. aureus strains have the same toxin profile, as major inter-strain heterogeneity with respect to genetic presence/absence and relative expression of toxin-producing genes is apparent $(69,70)$. Carlson used various $S$. aureus strains with different toxin profiles in her initial studies of C. albicans-S. aureus synergism, focusing on toxic-shock syndrome (TSS)-associated isolates and non-TSS disease-associated isolates (71). Unsurprisingly, the majority of TSS-associated isolates were positive for toxic shock syndrome toxin (TSST), while the non-TSS isolates were negative. She found that polymicrobial infection in mice with C. albicans and TSSassociated S. aureus strains led to $100 \%$ mortality within 2 d p.i., whereas non-TSSassociated strains caused $100 \%$ mortality much sooner, typically within $15 \mathrm{~h}$ p.i. Due to the lack of genetic tools and isogenic strains at the time, further characterization of specific toxins was not possible, thus it is unknown which other toxin(s) played a role in these results (71). Despite this, Carlson's work using spent culture supernatants from $S$. aureus during co-infection clearly hinted that staphylococcal exotoxins contribute to synergistic lethality (72).

\section{Lessons Learned, Yet Questions Remain}

While we have gained significant insight into the complex relationship existing between $C$. albicans and $S$. aureus, there is still much to learn regarding this fascinating microbial pair. Therefore, the purpose of this work was to answer several of the many remaining questions regarding the mechanism of synergistic lethality between $C$.

albicans and $S$. aureus: What are the specific factors, both fungal and bacterial, that contribute to lethality? How does the host respond to polymicrobial infection, and is this response a significant cause of morbidity and mortality? What exactly is driving lethality: microbial growth and dissemination, organ damage, sepsis, hyperinflammatory responses? How can we prevent, detect, and effectively treat these types of infections? As sequencing technologies become less expensive and more sensitive, it will be imperative to understand how microbiome, metagenome, and immune system shape these interactions within the human host, so that optimal and targeted therapies can be devised. Along with others, these case studies regarding C. albicans-S. aureus interactions have allowed the microbiology field to peer beyond the monomicrobial paradigm that Koch proposed a century before and have helped usher in a new frontier. If this much information has been gleaned from a single fungal-bacterial pairing, exciting discoveries on the horizon regarding additional microbial interactions are virtually endless. 


\section{CHAPTER 2. CANDIDA ALBICANS AUGMENTS STAPHYLOCOCCUS AUREUS VIRULENCE BY ENGAGING THE STAPHYLOCOCCAL AGR QUORUM SENSING SYSTEM*}

\section{Introduction}

The pathogenic fungus Candida albicans and ubiquitous bacterial pathogen methicillin-resistant Staphylococcus aureus (MRSA) remain serious clinical threats (73, 74). Together, these microorganisms rank among the most prevalent causes of nosocomial sepsis and catheter-related bloodstream infections, and recent reports have identified their coisolation with increasing frequency $(75,76)$. While polymicrobial infection is often associated with poor patient prognosis, studies designed to mechanistically evaluate microbial community composition and fungal-bacterial interactions in the context of host immunity are still in their infancy (77).

Comprehensive epidemiological data on $S$. aureus has identified several distinct clades, each characterized by unique disease pathology and virulence factors. Among these are USA200 and USA300 strains, which are commonly referred to as hospital acquired (HA) or community acquired (CA) MRSA, respectively. Generally, HA-MRSA strains are robust formers of staphylococcal biofilm and demonstrate wider antimicrobial resistance profiles, and some clades (e.g. CC30) exhibit lower levels of secreted bacterial toxins (78). These strains are often associated with orthopedic and medical device-related infections. Conversely, CA-MRSA strains secrete comparatively higher levels of bacterial toxin, including Panton-Valentine Leukocidin (PVL) and the pore-forming cytolytic $\alpha$-toxin (73). These strains have been associated with skin infection outbreaks (e.g. in prisons, care facilities, locker rooms), and it is believed that high levels of toxin and other secreted factors allow for efficient skin-skin and skin-fomite transfer. Toxin expression in $S$. aureus has been linked with multiple bacterial sensory regulators, but perhaps the best studied is the $a g r$ (accessory gene regulator) quorum sensing system (79).

The agr system is the product of the RNAII transcript, consisting of an operon composed of four genes (agrA,B,C,D) (79). AgrD is the signal peptide, which gets secreted across the bacterial membrane into the extracellular space and modified to its mature form, auto-inducing peptide 2 (AIP-2), via translocation through AgrB. AIP-2 is sensed by the cell surface-associated AgrC receptor that induces phosphorylation of AgrA. Activated AgrA can then bind again to the P2 promoter to increase RNAII transcription, completing a positive feedback cycle that is increased in a cell-density dependent fashion (i.e. quorum sensing). However, activated AgrA can also bind to the

\footnotetext{
*Reprinted from final submission with open access permission. Todd OA, Fidel PL, Jr., Harro JM, Hilliard JJ, Tkaczyk C, Sellman BR, Noverr MC, Peters BM. 2019. Candida albicans Augments Staphylococcus aureus Virulence by Engaging the Staphylococcal agr Quorum Sensing System. mBio 10.(65).
} 
P3 promoter to drive expression of the RNAIII transcript, which directly encodes for $\delta$ toxin and a post-transcriptional regulatory RNA. Expression of RNAIII decreases transcription of rot (repressor of toxin), thereby increasing exotoxin secretion (80). Both $\alpha$ - and $\delta$-toxin are well-characterized staphylococcal virulence factors, mediating a variety of pathological effects, including hemolytic activity, dermonecrosis, inflammasome activation, abscess formation, leukocyte oxidative burst, and reduced macrophage phagocytic killing (81-85). Toxin production by $S$. aureus is crucial for systemic disease, as high titers of antibody to staphylococcal exotoxins (including $\alpha$ toxin) directly correlates with improved survival rates during clinical $S$. aureus sepsis (86).

A series of studies by Carlson demonstrated that peritoneal coinoculation of mice with $C$. albicans and $S$. aureus resulted in synergistic increases in mortality, while mice inoculated with each of these microbes alone efficiently cleared the infection $(59,71,72)$. It was also observed that not all strains of $S$. aureus resulted in polymicrobial infectious synergism equally, with some decreasing the staphylococcal LD50 by as much as 70,000fold while others demonstrated only modest synergistic effects (2-3 fold) (71). It was proposed that expression of specific staphylococcal toxins governed increased mortality, namely $\alpha$-toxin and $\delta$-toxin. Unfortunately, lack of isogenic controls complicated data interpretation. Recently, our lab recapitulated these findings using a USA200 strain of $S$. aureus (NRS383) and identified that the host eicosanoid prostaglandin $\mathrm{E}_{2}\left(\mathrm{PGE}_{2}\right)$ is associated with disease severity and pharmacologic blockade of $\mathrm{PGE}_{2}$ synthesis and $\mathrm{PGE}_{2}$ receptors 1 and 3 dramatically improves survival rate $(62,66,87)$. Interestingly, staphylococcal exotoxins have been implicated in activation of phospholipase A2 and subsequent prostaglandin release $(63,88)$. Furthermore, synergistic effects on mortality and $\mathrm{PGE}_{2}$ generation during coinfection with $S$. aureus are independent of the capacity to undergo fungal morphogenesis (the major virulence attribute of $C$. albicans) and are not limited to $C$. albicans, as various other Candida species (including $C$. dubliniensis, $C$. tropicalis, and $C$. krusei) also enhance morbidity and mortality during intra-abdominal infection (IAI) $(66,67)$.

Regarding the potential link to toxin expression and synergistic lethality with $C$. albicans, the objective of this study was to identify whether staphylococcal toxins may be required for lethal co-infection and/or whether toxin expression is elevated during polymicrobial IAI. In support of this, we show strong in vitro and in vivo evidence that staphylococcal $\alpha$-toxin is necessary for robust infectious synergism and that $C$. albicans augments staphylococcal toxin production via engagement of the agr quorum sensing system. 


\section{Materials and Methods}

\section{Ethics Statement}

The animals used in this study were housed in AAALAC-approved facilities located at the University of Tennessee Health Sciences Center (UTHSC) in the Regional Biocontainment Laboratory. The UTHSC Animal Care and Use Committee, Laboratory Animal Care Unit (LACU) approved all animal usage and protocols (protocol \#18-060). Mice were given standard rodent chow and water ad libitum. Mice were monitored daily for signs of distress, including noticeable weight loss and lethargy. UTHSC LACU uses the Public Health Policy on Humane Care and Use of Laboratory Animals (PHS) and the Guide for the Care and Use of Laboratory Animals as a basis for establishing and maintaining an institutional program for activities involving animals. To ensure high standards for animal welfare, UTHSC LACU remains compliant with all applicable provisions of the Animal Welfare Act (AWAR), guidance from the Office of Laboratory Animal Welfare (OLAW), and the American Veterinary Medical Association Guidelines on Euthanasia.

\section{Strains and Growth Conditions}

C. albicans strain SC5314 (wild-type, reference isolate) was used for all experiments. The following $S$. aureus strains obtained from the Biodefense and Emerging Infectious (BEI) Research Resources repository were used: JE2 (WT, USA300 derived from strain LAC but cured of erythromycin resistance and cryptic plasmids), NE1532 $(\triangle a g r A)$, and NE1354 ( $\Delta h l a)(89)$. The S. aureus reporter strain harboring plasmid pDB22 encoding for erythromycin resistance and a P3 promoter fused to GFP mut2 was a kind gift from Dr. Pete Greenberg (University of Washington) (90). Using standard techniques, plasmid pDB22 was isolated from MN8-pDB22 and transformed into strain JE2 by electroporation to yield S. aureus(pDB22) (61). Using fluorescence microscopy, it was confirmed that $S$. aureus(pDB22) exhibited robust GFP expression during stationary growth, consistent with agr activation (Figure 2-1). All strains were maintained as $20 \%$ glycerol stocks and stored at $-80^{\circ} \mathrm{C}$.

Unless specifically noted otherwise, C. albicans was streaked onto Yeast Peptone Dextrose (YPD) agar. Isolated colonies were selected and inoculated into liquid YPD medium and grown overnight at $30^{\circ} \mathrm{C}$ with shaking at $200 \mathrm{rpm}$. S. aureus was streaked onto Trypticase Soy Agar (TSA) with antibiotic selection as required. Isolated colonies were selected and inoculated into liquid Trypticase Soy Broth (TSB) medium and grown overnight at $37^{\circ} \mathrm{C}$ with shaking at $200 \mathrm{rpm}$. The following day cultures were diluted 1:100 in fresh TSB and returned to the shaking $37^{\circ} \mathrm{C}$ incubator for $3 \mathrm{~h}$ until cultures reached the logarithmic phase of growth. 


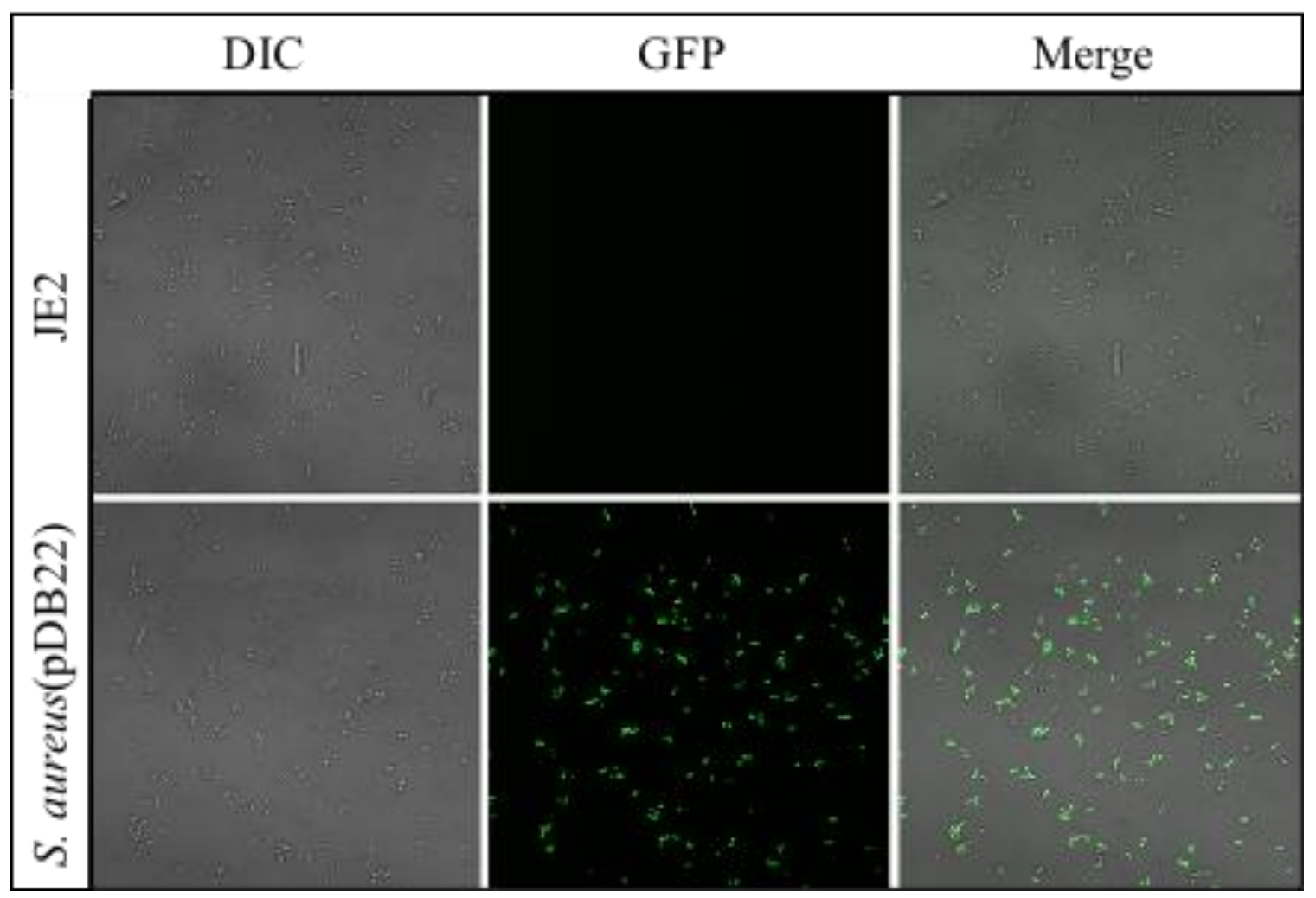

Figure 2-1. S. aureus(pDB22) demonstrates GFP expression in a cell density dependent manner, consistent with predicted agr/P3 promoter activity.

Reporter plasmid pDB22 was isolated from S. aureus strain MN8 and transformed into strain JE2 to yield strain $S$. aureus(pDB22). Specificity of GFP signal was assessed by inoculating both strains into TSB-g, incubating at $37^{\circ} \mathrm{C}$ for $16 \mathrm{~h}$, and imaging by fluorescence microscopy using a DIC/GFP filter set. 


\section{Construction of an hla-Complemented Strain}

In order to complement $\alpha$-toxin expression, primers (restriction enzyme sites indicated by underlined text) hlaF-HindIII (5'-GTAAAGCTTCATACGATACTTTTTC GTTATCTATTAG) and hlaR-BamHI (5'-CGGGGATCCCAGTATAAAAATTAG CCGAAAAACATCATTTCTG) were used to PCR amplify genomic DNA isolated from strain JE2 to generate a fragment containing the entire hla open reading frame, $\sim 500 \mathrm{bp}$ of the 5' untranslated region (UTR), and 100 bp 3'UTR. Vector pSK5630 (a low copy plasmid, 5 per cell) and the hla PCR product were digested with HindIII and BamHI and ligated to yield plasmid pSK-hla. The dcm-deficient Escherichia coli strain IM08B was made chemically competent and used as a propagation host for pSK-hla after standard heat-shock transformation and selection on Luria-Bertani (LB) agar containing ampicillin $(50 \mu \mathrm{g} / \mathrm{mL})$ to yield strain IM08B-phla (91). pSK-hla was isolated from selective overnight cultures of IM08B-phla using a miniprep procedure according to manufacturer's protocol (GeneJet, ThermoFisher) and verified by restriction digest with HindIII and BamHI, followed by Sanger sequencing (UTHSC, Molecular Resource Center). Using a vendor optimized protocol for staphylococcal electroporation (GenePulser Xcell, Bio-rad), pSK-hla was transformed directly into strain NE1354 ( $\Delta$ hla background) and plated on Brain Heart Infusion agar containing chloramphenicol (10 $\mu \mathrm{g} / \mathrm{mL}$ ) to yield strain $\Delta$ hla-phla. Individual colonies were replica plated onto TSA agar containing 5\% sheep's blood and chloramphenicol $(10 \mu \mathrm{g} / \mathrm{mL})$ to screen for those with a hemolytic phenotype. Plasmids were isolated from hemolysis-positive colonies by miniprep procedure using a $S$. aureus-specific manufacturer-supplied protocol (Qiaprep, Qiagen) and verified by PCR amplification using primers pSK5630-F (5'-ACGATGCG ATTGGGATATATCAACG) and hlaDETR (5'-GTGTTGTTGTTACTGAGCTGACT ATACG).

\section{Murine Model of IAI}

Intraperitoneal (i.p.) inoculations were conducted as described previously $(62,66$, 67). In most experiments, groups $(n=4)$ of six-week old Swiss Webster mice were injected i.p. using a 27-gauge $1 / 2$ " needle with $1.75 \times 10^{7} \mathrm{CFU}$ of C. albicans, $8 \times 10^{7}$ CFU of $S$. aureus, or $1.75 \times 10^{7}$ and $8 \times 10^{7} \mathrm{CFU}$ of the respective microbe simultaneously. Inocula were prepared in a final volume of $0.2 \mathrm{~mL}$ pyrogen-free phosphate-buffered saline (PBS). After inoculation, mice were observed up to $10 \mathrm{~d}$ p.i. for morbidity (hunched posture, inactivity, ruffled fur) and mortality. Mice that exhibited severe morbidity were humanely sacrificed and tallied as a lethal outcome. In some experiments, mice were sacrificed $8 \mathrm{~h}$ p.i. prior to severe morbidity. Peritoneal cavities were lavaged by injection of $2 \mathrm{~mL}$ of sterile PBS containing 1X protease inhibitors (cOmplete, Roche) followed by gentle massaging of the peritoneal cavity. Peritoneal lavage fluid was then removed using a pipette inserted into a small incision in the abdominal cavity. Animal experiments were repeated in duplicate and results combined. 


\section{Passive Immunization with an Anti- $\alpha$-Toxin Monoclonal Antibody}

Groups of mice $(n=4)$ were inoculated i.p. with $200 \mu$ of the $\alpha$-toxin specific IgG1 neutralizing antibody MEDI4893* $(15 \mathrm{mg} / \mathrm{kg}, 45 \mathrm{mg} / \mathrm{kg}$ ) or human IgG1 isotype control R347 (45 mg/kg) prepared in sterile PBS $24 \mathrm{~h}$ prior to co-infection with $C$. albicans and S. aureus. Mice were monitored for up to $10 \mathrm{~d}$ p.i. for morbidity and mortality. Animal experiments were repeated in duplicate and results combined.

\section{Intraperitoneal Delivery of $\alpha$-Toxin}

Groups of mice $(n=4)$ were inoculated i.p. with $0.2,0.5,0.75,1$, or $5 \mu \mathrm{g}$ of purified native $\alpha$-toxin in $0.1 \mathrm{~mL}$ of PBS alone or sequentially with $1.75 \times 10^{7} \mathrm{CFU}$ of $C$. albicans in $0.1 \mathrm{~mL}$ PBS (84). Mice inoculated with $\alpha$-toxin only received a sham injection of $0.1 \mathrm{~mL}$ PBS. Mice were monitored for morbidity and mortality up to $10 \mathrm{~d}$ p.i. Data is representative of at least 2 independent repeats.

\section{CFU Analysis}

Microbial burdens were enumerated by serial dilution plating of peritoneal lavage fluid and culture media onto YPD containing $20 \mu \mathrm{g} / \mathrm{mL}$ ampicillin and $2 \mu \mathrm{g} / \mathrm{mL}$ vancomycin (for $C$. albicans enumeration) and TSA containing $20 \mu \mathrm{g} / \mathrm{mL}$ ampicillin and $2.5 \mu \mathrm{g} / \mathrm{mL}$ amphotericin B (for $S$. aureus enumeration) via the drop-plate method (92). Plates were incubated overnight at $37^{\circ} \mathrm{C}$, and the microbial burden was enumerated and expressed as CFU per $\mathrm{mL}$. CFU values are representative of at least 2 independent repeats $(n=4$ mice per group) and represented as the median $( \pm S D)$.

\section{Prostaglandin Quantitation}

Recovered lavage fluid was centrifuged at $500 \times \mathrm{xg}$ for $5 \mathrm{~min}$, and the supernatant was transferred to clean microfuge tubes. $\mathrm{PGE}_{2}$ is rapidly converted to its 13,14 -dihydro15-keto metabolite in vivo, so the colorimetric Prostaglandin E Metabolite competitive enzyme immunoassay (EIA) was used to measure $\mathrm{PGE}_{2}$ as a function of its breakdown product, according to the manufacturer's directions (Cayman Chemicals, Ann Arbor, MI). This assay is highly sensitive and detects as little as $2 \mathrm{pg} / \mathrm{mL}$ of PGE metabolites.

\section{S. aureus $\alpha$-Toxin Quantitation by ELISA}

Wells of a polystyrene 96-well microtiter plate were coated with $50 \mu \mathrm{l}$ of 0.1 $\mu \mathrm{g} / \mathrm{mL}$ anti- $\alpha$-toxin antibody MEDI $4893 *$ (diluted in coating buffer $(0.2 \mathrm{M}$ carbonate/bicarbonate buffer)) and incubated overnight at $4^{\circ} \mathrm{C}$. Plates were washed sequentially with PBS-Tween 20 (PBS-T) and blocked with SuperBlock (Pierce) for $1 \mathrm{~h}$ at room temperature. After washing with PBS-T, $50 \mu$ of diluted peritoneal lavage fluid 
or diluted filter-sterilized culture supernatants in PBS was added. Additionally, serial dilutions of native $\alpha$-toxin were included as the standard curve. The plate was then incubated for $1 \mathrm{~h}$ at room temperature with shaking $(600 \mathrm{rpm})$. After washing with PBS$\mathrm{T}, 50 \mu \mathrm{l}$ of affinity-purified rabbit polyclonal anti- $\alpha$-toxin antibody $(2 \mu \mathrm{g} / \mathrm{mL})$ was added and incubated as described above. After washing, $50 \mu \mathrm{l}$ of a 1:10,000 dilution of Affinipure horseradish peroxidase (HRP)-coupled goat anti-rabbit IgG detection antibody (Jackson Immuno Research) was added and incubated for $1 \mathrm{~h}$. Plates were washed extensively with PBS-T, and then $100 \mu \mathrm{l}$ of 3,3',5,5'-Tetramethylbenzidine (TMB) substrate was added for $10 \mathrm{~min}$, followed immediately by $100 \mu$ of ELISA stop solution $\left(0.2 \mathrm{M} \mathrm{H}_{2} \mathrm{SO}_{4}\right)$ was added. Wells were read at $450 \mathrm{~nm}$ using a plate reader (Synergy, Biotek), and experimental values were extrapolated to the standard curve. Culture supernatants and peritoneal lavage fluid generated from the $\Delta h l a$ strain were blank subtracted to account for any potential nonspecific antibody binding to shed protein A. Experiments were repeated in duplicate ( $\mathrm{n}=4$ mice per group), and data were combined and expressed as the mean \pm SEM.

\section{Agar Plate Assay for Toxin Activity}

Colonies of $S$. aureus (JE2, $\Delta a g r A, \Delta h l a)$ and $C$. albicans were grown overnight as described above, and cell densities adjusted to $1 \times 10^{7} \mathrm{CFU} / \mathrm{mL}$ by counting on a hemocytometer. A 1:100 dilution $(50 \mu \mathrm{l})$ was made into $5 \mathrm{~mL}$ of $0.6 \mathrm{X}$ TSB containing $0.2 \%$ glucose (TSB-g) (a medium previously shown to support good growth of both $C$. albicans and $S$. aureus) to establish the following groups: $S$. aureus alone, C. albicans alone, or $C$. albicans and $S$. aureus $(93,94)$. Monomicrobial cultures received an additional $50 \mu \mathrm{l}$ of sterile PBS. Tubes were placed in a $37^{\circ} \mathrm{C}$ incubator with shaking at $200 \mathrm{rpm}$. Aliquots were removed at several time points, centrifuged at $5000 \mathrm{rpm}$ to remove cellular debris, and passed through a $0.2 \mu \mathrm{m}$ syringe filter. Wells were aseptically formed in TSA agar blood containing 5\% sheep's blood using a cut sterile pipette tip. Sterile culture supernatant $(20 \mu \mathrm{l})$ was added to each well and the plate incubated at $37^{\circ} \mathrm{C}$ for up to $24 \mathrm{~h}$. Plates were imaged using a digital scanner and are representative of at least 3 independent repeats.

\section{RBC Hemolytic Assay}

An aliquot $(1 \mathrm{~mL})$ of rabbit blood (Lampire Biologicals) was centrifuged at $1000 x g$ for 5 min to pellet RBCs. Packed red cells were washed $2 X$ further by centrifugation, resuspended in $1 \mathrm{~mL}$, and a $4 \% \mathrm{v} / \mathrm{v}$ dilution of RBCs made. Isolated cellfree supernatants (as described above) were serially diluted in sterile TSB-g and $200 \mu \mathrm{l}$ added to wells of a microtiter plate. To this, $50 \mu \mathrm{l}$ of RBCs were added and plate incubated at $37^{\circ} \mathrm{C}$ for $1 \mathrm{~h}$ with gentle shaking. Plates were centrifuged at $1000 \mathrm{xg}$ for 5 min to pellet unlysed RBCs, supernatants transferred to a fresh microtiter plate, and the OD $405 \mathrm{~nm}$ collected as measure of hemoglobin release. Positive (1\% Triton-X 100) and negative (sterile TSB-g) controls were included. Experiments were repeated in triplicate, values normalized as \% of positive control, and expressed as the mean \pm SEM. 


\section{agrA-GFP Reporter Assay}

Culture setup was similar to the agar plate assay described above, except that the S. aureus(pDB22) strain was also included and $10 \mu \mathrm{g} / \mathrm{mL}$ of erythromycin was included for plasmid maintenance. Aliquots of cultures $(100 \mu \mathrm{l})$ were removed at various time points post-inoculation in TSB-g and placed into wells of a black microtiter plate in triplicate. Fluorescence (488 nm excitation, $515 \mathrm{~nm}$ emission) was captured on a plate reader (Synergy, Biotek). Experiments were repeated in triplicate, and results expressed as mean arbitrary fluorescence units (AFUs) \pm SEM.

\section{Fluorescence Microscopy}

Aliquots of cells from the reporter assay were stained with the fluorescent DNA stain Syto62 as per manufacturers protocol (Invitrogen) and placed onto cover-slipped glass slides. Images were captured on an Olympus FV1000 confocal microscope using GFP and Texas Red filter sets. Samples of peritoneal lavage fluid were placed onto glass slides and imaged using DIC and GFP filter sets. Images are representative of at least 3 independent repeats.

\section{RNA Isolation for qPCR}

Cultures were setup as described for the agar plate assay. RNA from $S$. aureus was selectively isolated as described, with some modifications (95). Briefly, cells were removed from the incubator and an equal volume of ice-cold 50\% Acetone $/ 50 \%$ ethanol was added to prevent RNA degradation. Cells were centrifuged $2 \mathrm{X}$ at $4000 \mathrm{rpm}$ and resuspended in $1 \mathrm{~mL}$ of DEPC-treated water. Cells were transferred to eppendorf tubes, centrifuged, and $5 \mu \mathrm{l}$ of lysostaphin added $(10 \mathrm{mg} / \mathrm{mL})$. The pellet was vortexed vigorously, resuspended in $200 \mu \mathrm{l}$ of DEPC water, and incubated in a $37^{\circ} \mathrm{C}$ water bath for $45 \mathrm{~min}$. To this, $2.5 \mu \mathrm{l}$ of proteinase K (Qiagen) was added and incubated for an additional $15 \mathrm{~min}$. After incubation, 0.2 volumes of $10 \%$ SDS was added, followed by an equal volume of $\mathrm{pH} 4$ 125:24:1 phenol:chloroform:isoamyl alcohol, and a small amount of $0.1 \mathrm{~mm}$ zirconia beads. Tubes were vortexed vigorously for $2 \mathrm{~min}$, followed by $1 \mathrm{~min}$ incubation on ice; this was repeated $3 \mathrm{X}$ total. RNA was extracted by the hot phenol:chloroform method $(5: 1, \mathrm{pH} 4)$, precipitated with $3 \mathrm{M}$ sodium acetate, and washed with $70 \%$ ethanol. The pellet was air-dried and resuspended in $25 \mu \mathrm{DEPC}$ water. Concentrations were assessed spectrophotometrically (A260/A280 ratio) and RNA integrity verified by running $\sim 1 \mu \mathrm{g}$ of RNA on a $1.4 \%$ TBE agarose gel. Analysis of $16 \mathrm{~S}$ and $23 \mathrm{~S}$ ribosomal (but lack of 18S and 28S) RNA bands confirmed that staphylococcal RNA from polymicrobial cultures was selectively extracted using this procedure. 


\section{qPCR for Staphylococcal Genes}

Trace amounts of contaminating DNA were removed from RNA samples $(1 \mu \mathrm{g})$ by treatment with RNase-free DNase I per the manufacturer's protocol (Thermo Fisher). RNA was reverse transcribed using the Revertaid first-strand kit and primed with random hexamers per the manufacturer's protocol (Thermo Fisher). cDNA (100 ng) was amplified using gene-specific primers (Sigma) and the Maxima Sybr Green 2X PCR mastermix per the manufacturer's protocol (Thermo Fisher). Amplification and fluorescence measurement were conducted using the 7500 Real Time PCR System platform (Applied Biosystems). Expression levels of target genes in polymicrobial cultures were compared to those in monomicrobial cultures and normalized to staphylococcal reference gene gyrB using the $\Delta \Delta \mathrm{C}_{\mathrm{T}}$ method as described previously (96). RNA was extracted from triplicate experiments, $\Delta \Delta \mathrm{C}_{\mathrm{T}}$ values calculated, averaged, and expressed as the mean $\pm \mathrm{SD}$.

\section{Western Blotting for Staphylococcal Proteins}

Cell-free supernatants were separated into ethanol-soluble and insoluble fractions by treatment with $80 \%$ final $\mathrm{v} / \mathrm{v}$ ice-cold ethanol for $1 \mathrm{~h}$ at $-80^{\circ} \mathrm{C}$. Samples were centrifuged at $3000 \mathrm{xg}$ for $15 \mathrm{~min}$. The ethanol-soluble fraction was transferred to a clean tube and both fractions were placed in a N-EVAP nitrogen evaporator (Organomation) set at $75^{\circ} \mathrm{C}$ and $10 \mathrm{~mm}$ flow. Upon reaching near-dryness, samples were resuspended in $0.5 \mathrm{~mL}$ of ultrapure water, and total protein content assessed by the bicinchoninic (BCA) assay (Pierce). Equivalent volumes $(40 \mu \mathrm{l})$ of concentrated supernatants were diluted in 6X Laemli sample buffer, boiled, and separated by SDS-PAGE in duplicate. We chose not to normalize to total protein, as the polymicrobial culture would have elevated levels of protein (both $C$. albicans and S. aureus) and may bias interpretation when compared to monomicrobial cultures. Ethanol-soluble fractions were assessed using Tris-Tricine electrophoresis and insoluble fractions using a Tris-Glycine system. Equivalent protein load was confirmed by staining one set of gels with Biosafe-Coomassie, while the other gel was transferred to nitrocellulose membranes and blocked in 5\% powdered milk PBS$\mathrm{T}$ for antibody probing as follows. To detect $\alpha$-toxin, a 1:10,000 dilution of primary rabbit anti- $\alpha$-toxin (Sigma, S7531) and 1:50,000 dilution HRP-coupled anti-rabbit IgG antibodies were used. To detect $\delta$-toxin, 1:1,000 dilution of primary rabbit anti- $\delta$-toxin (LSBio, LS-C156026) and 1:50,000 dilution HRP-coupled anti-rabbit IgG antibodies were used. To detect protein A, a 1:2,000 dilution of mouse anti-protein A (Abcam, ab181627) and 1:50,000 of goat anti-mouse IgG (Fab specific) were used. Primary antibodies were incubated overnight at $4^{\circ} \mathrm{C}$ in blocking buffer with gentle rocking. Secondary antibodies were incubated for $1 \mathrm{~h}$ at room temperature with gentle rocking. Gels were washed at least $3 X$ for 5 min with PBS-T between incubation steps. Signal was detected using the SuperSignal Chemiluminescent Substrate kit (Pierce) as per manufacturer's protocol and exposure images captured on the ChemiDoc XRS system (Bio-rad). Densitometry of resulting bands was calculated using ImageJ software (NIH). Blots are representative of 3 independent repeats. 


\section{Statistical Analysis}

A two-tailed Mann Whitney test was used to compare CFU values between polymicrobial and monomicrobial groups. A two-tailed unpaired Student's T-test was used to compare AFU and RBC lysis values for polymicrobial vs. monomicrobial groups. A Wilcoxon log-rank test was used to determine significance of mortality plotted in Kaplan-Meier curves. Statistical analyses were performed using GraphPad Prism. Significance is denoted as follows: * $P<0.05$; **, $P<0.01$. All graphs were constructed using GraphPad Prism. Figures were composed using MS Powerpoint and rendered for publication with Adobe Photoshop.

\section{Results}

\section{A USA300 Strain Exhibits Robust Synergistic Lethality During Coinfection}

Previous observations by Carlson, et al. revealed that $S$. aureus strains capable of producing various exotoxins demonstrated strong infectious synergism with $C$. albicans during IAI (59). S. aureus strain JE2 (derived from the USA300 isolate LAC) is a well characterized and robust producer of secreted toxins with an intraperitoneal LD50 of $5 \mathrm{x}$ $10^{8} \mathrm{CFU}$ (97). Therefore, we administered a sub-lethal dose of $S$. aureus JE2 to observe potential synergistic effects during co-infection. Intraperitoneal (i.p.) inoculation of Swiss-Webster mice with $8 \times 10^{7} \mathrm{CFU}$ S. aureus (SA, yellow) led to some morbidity by 1 day post-inoculation (d p.i.) (slightly ruffled fur) but ultimately these mice cleared the infection with no mortality (Figure 2-2). Similarly, infection with $C$. albicans alone (CA, blue) led no observable morbidity and no mortality (Figure 2-2). However, co-infection at this same dose with these microbes was striking (CA+SA, green). Mice began exhibiting symptoms of morbidity around $12 \mathrm{~h}$ p.i. By approximately $16-20 \mathrm{~h}$ p.i. $80 \%$ of mice had succumbed to infection. Therefore, the rapid onset of morbidity and mortality in co-infected mice may be explained by the relatively high toxigenic activity of this $S$. aureus strain (among other strain-dependent differences). However, S. aureus JE2 was non-lethal during monomicrobial infection. Thus, we hypothesized that co-infection with C. albicans may actually augment toxin expression.

\section{The agr Quorum Sensing System Is Enhanced During Coculture}

The staphylococcal agr quorum sensing system is tightly linked with toxin regulation. As part of a two-component signal transduction system, upon its activation AgrA is phosphorylated and drives expression of RNAIII, the major toxin effector, by binding to the P3 promoter. We constructed an agrA reporter strain in JE2 based on plasmid pDB22 [S. aureus(pDB22)] with the P3 promoter fused to GFP to be used as a proxy of toxin production. During planktonic culture in TSB-g, GFP levels were absent at early time points, consistent with the fact that activation of the agr system is cell density dependent (Figure 2-3A). At $\sim 8 \mathrm{~h}$ p.i. reporter activities were noticeable in both 


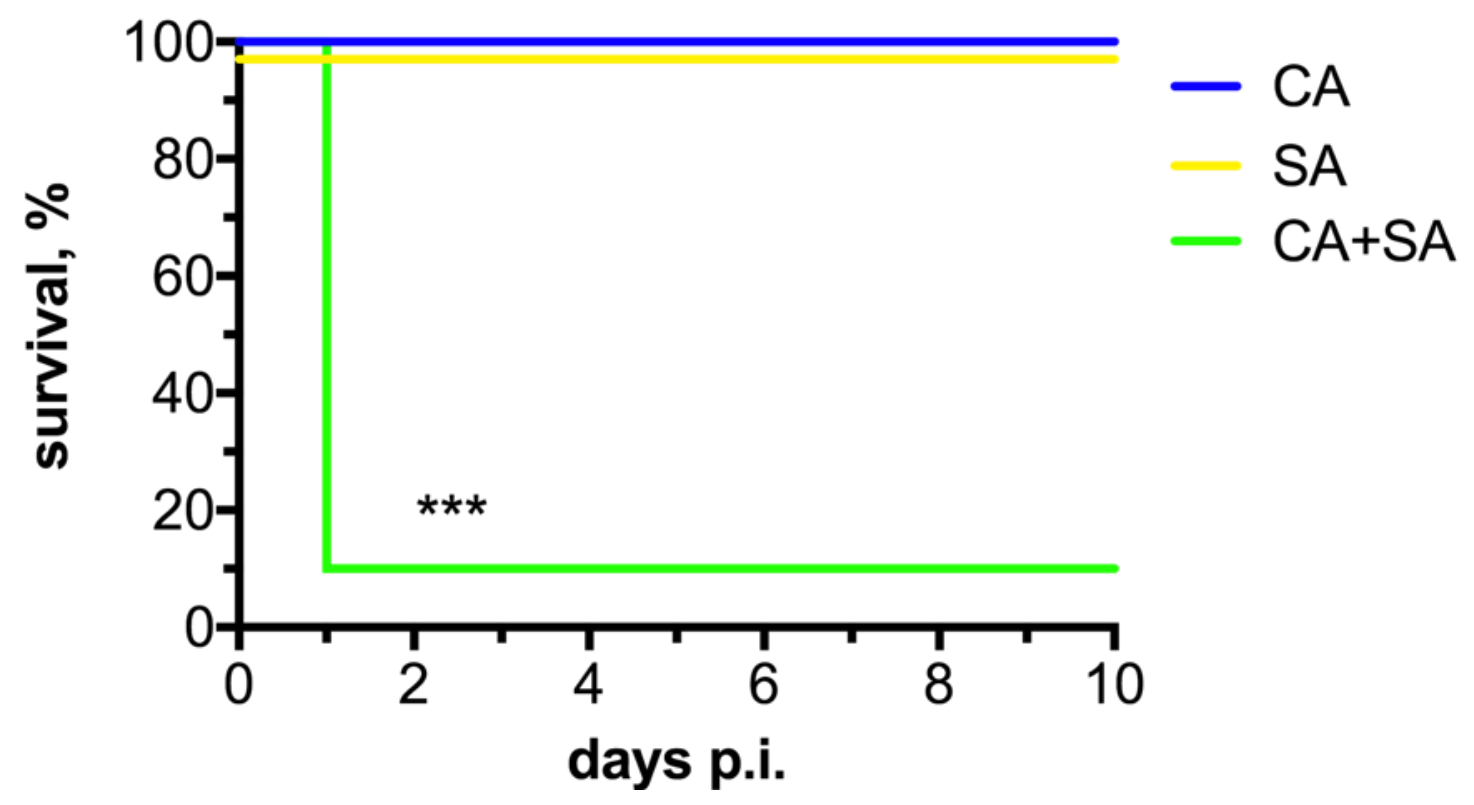

Figure 2-2. Co-infection with a USA300 strain leads to robust early synergistic mortality.

Mice ( $\mathrm{n}=8$ per group) were inoculated i.p. with $1.75 \times 10^{7} \mathrm{CFU} C$. albicans (CA, blue), $8 \times 10^{7} \mathrm{CFU}$ S. aureus (SA, yellow), or both pathogens simultaneously at these doses (CA+SA, green). Survival was monitored up to $10 \mathrm{~d}$ p.i. Data is derived from duplicate experiments of 4 mice per group and combined. Significance was assessed using a Wilcoxon rank sum test. ***, $P<0.001$. 
Figure 2-3. P3-reporter activity, a surrogate of staphylococcal agr activation, and red blood cell lysis is enhanced during co-culture with $C$. albicans.

(A) A P3-GFP reporter strain of $S$. aureus $[(S$. aureus(pDB22)] was incubated alone or with $C$. albicans over a $24 \mathrm{~h}$ time course. At each time interval, $100 \mu \mathrm{l}$ of culture was removed in triplicate, added to a 96-well plate, and fluorescence captured at $488 \mathrm{~nm} / 515$ nm. C. albicans only and $S$. aureus lacking pDB22 were also included as controls. Values represent the mean AFU of triplicate experiments \pm SEM. (B) Aliquots from reporter experiments were also visualized by fluorescence microscopy at $16 \mathrm{~h}$ by counter-staining cells with the DNA stain Syto62 and capturing GFP fluorescence with a GFP/Texas Red filter set. Images are representative of 3 independent repeats. (C) CFU levels $(C$. albicans, blue; $S$. aureus, yellow) of monomicrobial and polymicrobial cultures at $16 \mathrm{~h}$ growth were assessed by microbiological plating on selective media. Counts were assessed for significance using a Mann-Whitney U test. (D) Hemolytic activity was assessed from monomicrobial (C. albicans or S. aureus) and polymicrobial (C. albicans + $S$. aureus) cultures by placing $20 \mu \mathrm{l}$ of filtered culture supernatant into wells on a Sheep's blood agar plate. Images were captured after incubation at $37^{\circ} \mathrm{C}$ for $16 \mathrm{~h}$ and representative of 3 independent repeats. (E) agr activity was quantitatively assessed by the capacity of supernatants from monomicrobial (C. albicans, blue; S. aureus, yellow) or polymicrobial cultures (green) to lyse rabbit erythrocytes. Values are expressed as the mean of triplicate experiments \pm SEM. Significance of synergism was compared using a Student's t-test. *, $P<0.05$. 
A

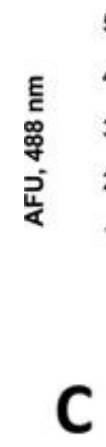

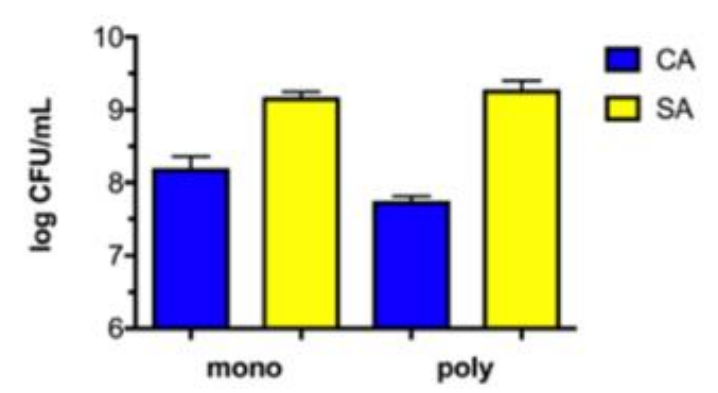

B

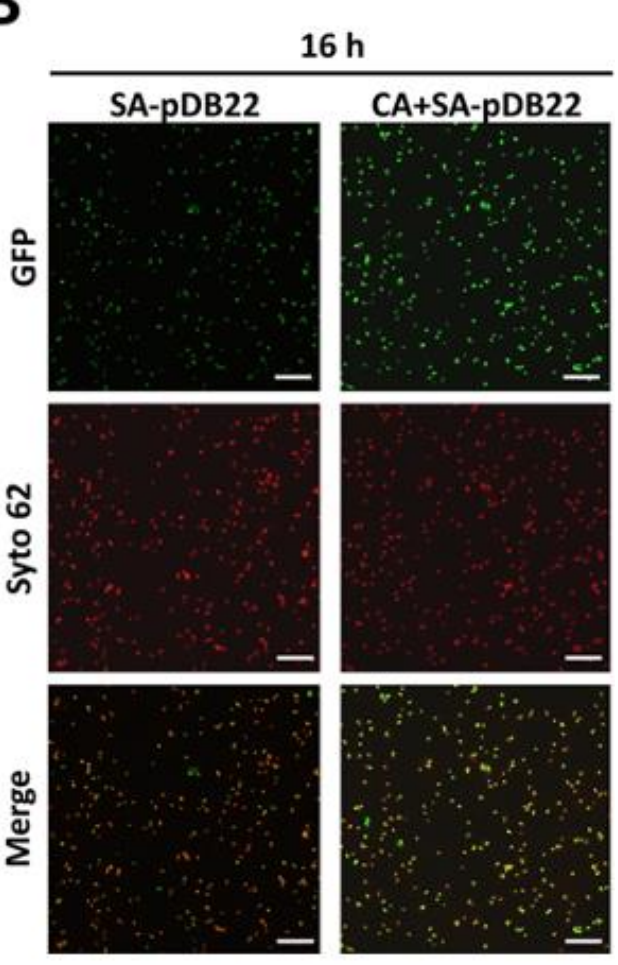

D

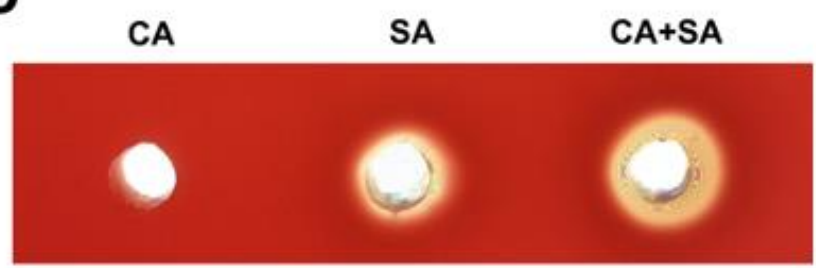

E

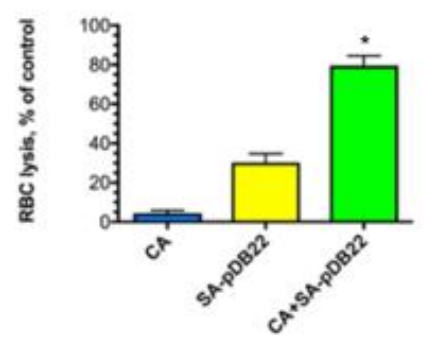


monomicrobial and polymicrobial cultures harboring $S$. aureus(pDB22). However, at 12 and $16 \mathrm{~h}$ reporter activity of polymicrobial cultures were significantly higher (as much as doubled) than during monomicrobial growth, indicating augmented activation of the major signaling pathway controlling toxin production (Figure 2-3A). To confirm these findings, aliquots from cultures were removed and imaged by fluorescence microscopy, revealing qualitatively enhanced GFP signal in polymicrobial as compared to monomicrobial cultures (Figure 2-3B). To address whether organism burden was drastically altered during polymicrobial growth at the $16 \mathrm{~h}$ time point, $C$. albicans, $S$. aureus or the two combined were grown in TSB-g and plated on selective microbiological media. Enumeration revealed nearly identical $C$. albicans and $S$. aureus CFU levels between both monomicrobial and polymicrobial cultures (Figure 2-3C). However, cell-free supernatants added to TSA-blood revealed strikingly higher levels of hemolysis from polymicrobial cultures, suggesting enhanced levels or activity of hemolytic toxins during growth with $C$. albicans, consistent with GFP reporter results. Importantly, cell-free supernatants derived from $C$. albicans cultures were devoid of hemolytic activity (Figure 2-3D). Augmented lysis of rabbit red blood cells (a cell type exquisitely sensitive to $\alpha$-toxin) from polymicrobial cultures further confirmed this phenotype quantitatively (Figure 2-3E) (98).

\section{agr-Regulated Genes and Corresponding Proteins Are Increased During Coculture}

In order to determine whether downstream agr-regulated genes were also being increased during polymicrobial growth, we assessed transcriptional responses of $S$. aureus or $C$. albicans in combination with $S$. aureus by qPCR. By rupturing cells with lysostaphin (an enzyme that breaks down staphylococcal cell walls) we were able to selectively isolate $S$. aureus RNA so that the amounts of recovered staphylococcal nucleic acid between monomicrobial and polymicrobial cultures could be made equivalent. qPCR analysis revealed increases in hld (encoding for $\delta$-toxin, 5.1-fold), hla ( $\alpha$-toxin, 80-fold), RNAII (agr operon, 2.5-fold), agrA (quorum sensing regulator, 7fold), and spa (surface protein A, -0.5-fold) (Figure 2-4A). As RNAII, hld, hla, and agrA itself are downstream targets of $a g r$ activation, these results provide further evidence of augmented $a g r$ activity during growth with $C$. albicans. The exception to this gene list was the down-regulation of spa. However, it has been well documented that surface protein A is negatively correlated with $a g r$ activation, and thus its down-regulation is in agreement with previous observations (99). Similar regulation of these quorum sensing effectors was also conserved at the protein level as evidenced by Western blotting of cellfree supernatants (Figure 2-4B). Phenol soluble modulins (of which $\delta$-toxin is a member) are small, ethanol soluble peptides that prove somewhat difficult to resolve by electrophoresis $(100,101)$. Therefore, we utilized an ethanol precipitation step and subsequent drying to fractionate supernatants to improve toxin recovery as described previously (100). Densitometric analysis demonstrated that $\alpha$-toxin $(37 \mathrm{kDa})$ was increased by 8.2 -fold, $\delta$-toxin $(3 \mathrm{kDa})$ was increased by 4.3 -fold, and Protein A (56 kDa) was decreased by 0.7 -fold, further explaining increased in vitro hemolytic activity. Protein expression levels were in line with transcriptional results, except for $\alpha$-toxin, 


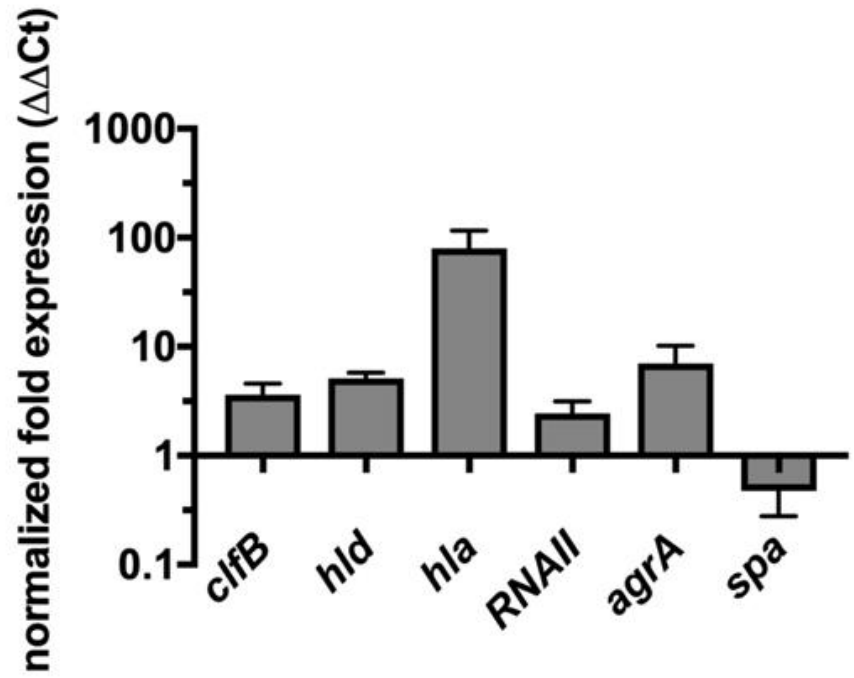

B

\begin{tabular}{|c|c|c|c|c|c|c|}
\hline Protein & Size (kD) & Marker & CA & SA & CA+SA & Fold-change \\
\hline a-toxin & 31 & $\infty$ & & $\longrightarrow$ & & +8.2 \\
\hline$\delta$-toxin & 5 & -1 & & & & +4.3 \\
\hline Protein A & 45 & m. & & & & -0.7 \\
\hline
\end{tabular}

Figure 2-4. agr-associated genes and staphylococcal toxins are increased during polymicrobial growth with $C$. albicans.

(A) Quantitative real-time PCR (qPCR) was performed to assess expression of staphylococcal quorum sensing related genes during monomicrobial vs. polymicrobial culture. Values were calculated using the $\Delta \Delta \mathrm{C}_{\mathrm{T}}$ method and normalized to reference gene $\operatorname{gyr} B$. Data is expressed as normalized fold-expression of polymicrobial vs.

monomicrobial cultures and the mean of 3 independent experiments. (B) Representative image $(\mathrm{n}=3)$ of Western blot performed on $40 \mu \mathrm{l}$ of cell-free supernatants derived from C. albicans (CA) only, S. aureus (SA) only, or polymicrobial (CA+SA) cultures for $\alpha$ toxin, $\delta$-toxin, and Protein A. 
which was lower than anticipated by qPCR and may indicate involvement of other mechanisms of post-transcriptional control (102).

\section{$\alpha$-Toxin Is Required for Lethal Synergism}

These findings revealed that $a g r$ activity was elevated during polymicrobial growth, although it was undetermined whether agr was required for augmented toxin secretion during co-culture with $C$. albicans. Therefore, we assessed toxin secretion of an $\triangle a g r A$ mutant during polymicrobial growth. Using the qualitative blood agar-based and quantitative RBC lysis assays, we confirmed that indeed enhanced toxin expression during co-culture is agrA-dependent, as growth with $C$. albicans was unable to rescue this agr defect (Figure 2-5A, B). Given the strong up-regulation of $\alpha$-toxin observed during in vitro growth with $C$. albicans, we hypothesized that this toxin may drive synergistic lethality during co-infection. Use of an isogenic $\Delta$ hla strain revealed loss of lytic activity against sheep RBCs, indicating that the in vitro lytic phenotype is hladependent (Figure 2-5C). However, complementation of hla on a low copy plasmid $(\Delta h l a-$ phla $)$ restored the lytic phenotype to wild-type levels. Importantly, both the WT and complemented strains exhibited elevated RBC lysis during co-culture with $C$. albicans (Figure 2-5C). An $\alpha$-toxin specific enzyme-linked immunosorbent assay (ELISA) used to assess toxin levels in culture supernatants quantitatively confirmed these results (Figure 2-5D). $\alpha$-toxin was required for early synergistic lethality during IAI as co-infection with the $\Delta h l a$ mutant was avirulent as compared to co-infection with WT and $\Delta h l a$-phla strains (Figure 2-5E). As expected, co-infection with the $\Delta a g r A$ mutant and monomicrobial infections were non-lethal over this same time course (Figure 2-2, Figure 2-5E).

\section{The agr System and $\alpha$-Toxin Production Is Increased During Coinfection}

We next wished to determine whether agr was engaged early during infection prior to presentation of severe symptomatology. Mice were inoculated with either $S$. aureus, $S$. aureus(pDB22), C. albicans and S. aureus, or C. albicans and $S$. aureus(pDB22), sacrificed $8 \mathrm{~h}$ p.i., and underwent peritoneal lavage. Analysis of microbial burden in the lavage fluid revealed that, similar to in vitro culture, staphylococcal burden was similar in both monomicrobial and polymicrobial infections (Figure 2-6A). Microscopic analysis of the lavage fluid revealed the presence of $C$. albicans and $S$. aureus, as well as robust mononuclear infiltrate. The $S$. aureus(pDB22) strain demonstrated agr activity, as evidenced by GFP signal, but only sparingly and mostly associated with resident immune cells (Figure 2-6B). In contrast, $S$. aureus(pDB22) co-inoculated with $C$. albicans demonstrated frequent GFP signal and when juxtaposed near C. albicans (Figure 2-6B). While this result is qualitative, it visually demonstrates that the agr system is indeed engaged in the murine peritoneal cavity. Importantly, at this early time point there was a nearly 3 -fold increase in $\alpha$-toxin (Figure 2-6C) and roughly 4-fold increase in $\mathrm{PGE}_{2}$ (Figure 2-6D) levels observed in 


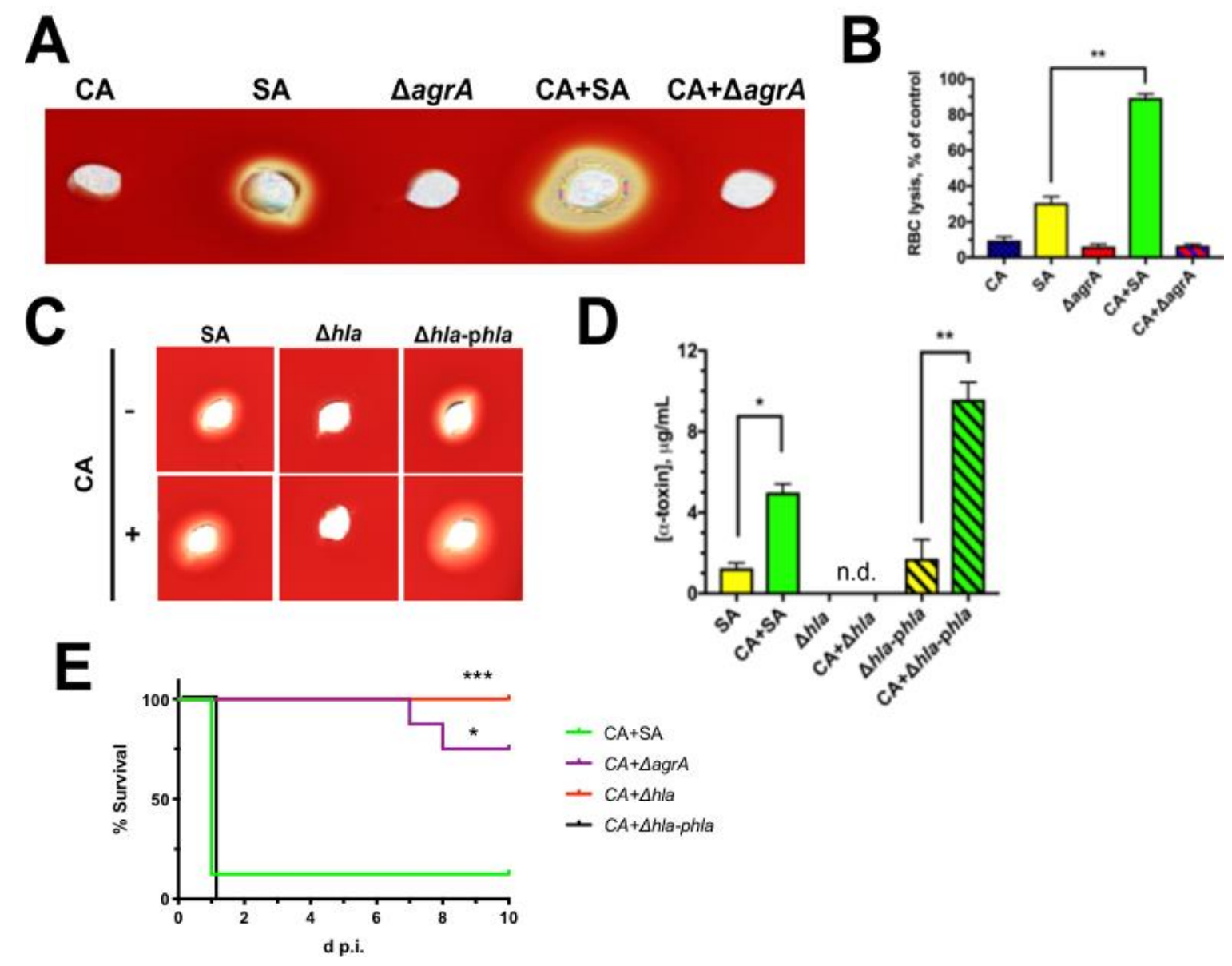

Figure 2-5. Increased $S$. aureus $\alpha$-toxin during growth with $C$. albicans is agrdependent and $\alpha$-toxin is required for lethal infectious synergism during IAI.

(A) Hemolytic activity of monomicrobial and polymicrobial cell-free supernatant $(20 \mu 1$, $16 \mathrm{~h}$ time point) using $S$. aureus (SA) and a $\triangle a g r A$ deletion mutant was assessed using a Sheep's blood agar assay. Representative image of 3 independent repeats. (B) Lytic activity of culture supernatants against rabbit erythrocytes. Data is expressed as the mean (n $=3$ experiments) \pm SEM. *,$P<0.05$. (C) Assay was performed as in panel A using $S$. aureus, $\Delta$ hla deletion mutant, and an hla complemented strain ( $\Delta$ hla-phla).

Representative image of 3 independent repeats. (D) Production of $\alpha$-toxin as measured by ELISA during co-culture of $C$. albicans (green bars) with wild-type $S$. aureus (yellow bar), $\Delta$ hla (red bar, n.d.), or $\Delta$ hla-phla (yellow hashed bar). Data is expressed as the mean \pm SEM. * $P<0.05$; ** $P<0.01$. (E) Mice were co-infected i.p. with $C$. albicans (CA) and wild-type $S$. aureus (green line), $\Delta a g r A$ (purple line), $\Delta$ hla (red line), or $\Delta h l a-$ phla (black line) using established inocula. Mortality was monitored for up to $10 \mathrm{~d}$ p.i. Data is derived from duplicate experiments of $n=4$ mice per group and combined. Significance was assessed using a Wilcoxon rank-sum test. $* * *, P<0.001$. 

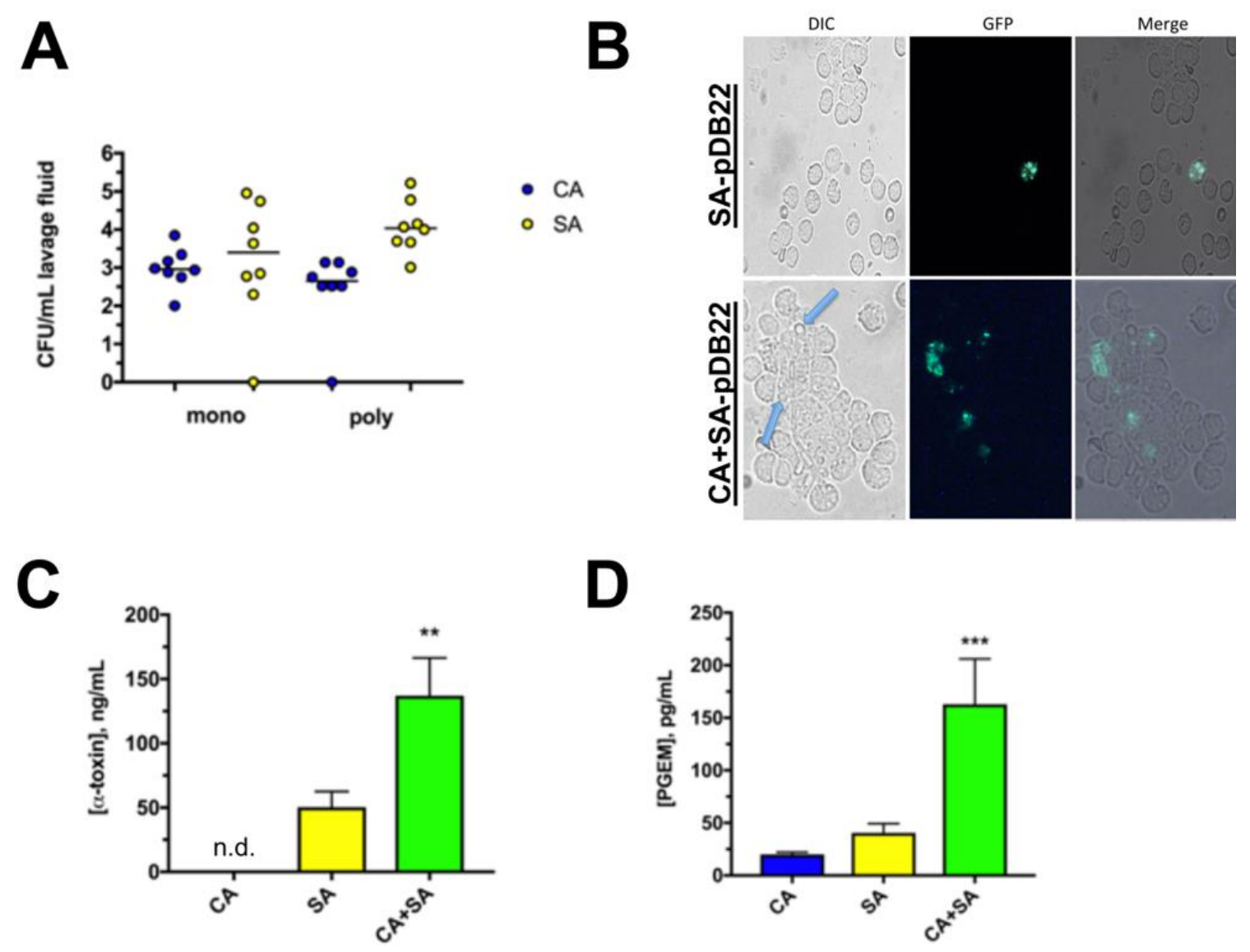

Figure 2-6. The S. aureus agr quorum sensing system is engaged during IAI and polymicrobial infection leads to synergistic increases in $\mathrm{PGE}_{2}$ and $\alpha$-toxin.

(A) Mice ( $\mathrm{n}=4$ per group) were inoculated with S. aureus (SA, yellow) or C. albicans (CA, blue) alone (mono) or with both pathogens simultaneously (poly) using established inocula. Mice were sacrificed $8 \mathrm{~h}$ p.i. and subjected to peritoneal lavage, and lavage fluid was plated on selective media. CFU counts are expressed as the median. (B) Mice were inoculated as described for panel A, except with strain S. aureus(pDB22), containing the GFP-P3 reporter. An aliquot of lavage fluid was assessed $8 \mathrm{~h}$ p.i. by fluorescence microscopy using DIC/GFP filter sets. Blue arrows depict hyphal filaments among peritoneal cells. Images are representative of at least 5 fields of view. (C) Levels of $\alpha$ toxin present in peritoneal lavage fluid $8 \mathrm{~h}$ p.i. during monomicrobial (CA, blue; SA, yellow) and polymicrobial (CA+SA, green) infection as measured by ELISA. Data are cumulative of two independent repeats and expressed as the mean \pm SEM. **, $P<0.01$; n.d., not detected. (D) $\mathrm{PGE}_{2}$, as a measure of PGE metabolites (PGEM), present in the peritoneal lavage fluid $8 \mathrm{~h}$ p.i. as measured by competitive EIA. Data are cumulative of two independent repeats and expressed as the mean \pm SEM. ***, $P<0.001$. Synergistic significance was determined using a Mann-Whitney test (CFU) and Student's $t$ test ( $\alpha$ toxin, PGEM). 
peritoneal lavage fluid of co-infected mice. Mice infected with C. albicans alone demonstrated modest $\mathrm{PGE}_{2}$ and no measurable $\alpha$-toxin in the lavage fluid, demonstrating assay specificity.

\section{Passive Immunization with an Anti- $\alpha$-Toxin Antibody Is Protective Against Synergistic Lethality}

Lastly, we wanted to determine whether blockade of $\alpha$-toxin was sufficient to elicit protection during polymicrobial IAI. Therefore, we passively immunized mice $24 \mathrm{~h}$ prior to co-infection with the anti- $\alpha$-toxin specific monoclonal IgG1 antibody (mAb) MEDI4893* at doses of 15 and $45 \mathrm{mg} / \mathrm{kg}$ or isotype control R347 (82). Mice receiving control antibody succumbed to co-infection within 1 day, similar to that previously observed in untreated mice (Figure 2-7A). Mice receiving MEDI4893* at $15 \mathrm{mg} / \mathrm{kg}$ exhibited a slight delay in mortality ( 2 d p.i.). However, approximately $50 \%$ of these mice succumbed to infection by $3 \mathrm{~d}$ p.i. and this increased to $70 \%$ by $8 \mathrm{~d}$ p.i. Importantly, mice receiving MEDI4893* at a dose of $45 \mathrm{mg} / \mathrm{kg}$ exhibited no mortality until $4 \mathrm{~d}$ p.i. and an overall mortality rate of only $30 \%$ (Figure 2-7A). Thus, passive administration of MEDI4893* improves survival during polymicrobial IAI. We next wanted to determine whether administration of $\alpha$-toxin during infection with $C$. albicans was sufficient to cause synergistic lethality. First, a non-lethal i.p. dose of $\alpha$-toxin $(0.5 \mu \mathrm{g})$ was determined (Figure 2-7B). However, co-inoculation of mice with $C$. albicans and $0.5 \mu \mathrm{g} \alpha$-toxin did not recapitulate the lethal synergy between $C$. albicans and $S$. aureus (Figure 2-7C). Collectively, these results demonstrate that the staphylococcal regulator agr is robustly increased during fungal-bacterial co-infection and that $\alpha$-toxin is necessary but not sufficient in mediating early lethal synergism with $C$. albicans.

\section{Conclusions}

In nature, microorganisms rarely grow as single species but instead most exist as polymicrobial communities characterized by mutualistic, parasitic, commensalistic, or antagonistic interactions. With the advent of high throughput sequencing technologies, we have now begun to realize the tremendous microbial diversity that exists, not only in the environment, but also within the human biome. Although we are rapidly amassing incredible amounts of data regarding microbiome composition, functional and mechanistic relationships between microbial consortia and human host are only now being explored in depth. Furthermore, the boundaries of Koch's postulates are being challenged, as some disease phenotypes are now realized to result from a complex set of microbial virulence mechanisms, partially governed by microbe-microbe interactions. One extreme example is "lethal synergism", in which co-infection mediates mortality of otherwise survivable infections with each microbe independently. This phenomenon has been previously reported in a variety of biological systems but is perhaps best characterized by fungal-bacterial IAI with $C$. albicans and $S$. aureus. 


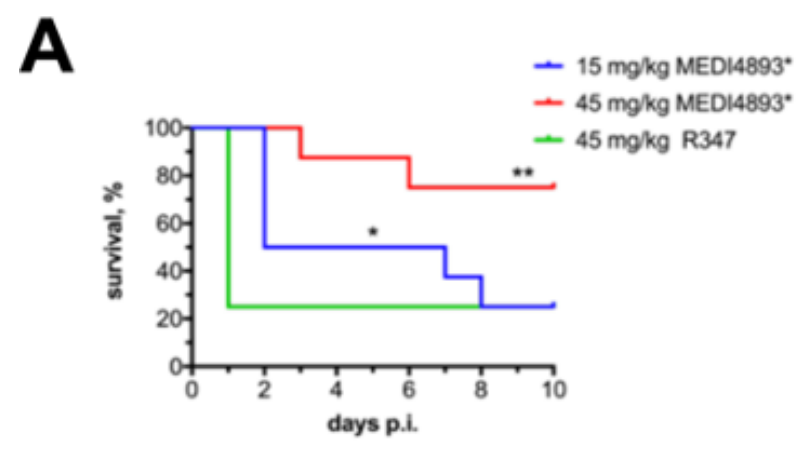

B

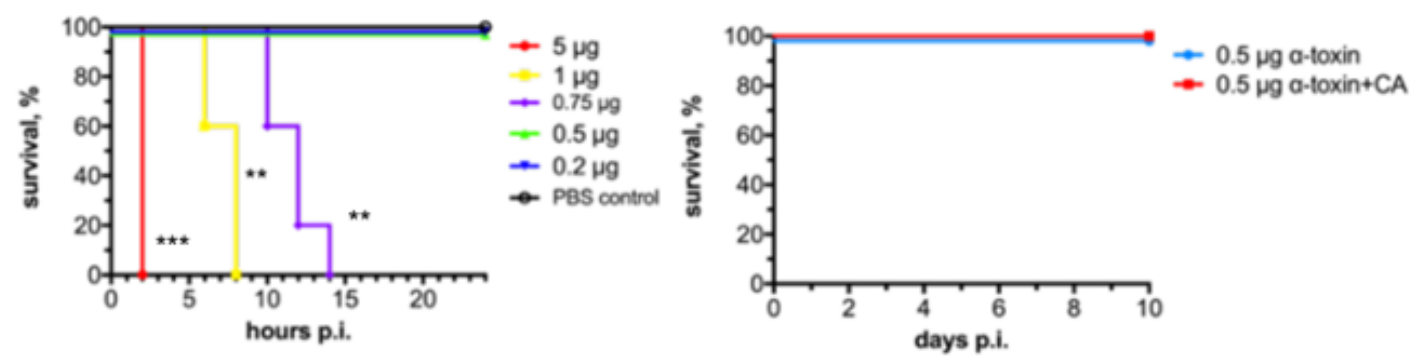

Figure 2-7. $\alpha$-toxin is necessary but not sufficient to drive infectious synergism during IAI.

(A) Mice ( $\mathrm{n}=4$ per group) were inoculated i.p. with $45 \mathrm{mg} / \mathrm{kg}$ human IgG1 isotype control antibody R347 (green) or an IgG1 antibody specific for $\alpha$-toxin (MEDI4893*) at doses of $15 \mathrm{mg} / \mathrm{kg}$ (blue) or $45 \mathrm{mg} / \mathrm{kg}$ (red) $24 \mathrm{~h}$ prior to i.p. coinfection with C. albicans and S. aureus. Survival was monitored for up to $10 \mathrm{~d}$ p.i. Data are from duplicate experiments of 4 mice per group and were combined. Significance was assessed using a Wilcoxon log rank test. (B) A range of $\alpha$-toxin doses were injected i.p. into groups of mice $(n=4)$ to determine lethality. (C) Swiss-Webster mice were inoculated i.p. with 0.5 $\mu \mathrm{g}$ of $\alpha$-toxin, immediately followed by i.p. injection of either sham PBS (blue) or $C$. albicans (red). Data are representative of duplicate experiments with 4 mice per group, and data were combined. *, $P<0.05$; ***, $P<0.01$; ***, $P<0.001$. 
Our lab has explored the interaction between $C$. albicans and $S$. aureus extensively. Previous studies revealed that indeed, $C$. albicans influenced global protein expression during growth with $S$. aureus, including modulation of several putative virulence factors (47). It was also determined that $S$. aureus preferentially binds the invasive hyphal form of $C$. albicans via the candidal adhesin Als3p and this interaction allows for enhanced mortality and systemic dissemination from the oral cavity during oropharyngeal candidiasis. Furthermore, during biofilm growth, $C$. albicans confers highlevel vancomycin resistance to $S$. aureus by forming a drug permeability barrier via elaboration of carbohydrate dense extracellular matrix $(46,48)$. These studies highlight the highly dynamic relationship between these microbes.

Data in this manuscript build off of recent efforts by our lab seeking to define the mechanism by which $C$. albicans augments $S$. aureus infection during polymicrobial IAI, as observed by Carlson et al. many years prior. Initial studies determined that host inflammation, including the eicosanoid $\mathrm{PGE}_{2}$ and cytokine IL-6, was synergistically exacerbated in mice challenged with both microbes simultaneously as compared to monomicrobial infection and correlated with lethal outcome (67). Furthermore, protection was mediated by anti-inflammatory administration and subsequent $\mathrm{PGE}_{2}$ blockade $(62,87)$. Using various transcriptional regulator mutants, lethal infection was surprisingly independent of $C$. albicans morphological transition, as other experimental C. albicans disease models are almost universally dependent on morphogenetic regulation (66). This observation was further confirmed by the fact that various other Candida species mediate synergistic lethality independent of capacity to form true hyphae during IAI (67). Importantly, all of these studies were conducted using S. aureus strain NRS383 (USA200), which is grouped into the CC30 clonal complex (50). While synergistic lethality was achieved during co-infection with this strain, kinetics $(\sim 3 \mathrm{~d}$ p.i.) were modest compared to infection with JE2 ( $<1 \mathrm{~d}$ p.i.) in this study. Interestingly, CC30 strains are documented to have very low to no production of $\alpha$-toxin under in vitro conditions, in some cases due to a premature stop codon within hla (103). NRS383 exhibits a weak zone of hemolysis on blood agar, further emphasizing the importance of staphylococcal $\alpha$-toxin in driving lethal synergism with $C$. albicans.

The major staphylococcal regulator of $\alpha$-toxin production is indeed governed by agr-dependent signaling. However, several reports suggest contribution of agrindependent mechanisms regulated via the sarA and saeRS networks (104). In support of this, clinical isolates that are agr negative have been observed to activate hla transcription in vivo. Similarly, deletion of sae causes loss of hla transcription, despite an intact agr regulon (105). Results from our previous study of polymicrobial IAI demonstrate that $S$. aureus disseminates to distant tissues (including the brain) quickly and, at later time points reaches greater numbers during polymicrobial as compared to monomicrobial infection (66). Thus, it is possible that delayed activation of these alternative $\alpha$-toxin regulators at sites of dissemination in vivo may explain lethality with strains exhibiting low hemolytic activity in vitro. Given that a relatively large fraction (15-60\%) of HAMRSA strains are $a g r$-defective, it will be interesting to delineate the role of these alternative $\alpha$-toxin regulators in driving synergistic lethality and their potential activation during growth with $C$. albicans (106). 
Experiments utilizing the isogenic $\Delta h l a$ strain during co-infection demonstrated a clear requirement for $\alpha$-toxin to mediate infectious synergism. These findings were corroborated by Rauch et al, in which loss of hla during high-dose monomicrobial peritoneal challenge with $S$. aureus strain Newman dramatically improved survival rates as compared to wild-type infection (97). Moreover, blockade of $\alpha$-toxin using the investigational neutralizing antibody MEDI4893* further confirmed the importance of this virulence determinant in driving mortality during IAI. MEDI4893* exerts its effects by not only inhibiting engagement of $\alpha$-toxin to its host receptor ADAM10, but also by preventing oligomerization of the mature heptamer required for membrane pore formation and lytic activity (107). Aside from the clear protective effects demonstrated against polymicrobial IAI in this study, MEDI4893* has also demonstrated efficacy in both immunocompetent and immunocompromised animal models, including necrotizing pneumonia, dermonecrosis, and sepsis $(82,108-110)$. Although prophylactic treatment with MEDI4893* did not confer $100 \%$ survival in this study, it did significantly delay mortality to $2(15 \mathrm{mg} / \mathrm{kg}$ ) and 4 d p.i. (45 mg/kg), respectively, which could allow for an extended therapeutic window for antimicrobial administration. Although beyond the scope of this work, future studies to determine whether MEDI4893* can be given therapeutically to confer protection post-infection are warranted. In any case, passive immunization against $\alpha$-toxin is an intriguing possibility for those at elevated risk of IAI, including abdominal surgery, peritoneal dialysis, or tertiary peritonitis patients.

Cohen et al. recently demonstrated that $\alpha$-toxin is necessary and sufficient for $S$. aureus lethal synergy during lung co-infection with Gram-negative opportunists (111). However, it is still unclear whether $\alpha$-toxin is sufficient to mediate lethal synergism during $C$. albicans infection or if other components of $S$. aureus are also required. Carlson had previously observed that inoculation of $C$. albicans with cell-free spent culture supernatants of $S$. aureus could induce synergistic lethality, suggesting that staphylococcal secreted products (e.g. toxins) were perhaps implicated (72). The potency of $\alpha$-toxin is impressive, with the $\mathrm{LD}_{50}$ being reported as $\sim 1 \mu \mathrm{g}$ via systemic injection or intranasal challenge; an intradermal dose of $10 \mu \mathrm{g}$ is sufficient to induce large skin lesions $(82,84,112)$. Despite initial signs of morbidity, inoculation of mice with $C$. albicans followed by a sub-lethal dose of purified $\alpha$-toxin was unable to recapitulate synergistic mortality (Figure 2-7). There are several reasons why this may be the case. It is possible that a sub-lethal bolus dose of toxin is not sufficient to potentiate effects with C. albicans. It is also equally likely that sustained expression of $S$. aureus toxin during infection is required to augment co-infection. Alternatively, $\alpha$-toxin may only potentiate host- or microbe-derived signals present during infection with intact fungal or bacterial pathogens or may be required to be present with $C$. albicans at the immunological synapse. Additionally, Carlson's observations may be explained by the presence of multiple toxins or shed cell wall material present in the culture supernatant that were not completely inactivated by heating. These soluble factors may maintain pro-inflammatory activity to elicit robust $\mathrm{PGE}_{2}$ and cytokine production during co-infection by stimulating pattern recognition receptors (PRRs) and serving as danger-associated molecular patterns (DAMPs). 
This study describes the novel finding of a human pathogenic fungus engaging virulence of a bacterial pathogen with dire consequences for the host. That said, crossspecies and cross-domain quorum sensing communication has been observed in several other reports. For example, Bamford, et al. demonstrated that the diffusible chemical signal AI-2, derived from the luxS gene of the ubiquitous oral commensal Streptococcus gordonii, enhances $C$. albicans hypha formation during polymicrobial biofilm growth (113). Perhaps one of the best well-studied interactions occurs between $C$. albicans and Pseudomonas aeruginosa, whereby the bacterial quorum sensing molecule 3-oxo-C12 inhibits the fungal yeast-to-hypha transition (114). P. aeruginosa also attaches to the fungal surface, secreting a variety of fungicidal substances (phenazine, pyocyanin, etc.) (15). In response, $C$. albicans secretes its own quorum sensing compound farnesol that has antibacterial activity (25). Therefore, it is not unreasonable to speculate that secreted fungal quorum sensing molecules (or otherwise) may elicit toxigenic responses by $S$. aureus via quorum sensing induction. Indeed, at supra-physiologic concentrations, farnesol does exhibit anti-staphylococcal effects (27). Although apparent lack of an antagonistic relationship between these microbes during co-culture, as evidenced by previous imaging studies, further clouds this hypothesis (47).

It is possible that cell-cell contact, and not necessarily a secreted factor, mediates the toxigenic response in $S$. aureus. Previous studies from our lab have demonstrated that the major adhesin responsible for $C$. albicans-S. aureus interactions is the candidal protein Als3p (50). This fact, coupled with the ability of several transcriptional regulator mutants of $C$. albicans incapable of hypha formation to still promote lethal synergism during IAI, suggests an alternative mechanism as these strains would also be predicted to not express hypha-associated Als3p $(66,67)$. Furthermore, co-infection with a $\Delta / \Delta$ als 3 mutant of $C$. albicans still exhibited synergistic lethality with $S$. aureus (JE2) during experimental IAI (unpublished studies). That said, maximal engagement of a soluble factor to its potential target in vivo may occur if both microbes maintain intimate contact. We also cannot rule out the possibility that an environmental factor or host component mediates enhanced toxin secretion in vivo. It is well known that the $a g r$ system is sensitive to several environmental stimuli, including $\mathrm{pH}$; the $\mathrm{pH}$ of the abdominal cavity during $C$. albicans peritonitis is slightly alkaline $(68,115)$. Thus, it is possible that anatomical niches within the abdomen buffer the acidic end products of staphylococcal metabolism, thereby maintaining neutral $\mathrm{pH}$ levels optimal for sustained agr activation. $C$. albicans infection may also generate host or fungal byproducts that $S$. aureus can use as metabolic substrates or signaling molecules to up-regulate toxin expression.

Collectively, these results demonstrate that $C$. albicans augments $S$. aureus virulence via an $a g r$ - and $\alpha$-toxin-dependent mechanism during polymicrobial IAI, resulting in devastating consequences for the host. These findings are important given the frequency with which these microbes are co-isolated from biologic (e.g. vagina, gastrointestinal tract, skin, oropharynx) and artificial (e.g. indwelling catheters) sites during colonization or infection. Furthermore, systemic disease caused by Candida species is associated with mortality rates exceeding $40 \%$ even with appropriate therapy (116). Given our observations, it is possible that these unacceptably high mortality rates may not only result from fungal infection alone, but may be engaging virulence 
mechanisms in otherwise commensal microbes or co-infecting pathogens $(60,117,118)$. Novel strategies to inhibit fungal-bacterial quorum sensing cross-talk or its downstream effectors may be warranted to better manage IAI, including use of an antibody-mediated vaccine against staphylococcal $\alpha$-toxin in at risk patient populations to limit incidence of severe sepsis. 


\section{CHAPTER 3. CANDIDA ALBICANS IMPACTS STAPHYLOCOCCUS AUREUS $\alpha$-TOXIN PRODUCTION VIA EXTRACELLULAR ALKALINIZATION*}

\section{Introduction}

Candida albicans, an opportunistic polymorphic fungus, and Staphylococcus aureus, a ubiquitous bacterial pathogen, rank among the top organisms responsible for life-threatening invasive disease. Not only do these pathogens cause significant morbidity and mortality on their own, evidence for their existence as part of polymicrobial consortia has surfaced. For instance, $C$. albicans and $S$. aureus have been co-isolated from a variety of biotic and abiotic surfaces, including central venous catheters, prosthetic implants, the skin, and mucosal layers $(75,119)$. Moreover, they have been co-associated with several polymicrobial diseases, including burn wound superinfection, ventilator-associated pneumonia, urinary tract infection, cystic fibrosis, and bloodstream infection (42-44, 120). However, perhaps the most comprehensive line of investigation of this particular co-infection has focused on their role during intra-abdominal infection (IAI).

IAI is a spectrum of diseases characterized by microbial invasion and subsequent inflammation of the abdominal cavity (55). Mortality rates for such infections typically range between 10-30\%; however, mortality rates involving a fungal pathogen (e.g. $C$. albicans) can approach $80 \%$, even with appropriate treatment $(56,57)$. Using a murine model of IAI, a series of studies by Carlson, et al. demonstrated that $C$. albicans enhanced the virulence of $S$. aureus, as co-infection reached $100 \%$ mortality within days p.i. while monomicrobial infection with either pathogen was non-lethal (59). Studies designed to titrate various inoculating doses of $C$. albicans and $S$. aureus during coinfection revealed that this apparent synergism was not mutual, as $C$. albicans virulence was not augmented by low doses of $S$. aureus (60). Synergistic mortality rates were found to be dependent on various toxins produced by $S$. aureus (71). Although these studies failed to delineate which specific $S$. aureus toxin was responsible, they established their important role in driving pathogenicity in the context of polymicrobial IAI.

Toxin expression in $S$. aureus is governed by a complex set of transcriptional regulators that respond to endogenous and environmental stimuli. Perhaps the best wellcharacterized mechanism is driven by the accessory gene regulator ( $a g r$ ) quorum sensing system that is activated in a cell density-dependent manner. The agr operon is composed of four genes encoding for AgrA, AgrB, AgrC, and AgrD proteins (121). AgrD serves as the immature signal peptide that is proteolytically processed and secreted by membrane bound AgrB, releasing the mature signal molecule auto-inducing peptide 2 (AIP-2). AIP2 can be sensed by the surface bound receptor and histidine kinase AgrC that phosphorylates and activates the transcription factor AgrA. Activation of AgrA

*Reprinted from final submission with open access permission. Todd OA, Noverr MC, Peters BM. 2019. Candida albicans Impacts Staphylococcus aureus Alpha-Toxin Production via Extracellular Alkalinization. mSphere 4.(122). 
simultaneously up-regulates expression of the agr operon completing a positive feedback loop, while also ultimately down-regulating colonization factors (e.g. adhesins) and upregulating virulence factors including toxins $(80,121)$. While agr is undoubtedly induced as a consequence of quorum development, it is also highly susceptible to environmental factors including high salt, glucose, subinhibitory antibiotic concentrations, and $\mathrm{pH}$ (115, $123)$.

Our laboratory has recently demonstrated that during in vitro growth, C. albicans has the capacity to activate the agr regulon, leading to exacerbated production of $\alpha$-toxin, a potent staphylococcal virulence determinant capable of lysing a variety of host cells, causing tight-junction loss, and activating numerous innate pro-inflammatory pathways (65). Moreover, this virulence factor was crucial for driving lethal synergism during polymicrobial IAI. By using a combination of genetic and functional assays, the objective of this study was to attempt to elucidate the mechanism by which $C$. albicans activates agr signaling and $\alpha$-toxin production by $S$. aureus and to determine the extent that other non-albicans Candida (NAC) species can augment $\alpha$-toxin release (65). In the course of conducting these studies, we discovered that modulation of the extracellular $\mathrm{pH}$ by $C$. albicans creates an optimal environment for robust induction of the staphylococcal agr system further highlighting how complex ecological signals may intersect with virulence during this prevalent fungal-bacterial interaction.

\section{Materials and Methods}

\section{Strains and Growth Conditions}

Candida albicans SC5314 (referred to as CA) was used as the wild-type isolate throughout this work. The alkalinization-deficient mutant $s t p 2 \Delta / \Delta$ (SVC17) and its isogenic revertant strain STP2-REV (SVC19) were kind gifts from Dr. Michael Lorenz (University of Texas Health Science Center) (124). The non-albicans Candida (NAC) species C. glabrata CBS138 (CG), C. dubliniensis CD36 (CD), C. parapsilosis CDC317 (CP), C. tropicalis MYA3404 (CT), and C. krusei 81-B-5 (CK) were used as speciesrepresentative strains (67). JE2, a USA300 isolate, was obtained from the Biodefense and Emerging Infectious (BEI) Research Resources repository and used as the wild-type $S$. aureus strain in this work and is referred to as SA. A $S$. aureus reporter strain $[S$. aureus(pDB22)] containing plasmid pDB22 (containing the $\mathrm{P} 3$ promoter fused to GFPmut2 and an erythromycin resistance cassette) was also used in this work (125). All strains were maintained as $20 \%$ glycerol stocks at $-80^{\circ} \mathrm{C}$.

Candida strains were streaked onto yeast peptone dextrose (YPD) agar and grown at $30^{\circ} \mathrm{C}$. A single colony was inoculated in YPD broth and incubated overnight at $30^{\circ} \mathrm{C}$ with shaking at $200 \mathrm{rpm}$. S. aureus strains were streaked on trypticase soy agar (TSA) (with $10 \mu \mathrm{g} / \mathrm{mL}$ erythromycin added as needed). Single colonies were inoculated in TSB and grown overnight at $37^{\circ} \mathrm{C}$ with shaking at $200 \mathrm{rpm}$. Aliquots $(500 \mu \mathrm{L})$ were washed

$3 \mathrm{X}$ with PBS and cell concentrations adjusted to $1 \times 10^{7} \mathrm{CFU} / \mathrm{mL}$. A 1:100 dilution was 
made into $5 \mathrm{~mL} 0.6 \mathrm{X} \mathrm{TSB}+0.2 \%$ glucose (TSB-g) with the following groups: CA or SA (monomicrobial) and CA+SA (polymicrobial). PBS $(50 \mu \mathrm{L})$ was added to monomicrobial cultures. Cultures were incubated at $37^{\circ} \mathrm{C}$ with shaking at $200 \mathrm{rpm}$ and aliquots removed at $16 \mathrm{~h}$ post-inoculation (p.i.). Mono- and polymicrobial cultures using the alkalinizationdeficient mutant and revertant strains were prepared as above, except TSB-g was adjusted to $\mathrm{pH} 6$ prior to inoculation and cultures were incubated for $24 \mathrm{~h}$.

\section{agr Reporter Assay}

Mono- and polymicrobial cultures were prepared as described above using CA and/or $S$. aureus(pDB22) (10 $\mu \mathrm{g} / \mathrm{mL}$ erythromycin added for plasmid maintenance). At $16 \mathrm{~h}$ p.i., $100 \mu \mathrm{L}$ aliquots were removed in triplicate from cultures and added to wells of 96-well black microtiter plate. Fluorescence (488 nm excitation, $525 \mathrm{~nm}$ emission) was measured using a plate reader (Synergy, Biotek). Experiments were repeated in triplicate and results expressed as the mean arbitrary fluorescence units (AFU) \pm SEM.

\section{Blood Agar Lysis Assay}

Cultures were prepared as described above and at $16 \mathrm{~h}$ p.i., $5 \mathrm{~mL}$ aliquots were centrifuged at $5000 \mathrm{rpm}$ to pellet cells, and supernatants sterilized using $0.2 \mu \mathrm{m}$ syringe filters. Sterile supernatants were concentrated 20x by ethanol precipitation. Holes were punched in blood agar plates (TSA with 5\% sheep's blood) using a sterile pipette tip. Concentrated supernatant $(20 \mu \mathrm{L})$ was added to wells and plates were incubated at $37^{\circ} \mathrm{C}$ for $24 \mathrm{~h}$. Plates were photographed using a digital scanner (EPSON Perfection V700 Photo).

\section{pH Buffering}

$\mathrm{pH}$ buffering experiments were done by the same culture set-up described above, with the following changes: $100 \mathrm{mM}$ MOPS was added to $0.6 \mathrm{X}$ TSB $+0.2 \%$ glucose and the media was adjusted to varying $\mathrm{pH}(5.5,6,6.5,7,7.5$, and 8) using $5 \mathrm{~N} \mathrm{HCl}$ or $\mathrm{NaOH}$ as required. Aliquots $(500 \mu \mathrm{L})$ of overnight cultures of CA and $S$. aureus $(\mathrm{pDB} 22)$ were washed with PBS and adjusted to $1 \times 10^{7} \mathrm{CFU} / \mathrm{mL}$. A 1:100 dilution was made into 5 $\mathrm{mL}$ MOPS-buffered TSBg at each $\mathrm{pH}$ tested. Mono- and polymicrobial cultures were incubated for $16 \mathrm{~h}$ at $37^{\circ} \mathrm{C}$ with shaking at $200 \mathrm{rpm}$.

\section{Kinetic agr Activation Assay}

S. aureus(pDB22) was grown overnight in MOPS-buffered 0.6X TSB-g, pH 5.5 to inactivate agr. Additionally, S. aureus (JE2) and C. albicans were grown overnight in MOPS-buffered 0.6X TSB-g, pH 7. Cultures were centrifuged at $4000 \mathrm{rpm}$ for $3 \mathrm{~min}$. Supernatant from the JE2 and C. albicans cultures were collected and filter sterilized. 
Cells from $S$. aureus(pDB22) grown at $\mathrm{pH} 5.5$ were washed with $\mathrm{PBS}$ and adjusted to $1 \mathrm{x}$ $10^{10} \mathrm{CFU} / \mathrm{mL}$. Cells were added to $1 \mathrm{~mL}$ 5X MOPS-buffered TSB-g, $\mathrm{pH} 7$ to a final concentration of $2 \times 10^{8} \mathrm{CFU} / \mathrm{mL} .4 \mathrm{~mL}$ of either $S$. aureus spent, $C$. albicans spent, or fresh media was added to the cells along with $10 \mu \mathrm{g} / \mathrm{mL}$ erythromycin (for plasmid maintenance). Cultures were incubated at $37^{\circ} \mathrm{C}$ with shaking at $200 \mathrm{rpm}$. At $20 \mathrm{~min}$ intervals, $100 \mu \mathrm{L}$ aliquots were removed and fluorescence was measured as above. Experiments were repeated in triplicate and results expressed as the mean AFU \pm SEM.

\section{$\alpha$-Toxin ELISA}

S. aureus $\alpha$-toxin was quantified by ELISA, as described previously (65). Briefly, 96-well plates were coated with $0.1 \mu \mathrm{g} / \mathrm{mL}$ MEDI4893* diluted in coating buffer and incubated overnight at $4^{\circ} \mathrm{C}$. All wash steps were carried out with PBS- $0.05 \%$ Tween 20 (PBS-T). Plates were washed and blocked with SuperBlock (Pierce) for $1 \mathrm{~h}$ at room temperature. $50 \mu \mathrm{L}$ of diluted filter-sterilized supernatant (taken at $16 \mathrm{~h}$ p.i.) was added to wells, with serial dilutions of native alpha-toxin included as the standard curve. The plates were incubated for $1 \mathrm{~h}$ at room temperature and then washed. $2 \mu \mathrm{g} / \mathrm{mL}$ affinitypurified rabbit polyclonal anti- $\alpha$-toxin antibody was added to wells and the plates were incubated for $1 \mathrm{~h}$ at room temperature. Plates were washed, followed by addition of a 1:10,000 dilution of Affinipure HRP-coupled goat anti-rabbit IgG detection antibody (Jackson Immuno Research). Plates were incubated for $1 \mathrm{~h}$ at room temperature and washed. TMB substrate was added to wells and color was allowed to develop in the dark for $10 \mathrm{~min}$. $100 \mu \mathrm{L}$ ELISA stop solution $\left(0.2 \mathrm{M} \mathrm{H}_{2} \mathrm{SO}_{4}\right)$ was added and wells were read at $450 \mathrm{~nm}$ using a spectrophotometer (Synergy, Biotek). Culture supernatants from $S$. aureus NE1354 $\Delta$ hla were used as background controls and were subtracted from sample wells to exclude any non-specific binding of antibody by protein A. The experimental values were extrapolated to the standard curve. Experiments were completed in triplicate and shown as mean concentration \pm SEM.

\section{CFU Analysis}

CFU enumeration was done by serial plating of culture media onto YPD containing $20 \mu \mathrm{g} / \mathrm{mL}$ nafcillin (for Candida enumeration) and TSA containing $2.5 \mu \mathrm{g} / \mathrm{mL}$ amphotericin B (for $S$. aureus enumeration) via the drop-plate method (48). Plates were incubated overnight at $37^{\circ} \mathrm{C}$, enumerated, and expressed as $\mathrm{CFU} / \mathrm{mL}$. CFU values are representative of 3 independent repeats and represented as median $\pm \mathrm{SEM}$. 


\section{Results}

\section{C. albicans Augments Staphylococcal $\alpha$-Toxin and Does Not Complement agr Signaling in Trans}

Similar to previously reported findings (65), co-culture of $C$. albicans and $S$. aureus led to elevated hemolytic toxin production when compared to mono culture, as assessed by a functional hemolytic assay on Sheep's blood agar (Figure 3-1A). Unsurprisingly, monoculture of C. albicans did not demonstrate lysis as this fungus is not commonly reported to lyse red blood cells on microbiological agar (Figure 3-1A). While the regulation of virulence factor production in S. aureus is multi-factorial, the agr quorum sensing system plays a major role in governing increased toxin expression. In order to confirm that the agr system was more robustly activated during co-culture, a P3GFP reporter system (the $\mathrm{P} 3$ promoter is a target of phosphorylated and activated AgrA and ultimate driver of toxin expression) was employed. Indeed, reporter analysis indicated approximately a 2.5 -fold induction of $\operatorname{agr}$ (Figure 3-1B), which correlated with $\sim 4$-fold production of $\alpha$-toxin as measured by specific ELISA (Figure 3-1C). These results were consistent with those in Figure 3-1A given that the hemolytic phenotype observed on Sheep's blood agar is dependent on $\alpha$-toxin activity.

The next logical line of investigation was to determine whether $C$. albicans may be producing a protein or other small molecule that could be activating the $a g r$ system in S. aureus leading to up-regulation of toxin. Loss of agrA is predicted to largely ablate quorum sensing and elevated toxin production as its activated form binds to both P2 and P3 promoters to drive the agr regulon and decrease repressor of toxin (rot), respectively. However, deletion of $\operatorname{agr} B$ would theoretically only attenuate secretion of the quorum signal peptide auto-inducing peptide 2 (AIP-2) (30-32). During monoculture, this would disrupt sensing of the quorum signal via AgrC and negatively impact toxin production. However, if $C$. albicans produced a molecule(s) that could be sensed by AgrC then the native regulatory circuit could be bypassed during co-culture, resulting in elevated toxin expression. Therefore, similar co-culture assays were conducted as in Figure 3-1, this time including isogenic $\triangle a g r A$ and $\triangle a g r B$ mutants. As predicted, disruption of agrA led to nearly a complete loss of hemolysis on Sheep's blood (Figure 3-2A) and significantly reduced levels of $\alpha$-toxin (Figure 3-2B). Moreover, deletion of agrB demonstrated similar toxin phenotypes as compared to the $\triangle a g r A$ mutant (Figure 3-2A, B). These results suggested that an intact $a g r$ regulon is required for elevated toxin expression. However, the augmented toxin phenotype was unlikely mediated by a fungal ligandbacterial receptor interaction governed through AgrC sensing.

\section{A Role for Extracellular pH in Modulating agr Signaling During Coculture}

Previous reports have determined that regulation of agr is influenced by a number of physiologic factors, including low extracellular $\mathrm{pH}(115,123)$. Therefore, we assessed the $\mathrm{pH}$ of mono- and co-cultures following standard growth conditions. The $\mathrm{pH}$ of 
A

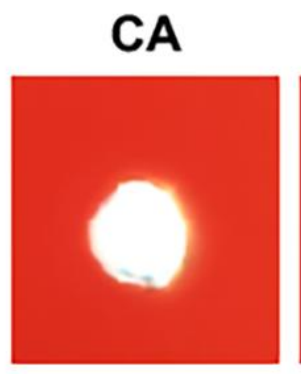

SA
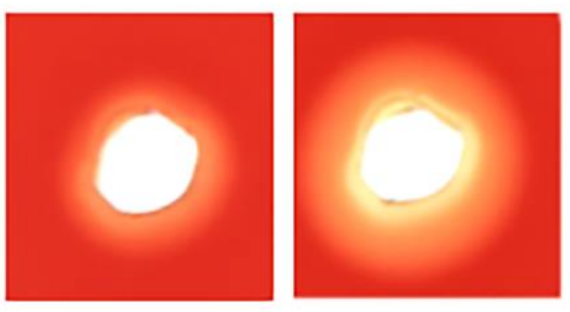

B
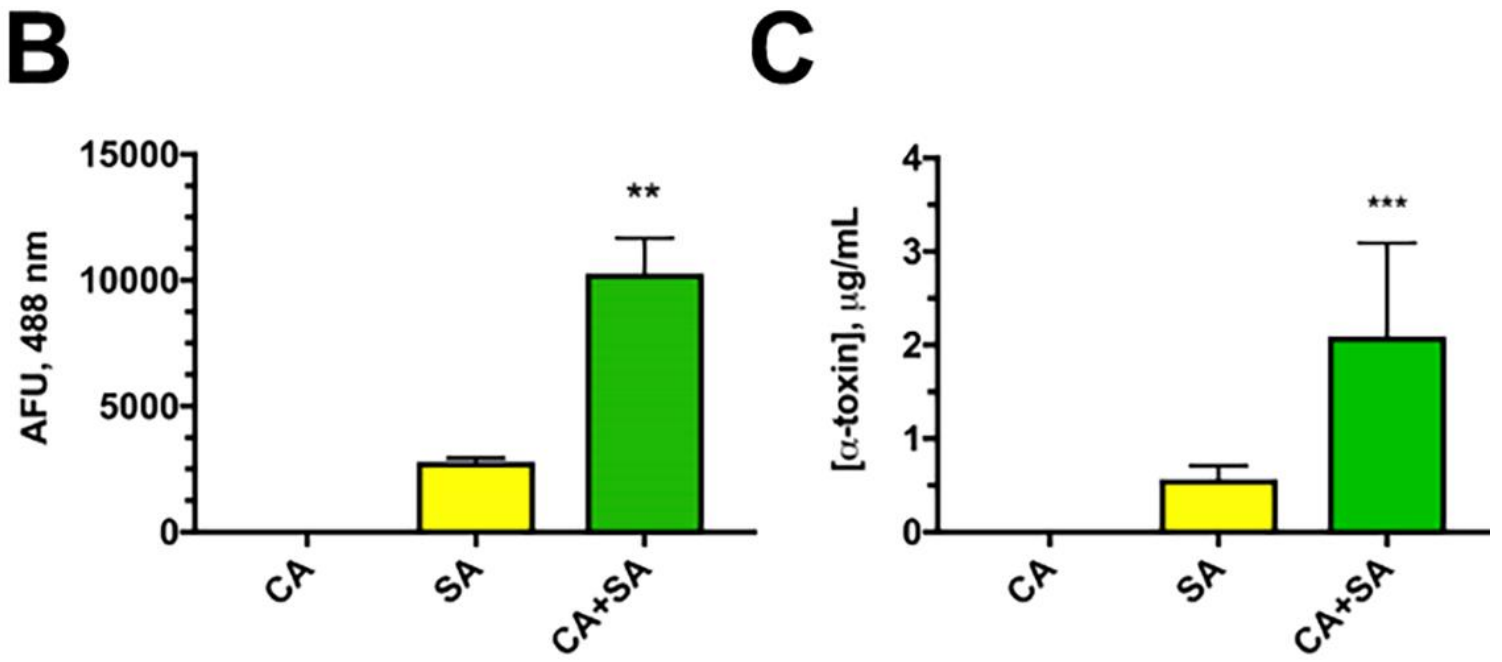

Figure 3-1. C. albicans enhances $S$. aureus $\alpha$-toxin production.

(A) Hemolytic activity of monomicrobial C. albicans (CA, blue), S. aureus (SA, yellow) and polymicrobial cultures (CA+SA, green) was functionally assessed by depositing 20 $\mu \mathrm{L}$ of cell-free culture supernatants into wells on a Sheep's blood agar plate. Images were taken after incubation at $37^{\circ} \mathrm{C}$ for $24 \mathrm{~h}$ using a digital scanner. (B) A P3-GFP reporter strain of $S$. aureus was incubated alone or with C. albicans. After 16 h of growth, $100 \mu \mathrm{L}$ culture was removed in triplicate, added to a 96-well plate, and the fluorescence measured at 488/515 $\mathrm{nm}$ on a fluorimeter. (C) Concentration of $\alpha$-toxin in supernatants from monomicrobial and polymicrobial cultures was measured by ELISA. Data is representative of 3 independent repeats and expressed as the mean \pm SEM. Data was assessed for significance using one-way ANOVA and Dunnett's posttest. **, $P<0.01$; $* * *, P<0.001$. 

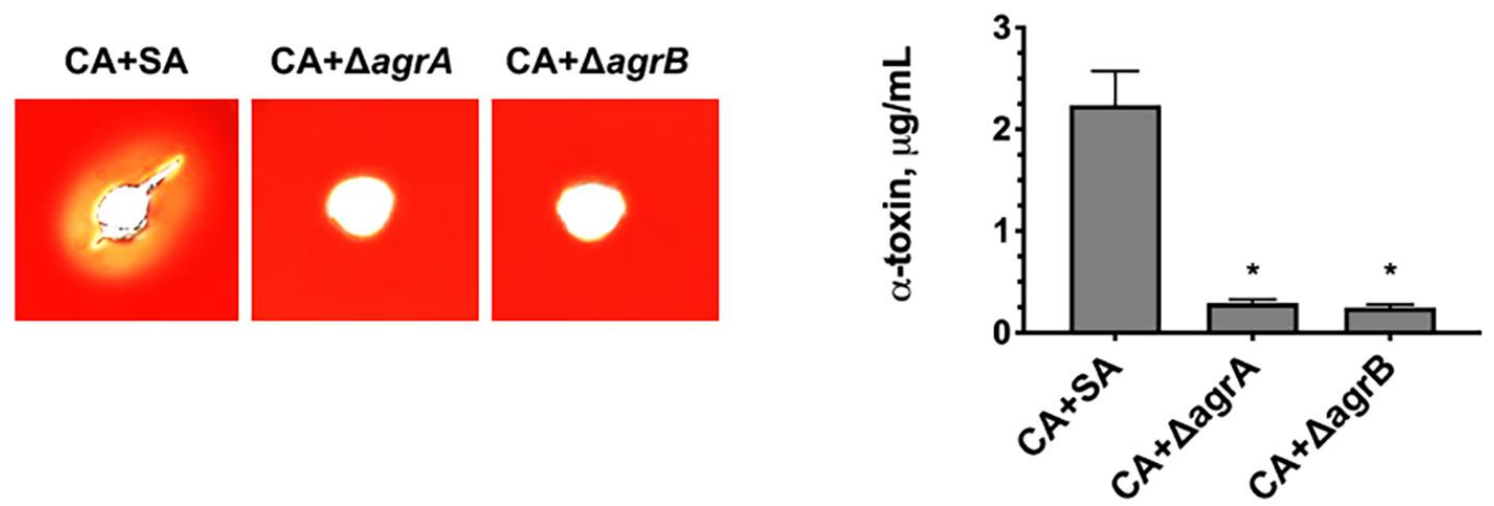

Figure 3-2. Candida-enhanced toxin production is dependent on intact staphylococcal agrA and $\operatorname{agrB}$ signaling.

(A) Hemolytic activity of polymicrobial cultures of $C$. albicans (CA) with wild-type $S$. aureus (SA) or isogenic strains lacking agr genes ( $\triangle a g r A$ or $\triangle a g r B)$ was functionally assessed by depositing $20 \mu \mathrm{L}$ of cell-free culture supernatants into wells on a Sheep's blood agar plate. Images were taken after incubation at $37^{\circ} \mathrm{C}$ for $24 \mathrm{~h}$ using a digital scanner. (B) Levels of $\alpha$-toxin were measured in polymicrobial culture supernatants by ELISA. Data are representative of 3 independent repeats and expressed as the mean \pm SEM. Data was assessed for significance using one-way ANOVA and Dunnett's posttest. $*, P<0.05$. 
C. albicans monoculture was estimated to be $\sim 7.5 \pm 0.1$, while that of $S$. aureus monoculture was $\sim 5.2 \pm 0.2$ (Figure 3-3A). Interestingly, the $\mathrm{pH}$ of the co-culture was $\sim 6.7 \pm 0.2$ (Figure 3-3A). The $\mathrm{pH}$ of fresh TSB-g was determined to be $7.2 \pm 0.2$. Therefore, it appeared as though $C$. albicans was elevating or maintaining the $\mathrm{pH}$ in a range which is optimal for $a g r$ activation. We next determined whether enhanced $a g r$ activity during co-culture could be overridden by experimental modulation of the $\mathrm{pH}$ via buffering TSB-g with $100 \mathrm{mM}$ MOPS. We specifically chose to assess $\mathrm{pH}$ points that closely matched to those representing $C$. albicans mono, $S$. aureus mono, and co-culture values. Similar to previous results, use of the P3-GFP reporter system indicated induction of $a g r$ signaling in unbuffered medium (Figure 3-3B). However, when the $\mathrm{pH}$ was buffered to acidic conditions ( $\mathrm{pH}$ 5.5), agr signaling was significantly attenuated (Figure 3-3B). When the $\mathrm{pH}$ was set to the agr optimum ( $\mathrm{pH}$ 6.5), much higher levels of agr signaling were observed; however, differences between mono and co-culture were no longer distinguishable (Figure 3-3B). Similar results were found at a slightly more alkaline $\mathrm{pH}$ (7.5), where $a g r$ signaling between mono and co-cultures was indiscernible (Figure 3-3B). These results suggest that extracellular $\mathrm{pH}$ strongly influences agr signaling in vitro and likely drives augmentation of toxin production during co-culture.

In order to rule out the possibility that growth rates between different $\mathrm{pH}$ conditions were impacting agr reporter results, aliquots of each culture were taken at the experimental endpoint and plated onto selective microbiological media to enumerate both C. albicans and $S$. aureus. Although there was slight variation between each condition, there was no significant difference in the number of colonies of $C$. albicans

(Figure 3-3C, blue) recovered during mono- (solid) and co-culture (hashed). Moreover, there was no significant difference between growth in buffered or unbuffered media. The same finding was true for $S$. aureus (Figure 3-3C, yellow) during mono (solid) and coculture (hashed). Collectively, these results suggest that extracellular $\mathrm{pH}$ and not microbial growth accounts for disparate agr activity observed during mono and coculture.

In order to rule out the possibility that a factor secreted by $C$. albicans activates agr signaling, $S$. aureus(pDB22) was grown at $\mathrm{pH} 5.5$ overnight to attenuate $a g r$ signaling. Spent culture supernatants from C. albicans or S. aureus (JE2) grown at $\mathrm{pH} 7$ were filter sterilized, reconstituted in fresh concentrated growth medium, and added to $S$. aureus(pDB22) to elicit agr activation. Fresh culture medium was also used as a control. Results demonstrated that while spent culture medium from $S$. aureus (JE2) more rapidly and robustly activated the agr system, addition of spent $C$. albicans supernatant did not differ from the fresh culture medium, suggesting that culture $\mathrm{pH}$ and not a specific candidal factor drives quorum signaling (Figure 3-3D).

Several recent reports have demonstrated that $C$. albicans possesses the incredible capacity to rapidly alkalinize its external environment via amino acid catabolism (124, $126,127)$. This process is primarily driven during carbohydrate stress by sensing of amino acids in the milieu and regulating amino acid import, largely governed by the transcription factor Stp2p. Ammonia is extruded from the cell as these peptide substrates 

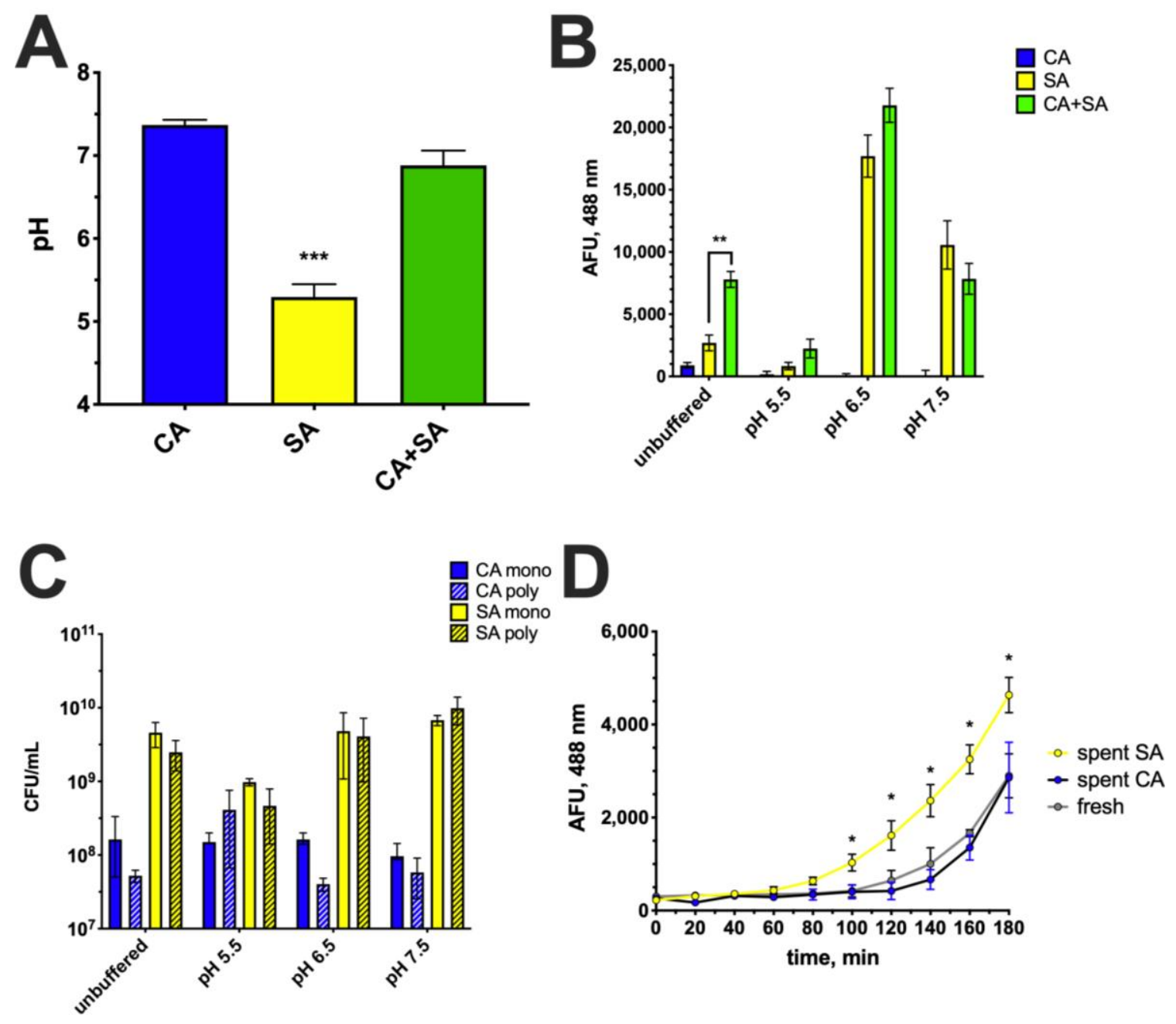

Figure 3-3. Synergistic hemolysis is partially dependent on extracellular pH and can be overridden by its modulation.

(A) The $\mathrm{pH}$ of monomicrobial (CA or SA) and polymicrobial (CA+SA) cultures was measured after $16 \mathrm{~h}$ incubation at $37^{\circ} \mathrm{C}$. (B) Assays were conducted similarly as in panel $\mathrm{A}$, except that the $\mathrm{pH}$ of TSB-g was buffered as indicated. Activation of staphylococcal $a g r$ was assessed using a GFP-reporter assay. (C) Aliquots from each culture were taken at the endpoint to measure microbial counts by plating on selective media. Blue bars indicate $C$. albicans counts and yellow bars indicate $S$. aureus counts. Solid bars depict counts from monomicrobial (mono) cultures, while hashed bars depict counts from polymicrobial (poly) cultures. (D) Buffered (pH 7.0) spent CA and SA culture supernatants or fresh media were added to $S$. aureus(pDB22) initially grown at $\mathrm{pH} 5.5$. Fluorescence $(488 \mathrm{~nm})$ was captured kinetically to assess agr activation. All data is representative of 3 independent repeats and expressed as the mean \pm SEM. Data was assessed for significance using one-way ANOVA and Dunnett's posttest. ${ }^{* *}, P<0.01$; ***, $P<0.001$. 
are consumed by the fungus, ultimately raising the extracellular $\mathrm{pH}(68,128)$. Therefore, we utilized an stp $2 \Delta / \Delta$ mutant and isogenic revertant strain (stp $2 \Delta / \Delta+S T P 2)$ to determine the impact of alkalinization during co-culture with $S$. aureus. Culture $\mathrm{pH}$ of the $\operatorname{stp} 2 \Delta / \Delta$ during co-culture was significantly decreased as compared to that of wild-type or revertant strains (Figure 3-4A). In a similar fashion, production of $\alpha$-toxin was significantly attenuated during co-culture with the $\operatorname{stp} 2 \Delta / \Delta$ strain (Figure 3-4B). Thus, active alkalinization of the external environment by $C$. albicans partially modulates staphylococcal $\alpha$-toxin production during co-culture.

\section{Candida Species Differentially Modulate $\alpha$-Toxin Production During Coculture}

We next questioned whether augmented $\alpha$-toxin production was specific to $C$. albicans or if other non-albicans Candida (NAC) species could also potentiate this effect. Therefore, $S$. aureus was cultivated by itself or in the presence of various Candida species, including $C$. albicans, $C$. glabrata, $C$. dubliniensis, $C$. tropicalis, $C$. parapsilosis, and $C$. krusei. Measurement of the extracellular $\mathrm{pH}$ following co-culture with $C$. albicans, $C$. tropicalis, and $C$. krusei revealed significantly increased neutralization over $S$. aureus monoculture (Figure 3-5A). Co-culture with $C$. glabrata, $C$. dubliniensis, and C. parapsilosis demonstrated only modest increases in $\mathrm{pH}$ which were generally below the threshold for robust agr activation. These results were recapitulated by examining $\alpha-$ toxin production during co-culture. Candida species capable of significantly raising the extracellular $\mathrm{pH}$ in this assay ( $C$. albicans, $C$. tropicalis, $C$. krusei) also demonstrated increased capacity to augment $\alpha$-toxin release, while those incapable of significantly modulating the extracellular $\mathrm{pH}$ demonstrated only modest elevation of this virulence determinant (Figure 3-5B). These results demonstrate that exacerbation of $\alpha$-toxin production is not limited to $C$. albicans, but this in vitro phenotype is largely driven by modulation of extracellular $\mathrm{pH}$ during co-culture.

\section{Conclusions}

Often, the pathogenic process is delineated as the interaction between host and microbe resulting in some level of host detriment. Unfortunately, the contribution of microenvironment is all too frequently disregarded in this description. However, changes in environmental factors can ultimately drive or control pathogenicity or host susceptibility. Through the prism of single microbe infections, this concept seems obvious. For example, gene expression exhibited by $C$. albicans is very different in the gut as compared to in the oral cavity-largely attributable to variations in host cell type, oxygen tension, nutrient availability, and microbial competition $(7,68,129)$. In the context of a polymicrobial model of disease, gene regulation in response to environment by one organism may elicit reactions by a second pathogen, opportunist, or colonizer. An excellent example of this is the capacity of the lactobacilli to ferment carbohydrates to lactic acid at the vaginal mucosa (130). Production of relatively high levels of lactic acid keeps the vaginal $\mathrm{pH}$ low and consequently impairs the overgrowth of $C$. albicans, limiting the development of vulvovaginal candidiasis. While there are several examples 

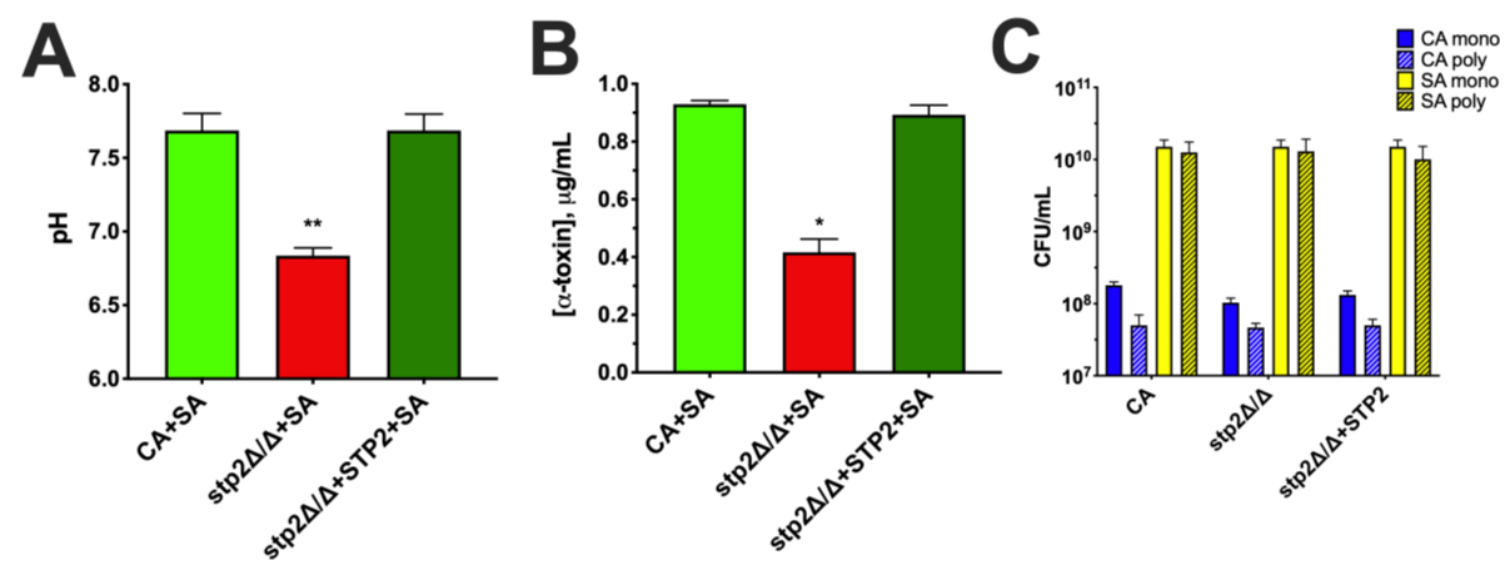

Figure 3-4. The alkalinization-deficient $C$. albicans mutant $\operatorname{stp} 2 \Delta / \Delta$ fails to enhance $S$. aureus toxin production.

Polymicrobial cultures of $S$. aureus and wild-type $C$. albicans $(\mathrm{CA}+\mathrm{SA}), \mathrm{SA}$ and STP2 mutant $(\operatorname{stp} 2 \Delta / \Delta+\mathrm{SA})$, or SA and $S T P 2$ revertant $(\operatorname{stp} 2 \Delta / \Delta+S T P 2+\mathrm{SA})$ were incubated at $37^{\circ} \mathrm{C}$ in media initially set to $\mathrm{pH} 6$. At $24 \mathrm{~h}$, (A) the $\mathrm{pH}$ of the cultures was measured using a $\mathrm{pH}$ meter and (B) $\alpha$-toxin levels in supernatants were determined by ELISA. (C) Aliquots from each culture were taken at the endpoint to measure microbial counts by plating on selective media. Blue bars indicate $C$. albicans counts, and yellow bars indicate $S$. aureus counts. Solid color bars depict counts from monomicrobial (mono) cultures, while hashed bars depict counts from polymicrobial (poly) cultures. Data are representative of 3 independent repeats and expressed as the mean \pm SEM. Data was assessed for significance using one-way ANOVA and Dunnett's posttest. *, $P<0.05$; **, $P<0.01$. 

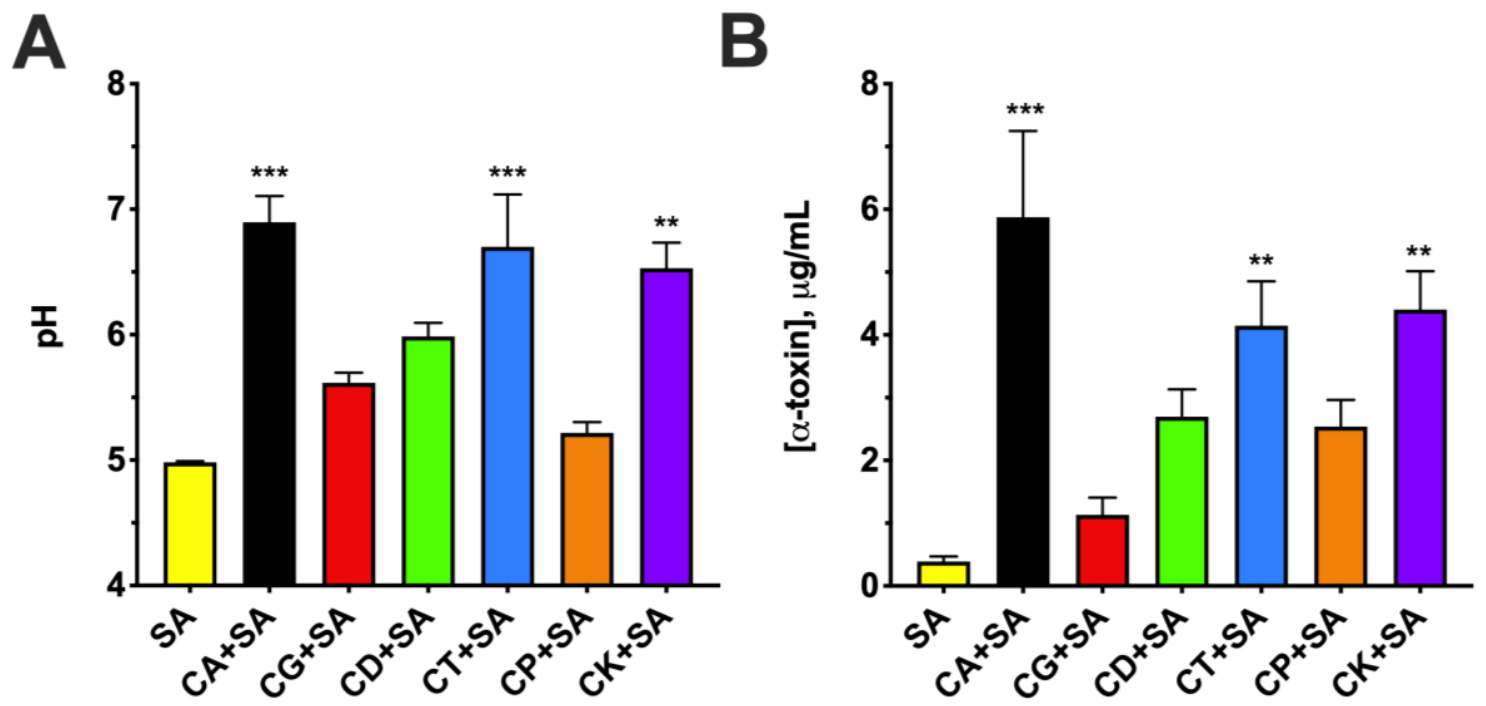

Figure 3-5. Candida spp. differ in their capacity to modulate extracellular pH and augment toxin production during polymicrobial growth with $S$. aureus.

Polymicrobial cultures of Candida species [C. albicans (CA), C. glabrata (CG), $C$.

dubliniensis (CD), C. tropicalis (CT), C. parapsilosis $(\mathrm{CP})$, C. krusei $(\mathrm{CK})]$ and S. aureus (SA) were incubated at $37^{\circ} \mathrm{C}$. After 16 hours of growth, (A) the $\mathrm{pH}$ of the cultures was measured using a standard $\mathrm{pH}$ meter and (B) the level of $\alpha$-toxin in the supernatant was assessed by ELISA. Data is representative of 5 independent repeats and expressed as the mean \pm SEM. Data was assessed for significance using one-way ANOVA and Dunnett's posttest. **, $P<0.01$; ***, $P<0.001$. 
of "environmental crosstalk" resulting in microbial antagonism, reports regarding exacerbated virulence are less common $(8,17,19,20)$.

We, along with others, have previously shown that murine intra-abdominal coinfection with $C$. albicans and $S$. aureus results in a strikingly high mortality rate ( $90 \%)$ within $\sim 16 \mathrm{~h}$ p.i., while infection with either microbe alone is non-lethal (65). Moreover, this synergistic lethality is dependent on $\alpha$-toxin, as an isogenic strain lacking the gene encoding for this virulence factor $(h l a)$ or neutralization of this toxin using a high affinity antibody resulted in significant protection. In support of this, lavage of the peritoneal cavity following infection revealed $\sim 4$-fold higher levels of $\alpha$-toxin in co-infected mice as compared to those challenged with $S$. aureus alone (65). These in vivo results substantially mimic those observed in the in vitro system used in this study. Using several functional assays, we confirmed that $\alpha$-toxin levels are significantly elevated during polymicrobial as compared to monomicrobial growth in an agr-dependent fashion. Interestingly, the mechanism of $\alpha$-toxin enhancement was not likely due to direct fungal ligand binding or recognition by the agr quorum sensing system, as $S$. aureus mutants with disrupted $a g r$ genes ( $\triangle a g r A$ or $\triangle a g r B$ ) failed to demonstrate toxin production even during co-culture with $C$. albicans, indicating necessity of an intact agr regulon (Figure 3-2). There are numerous examples where secreted microbial peptides or metabolites can exhibit a diverse array of effects on neighboring cells of the same or different species (17, $19,131)$. Deletion of $\operatorname{agr} B$, the auto-inducing peptide permease, should still allow for functional agr signaling in the context of candidal factors capable of inducing the AgrCAgrA two-component system. Observed failure to activate toxigenic and agr responses under such conditions or with spent $C$. albicans culture supernatant likely indicates that potential fungal ligands do not directly signal via the AgrC surface receptor.

The exclusion of a factor directly engaging agr led us to investigate potential indirect influences, such as modulation of environmental factors. In pioneering work characterizing the agr quorum sensing system in S. aureus, Regassa et al. demonstrated the $\mathrm{pH}$-responsiveness of the agr system. They reported increased agr activity during growth at $\mathrm{pH} 6.5-7$ as compared to growth in more alkaline or acidic media $(115,123)$. The $\mathrm{pH}$ of monomicrobial $S$. aureus cultures demonstrated significant acidification $(\mathrm{pH}$ 5.0-5.5), while co-cultures exhibited a $\mathrm{pH}$ of 6.5-7, exactly in the range of maximum agr activity (Figure 3-3A). Buffering of the media demonstrated that agr signaling could be manipulated positively or negatively during co-culture depending on $\mathrm{pH}$ selection, further demonstrating the importance of environmental factors (e.g. $\mathrm{pH}$ ) in driving pathogenicity mechanisms in vitro. Both $C$. albicans and $S$. aureus preferentially utilize glucose to undergo oxidative and fermentative metabolism, ultimately producing acidic endproducts that drive lower culture $\mathrm{pH}$. So then why does the culture medium containing $C$. albicans demonstrate an elevated $\mathrm{pH}$ ? The ability of $C$. albicans (and other fungal species) to alkalinize its environment has been well documented in the literature. Vylkova et al. have demonstrated that $C$. albicans can raise the $\mathrm{pH}$ of macrophage phagosomes, allowing for hyphal growth that damages the phagosomal membrane, aiding in escape and continued proliferation $(124,126)$. Moreover, the acidic $\mathrm{pH}(\leq 5)$ of the phagosome is vital for the activity of degradative enzymes that act to kill and digest engulfed pathogens, including fungi. Additionally, low $\mathrm{pH}$ is known to repress the 
morphological transition of yeast-to-hyphae, the major virulence attribute of $C$. albicans (33). Therefore, $C$. albicans has evolved strategies to modulate the external $\mathrm{pH}$ to likely bypass host checkpoints and killing mechanisms.

We propose that during polymicrobial growth, the $\mathrm{pH}$ initially decreases as glucose is metabolized by both organisms, then when glucose is limiting, the culture medium is alkalinized by $C$. albicans, in turn activating the agr quorum sensing system and subsequent $\alpha$-toxin production. The mechanism of alkalinization involves the breakdown of amino acids for a carbon source and the subsequent excretion of ammonia, which raises the $\mathrm{pH}$. In glucose-limited environments, amino acids are sensed by the SPS sensor system, a complex of three proteins (Ssy1, Ptr3, and Ssy5). This sensor complex induces the proteolytic cleavage of a cytoplasmic retention signal of Stp2p, allowing it to translocate to the nucleus. Stp2p binds SPS sensor-regulated promoters of various amino acid permease genes, including $C A N 1, G A P 1$, and $G A P 2$, which transport extracellular amino acids into the cell (132). Amino acid catabolism begins with the deamination of an amino acid, catalyzed by amino acid-specific deaminases. The carbon backbone is converted to TCA cycle intermediates (pyruvate, $\alpha$-ketoglutarate, acetoacetyl-CoA) via the production of acetyl-CoA. The nitrogen is converted to ammonia and $\mathrm{CO}_{2}$ by the urea amidolyase Dur1,2p, and subsequently excreted from the cell through various ATO (ammonia transport outward) family transmembrane proteins $(127,128,132)$. C. albicans strains lacking STP2 have an impaired capacity to alkalinize the environment compared to isogenic controls, corresponding with a decrease in ammonia produced during growth. Additionally, this mutant is unable to form hyphae after phagocytosis, preventing escape from the phagosome, which also leads to more effective killing by the macrophage. In a mouse model of disseminated candidiasis, stp $2 \Delta / \Delta$ mutants displayed attenuated virulence, as mortality was significantly delayed compared to wild-type or complemented strains (124). Using a $\operatorname{stp} 2 \Delta / \Delta$ strain in our in vitro polymicrobial culture system, we found that the mutant cannot raise the $\mathrm{pH}$ to levels observed with wild-type or the revertant strains. This further confirms that the capacity of $C$. albicans to alkalinize the media contributes to enhanced $\alpha$-toxin production during co-culture (Figure 3-4).

Although Stp2p is largely responsible for driving the alkalinization phenotype in C. albicans, it is not the sole mechanism for $\mathrm{pH}$ modulation. A number of C. albicans genes have been identified as having effects on external alkalinization, including ALI1, SIN3, COX4, PEP8, KIS1, and CPH1. Some of these genes (COX4 and KIS1) are linked to carbon metabolism, while $C P H 1$ can regulate galactose utilization. $C$. albicans has also been shown to modulate $\mathrm{pH}$ without the production and excretion of ammonia, as seen during growth with non-fermentable carbon sources, like the carboxylic acids $\alpha$ ketoglutarate, pyruvate, and lactate $(128,132)$. Interestingly, alkalinization occurs in low glucose environments, regardless of the mechanism, indicating that this effect is glucose repressible. Vylkova et al. hypothesize that alkalinization of the phagosome is due to limited glucose within the phagosomal compartment (124). Although glucose or other metabolic compounds were not a direct focus of this paper, their potential effect on $\mathrm{pH}$ and $\alpha$-toxin production can be inferred. In addition to being responsive to $\mathrm{pH}$, the $a g r$ quorum sensing system is known to be regulated by glucose levels, with growth under high glucose conditions correlated to low agr activity. Future studies focusing on the 
metabolic profiles of both $C$. albicans and $S$. aureus may shed more light on this potential effector mechanism.

Lastly, we investigated the ability of other NAC species to enhance $\alpha$-toxin production during in vitro growth. We found that the species differ in their alkalinization potential, with only $C$. tropicalis and $C$. krusei able to raise the $\mathrm{pH}$ of the media to levels similar to that of $C$. albicans during polymicrobial growth with $S$. aureus. Additionally, these three species were the only ones that caused a significant increase in the amount of $\alpha$-toxin produced, compared to $S$. aureus monomicrobial culture (Figure 3-5). These data correlate nicely with work performed by Nash et al. in which mice were co-infected with the exact same Candida strains. Mortality was observed in mice infected with $S$. aureus and $C$. albicans, $C$. tropicalis, and $C$. krusei. Strikingly, the species unable to modulate the $\mathrm{pH}$ and $\alpha$-toxin production during in vitro growth in this study $(C$. glabrata, $C$. dubliniensis, and $C$. parapsilosis) were also non-lethal in the polymicrobial IAI model (67). A retrospective analysis of intra-abdominal infections, specifically looking at candidiasis, revealed that $C$. albicans is the most commonly isolated species (50-75\%). The next most commonly isolated species is $C$. glabrata (12-25\%), followed by $C$. parapsilosis (3-10\%), C. tropicalis (3-5\%), and C. krusei (3-6\%) (133, 134). Interestingly, $C$. glabrata was implicated as the infectious organism in the majority (64\%) of recurrent or persistent infections (133). Cheng et al. described persistent $C$. glabrata IAI in mice and an association with the formation of abscesses (68).

Various niches within the body are maintained at drastically different $\mathrm{pH}$ values. For example, the oral cavity is maintained at $\mathrm{pH} 6.2-7.6$ by saliva (135). The blood is very tightly buffered to remain at $\mathrm{pH} 7.4$, and a decrease in 0.05 units causes severe physiological problems, as seen in diabetic ketoacidosis (136). The vagina is more acidic, with a healthy $\mathrm{pH}$ ranging from $3.8-4.5$ (137). The fluid within the peritoneal cavity of humans is reported to be at a $\mathrm{pH}$ of 7.5 - 8 (138). Although widely variable, bodily $\mathrm{pH}$ is tightly regulated to maintain homeostasis and dysregulation is often indicative of poor health. A study investigating the transcriptome of $C$. albicans during murine IAI found that a number of genes involved in $\mathrm{pH}$ response were among the most highly upregulated. These genes include RIM101, the alkaline $\mathrm{pH}$-regulated transcription factor that modulates morphology and gene expression (68). Indeed, a rim101 $\Delta / \Delta$ strain exhibited attenuated virulence during peritoneal infection, characterized by significantly lower fungal burdens (68). These findings indicate that the murine peritoneal cavity is alkaline and that $C$. albicans requires adaptation to $\mathrm{pH}$ to establish pathogenicity in this biological niche. However, because of the homeostatic nature of $\mathrm{pH}$ maintenance, it is unlikely that that $C$. albicans is able to drastically alter the global $\mathrm{pH}$ of the peritoneal cavity during IAI. Our attempts to experimentally monitor broad $\mathrm{pH}$ changes in the peritoneal lavage fluid during mono- or co-infection have demonstrated insignificant differences, partially due to sensitivity of the techniques utilized (e.g. phenol red lavage, micro $\mathrm{pH}$ electrode) or spatiotemporal kinetics. Thus, it is more likely that microenvironmental $\mathrm{pH}$ regulation, in peritoneal abscesses or tissues, may play an important role in driving these phenotypes. As $C$. albicans and $S$. aureus are known to tightly associate via staphylococcal binding of fungal hyphae via the candidal adhesin Als3p (50), it is possible that elevated S. aureus 
$\alpha$-toxin production in vivo is due to local $\mathrm{pH}$ changes surrounding fungal-bacterial aggregates. However, this hypothesis requires future investigation.

Collectively, results from this study highlight the dynamic and complex nature of this fascinating microbial pair and polymicrobial interactions in general. Furthermore, they underscore the importance of environmental adaptation and its intersection with virulence that must be considered in the context of co-infection. 


\section{CHAPTER 4. A CRITICAL ROLE FOR THE CANDIDA ALBICANS TRANSCRIPTION FACTOR, ZCF13, IN ENHANCING STAPHYLOCOCCUS AUREUS $\alpha$-TOXIN PRODUCTION}

\section{Introduction}

Candida albicans, an opportunistic fungus, and Staphylococcus aureus, a ubiquitous bacterial pathogen, are among the top causes of serious nosocomial infections and invasive diseases $(73,74)$. While these microbes are able to cause significant morbidity and mortality on their own, they are often co-isolated from various niches and are correlated with more severe disease states and higher mortality rates, even with therapeutic intervention $(42,44,56,57,120,139)$. Intra-abdominal infections (IAI), which are a collection of diseases characterized by microbial invasion into and inflammation of the abdominal cavity, are often polymicrobial (133). The introduction of microbes generally results from trauma, such as perforations to the gastrointestinal tract, invasive surgery, and contamination of indwelling catheters. IAI can lead to more complicated infections, like sepsis, and are the second-most common cause of infectious mortality in ICU patients (55). Fungal-bacterial polymicrobial IAI result in more severe disease and increased mortality, up to $80 \%$, compared to $10-30 \%$ mortality in bacterial IAI $(56,57)$. Additionally, $C$. albicans has been identified as an independent risk factor for mortality during IAI (140).

In support of this clinical data, a mouse model of polymicrobial IAI using $C$. albicans and $S$. aureus revealed a striking lethal synergism, where co-inoculation rapidly resulted in $100 \%$ mortality, while monomicrobial infections were non-lethal (59). $S$. aureus produces a number of toxins, primarily regulated through the agr quorum sensing system (32). Previous work from our laboratory has demonstrated that C. albicans is able to augment $S$. aureus agr activity and upregulate agr-associated genes during polymicrobial growth, most notably that responsible for $\alpha$-toxin production (65). Encoded by the hla gene, $\alpha$-toxin is a small hemolytic exotoxin that is secreted as a monomer which oligomerizes into a heptameric $\beta$-barrel and is capable of nonspecifically forming pores in many different cell types, including red blood cells, epithelial cells, endothelial cells, and various immune cells. It is also recognized by a disintegrin and metalloproteinase domain-containing protein 10 (ADAM10) receptor in the membrane with high affinity (30). Upon binding to ADAM10, $\alpha$-toxin inserts its stem domain into the membrane, forming a pore through which cations and low molecular weight molecules can flow, leading to cell lysis. However, $\alpha$-toxin can also interact with cells in a receptor-independent manner at high concentrations by binding to host membrane lipids, thus causing lysis without specific binding to ADAM-10 (141). Subsequent cytolysis disrupts endothelial junctions and initiates pro-inflammatory immune responses by activating multiple signaling cascades, including the NLRP3 inflammasome (30). We have previously shown that inflammatory markers, such prostaglandin PGE $_{2}$ and the proinflammatory cytokines IL-6, IL-1 $\beta$, and TNF- $\alpha$, are significantly upregulated in the peritoneum of C. albicans-S. aureus infected mice (62, $65,66)$. In addition, $\alpha$-toxin mediates platelet aggregation, leading to excessive 
microvascular clotting and thrombocytopenia observed in staphylococcal sepsis. Dysregulation of thrombosis can lead to significant host organ damage that contributes to the pathogenesis of sepsis. $\alpha$-toxin has been shown to directly damage both the liver and kidneys through the induction of thrombotic events $(58,142)$.

While it has been established that $C$. albicans is able to enhance staphylococcal $\alpha$ toxin production in vitro and in vivo, the mechanism(s) by which $C$. albicans achieves this are incompletely defined (65). We have previously determined that there is not likely a candidal secreted factor that is directly stimulating the AgrC receptor of $S$. aureus to enhance $\alpha$-toxin production (122). However, we found that extracellular alkalinization by $C$. albicans plays an important role in modulating agr signaling. The $a g r$ system is responsive to $\mathrm{pH}$, and during co-culture $C$. albicans raises the $\mathrm{pH}$ to optimal levels for agr activity. Additionally, an alkalinization-deficient mutant $(\Delta / \Delta s t p 2)$ is unable to enhance $a g r$ activity and $\alpha$-toxin production in vitro (122). However, this mutant showed no pathogenicity defect during co-infection in the mouse model of IAI, and additional genetically encoded or physiological stimuli must be required for synergistic lethality (Figure 4-1).

Thus, the objective of this study was to further clarify the mechanism driving synergistic lethality during $C$. albicans-S. aureus polymicrobial IAI by identifying novel regulators of Candida-induced agr activation. In support of this, we show that staphylococcal $\alpha$-toxin is necessary for exacerbated damage to the liver and kidneys during co-infection and that an uncharacterized candidal transcription factor, ZCF 13, is required for augmenting these effector responses and $\alpha$-toxin production.

\section{Materials and Methods}

\section{Ethics Statement}

The animals used in this study were housed in AAALAC-approved facilities located at the University of Tennessee Health Sciences Center (UTHSC) in the Regional Biocontainment Laboratory (RBL). The UTHSC Animal Care and Use Committee, Laboratory Animal Care Unit (LACU) approved all animal usage and protocols (protocol \#18-060). Mice were given standard rodent chow and water ad libitum. Mice were monitored daily for signs of distress, including noticeable weight loss and lethargy. UTHSC LACU uses the Public Health Policy on Humane Care and Use of Laboratory Animals (PHS) and the Guide for the Care and Use of Laboratory Animals as a basis for establishing and maintaining an institutional program for activities involving animals. To ensure high standards for animal welfare, UTHSC LACU remains compliant with all applicable provisions of the Animal Welfare Act (AWAR), guidance from the Office of Laboratory Animal Welfare (OLAW), and the American Veterinary Medical Association Guidelines on Euthanasia. 

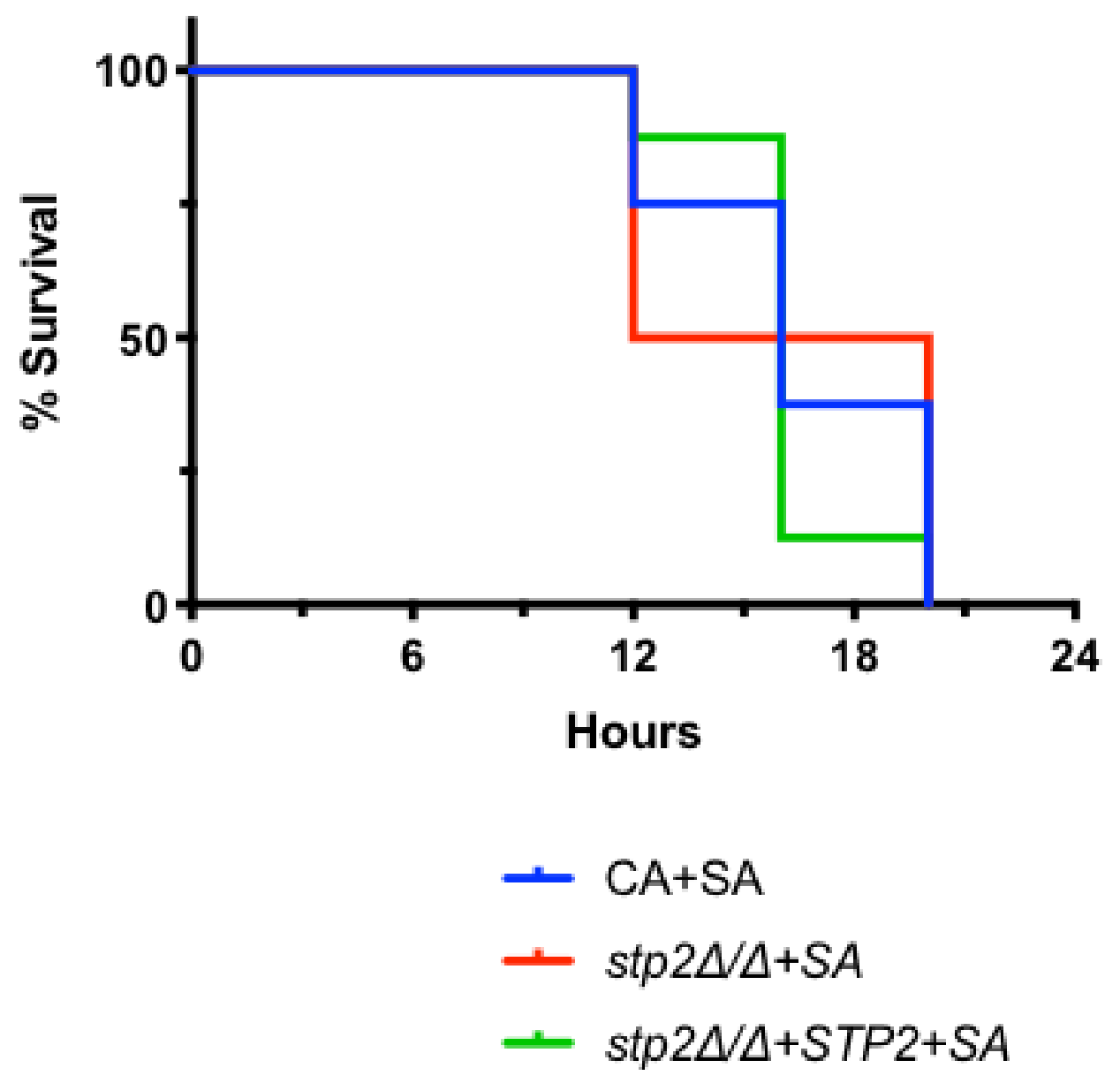

Figure 4-1. An alkalinization-deficient mutant shows no defect in synergistic lethality in polymicrobial IAI.

Mice ( $\mathrm{n}=8$ per group) were infected with SA and either $C$. albicans SC5314, $\operatorname{stp} 2 \Delta / \Delta$, or stp $2 \Delta / \Delta+S T P 2$ and followed for survival. Experiments were repeated twice and data combined. Significance was assessed using a Wilcoxon log rank test. 


\section{Strains and Growth Conditions}

Candida albicans strain SC5314 (CA) was used as the wild-type/reference strain for all experiments unless otherwise noted. The alkalinization-deficient mutant $\operatorname{stp} 2 \Delta / \Delta$ (SVC17) and its isogenic revertant strain STP2-REV (SVC19) were kind gifts from Dr. Michael Lorenz (University of Texas Health Science Center) (124). A library of $C$. albicans transcription factor deletion mutants and accompanying wild-type background strain (TF WT) were obtained from the Fungal Genetics Stock Center (143). Strains were maintained in glycerol stocks in 96 -well plates and stored at $-80^{\circ} \mathrm{C}$. S. aureus strain JE2 (SA) (a USA300 isolate used as wild-type) and strain NE1354 ( $\Delta h l a)$ ( $\alpha$-toxin-deficient) were obtained from the Biodefence and Emerging Infectious (BEI) Research Resources repository (89). A $S$. aureus reporter strain [S. aureus(pDB22)] (containing plasmid pDB22 with the P3 promoter fused to GFP mut2 and erythromycin resistance cassette) was also used in this work, as described previously (125).

Candida strains were streaked onto yeast peptone dextrose (YPD) agar plates and grown at $30^{\circ} \mathrm{C}$. Single colonies were inoculated into $1.5 \mathrm{~mL}$ YPD broth and grown at $30^{\circ} \mathrm{C}$ with shaking at $200 \mathrm{rpm}$. S. aureus strains were streaked onto trypticase soy agar (TSA) (with antibiotic added as needed) and grown at $37^{\circ} \mathrm{C}$. Single colonies were inoculated into $1.5 \mathrm{~mL}$ TSB (with antibiotic added as needed) and grown at $37^{\circ} \mathrm{C}$ with shaking at $200 \mathrm{rpm}$. Escherichia coli strains DH5- $\alpha$ and IM08B (obtained through BEI Resources) were used for plasmid construction and were grown on Luria-Bertani (LB) agar supplemented with $100 \mu \mathrm{g} / \mathrm{mL}$ ampicillin or $50 \mu \mathrm{g} / \mathrm{mL}$ kanamycin as described (91, 144).

\section{Murine Model of IAI}

I.p. inoculations were conducted as described previously $(62,65-67)$. Groups $(\mathrm{n}=4)$ of six-to-eight-week old Swiss Webster mice were injected intraperitoneally (i.p.) using a 27 -gauge $1 / 2$ " needle with $1.75 \times 10^{7}$ or $7 \times 10^{6} \mathrm{CFU}$ of $C$. albicans, $8 \times 10^{7} \mathrm{CFU}$ of $S$. aureus, or the above doses of each microbe simultaneously. Inocula were prepared in a final volume of $0.2 \mathrm{~mL}$ pyrogen-free phosphate buffered saline (PBS). After inoculation mice were observed up to $5 \mathrm{~d}$ p.i. for morbidity (hunched posture, inactivity, ruffled fur) and mortality. Mice that exhibited severe morbidity were humanely sacrificed and tallied as a lethal outcome.

In some experiments, mice were sacrificed $8 \mathrm{~h}$ p.i. prior to severe morbidity. Peritoneal cavities were lavaged by injection of $2 \mathrm{~mL}$ of sterile PBS containing 1X protease inhibitors (cOmplete, Roche) followed by gentle massaging of the peritoneal cavity. Peritoneal lavage fluid was then removed using a pipette inserted into a small incision in the abdominal cavity. Both kidneys and the spleen were removed from infected mice and placed in $500 \mu \mathrm{L}$ PBS for homogenization prior to CFU enumeration and ELISA analysis. Whole blood was collected by cardiac puncture and serum was separated by centrifugation. Animal experiments were repeated in duplicate and results combined. 


\section{Clinical Chemistry Analysis}

Clinical chemistry analysis of serum was performed using a DiaSys Respons ${ }^{\circledR}$ 910Vet chemistry analyzer (DiaSys Diagnostic Systems, USA. Wixon, MI). All tests were calibrated (TruCalU calibrator, DiaSys Diagnostic System) and bi-level quality control materials (TruLab N and TruLab P, DiaSys Diagnostic Systems) run prior to sample analysis. The Respons 910Vet chemistry analyzer uses colorimetry with either a rate or end point reaction method. All reagents were purchased from DiaSys and analysis performed by RBL staff according to manufacturer's established procedures.

\section{Site-Directed Mutagenesis}

Construction of the oligomerization-deficient H35L $\alpha$-toxin mutant was done using Platinum SuperFi I (ThermoFisher) following the manufacturer's site-directed mutagenesis protocol. Primers H35L-Superfi-F and H35L-Superfi-R were used to PCR amplify pSK-hla (vector pSK5630 containing the entire hla ORF from S. aureus JE2 (Table 4-1) (122). Amplified DNA was digested with DpnI to remove residual template DNA and transformed into the $d \mathrm{~cm}$-deficient $E$. coli strain IM08B to yield plasmid pSKH35L. pSK-H35L was electroporated into $S$. aureus NE1345 $\Delta$ hla and selected on TSA with $10 \mu \mathrm{g} / \mathrm{mL}$ chloramphenicol to create strain $\Delta h l a-\mathrm{p} h l a-\mathrm{H} 35 \mathrm{~L}$. To confirm correct mutagenesis, plasmid isolated from colonies underwent Sanger sequencing using primers hlaSeqF and hlaSeqR.

\section{Construction of Luciferase Reporter}

To construct a P3-luciferase reporter plasmid, the $S$. aureus $\mathrm{P} 3$ promoter was PCR amplified from a gBlock containing the $\mathrm{P} 3$ promoter and flanking amplification and restriction sequences (Table 4-2). This PCR product and plasmid pMV306G13+Lux (Addgene plasmid \# 26160 ; http://n2t.net/addgene:26160 ; RRID:Addgene_26160) containing the LuxABCDE operon were digested with NotI and NcoI, ligated, and transformed into E.coli DH5- $\alpha$ to yield pOLux1 using the method previously described (65). The entire P3-LuxABCDE operon from pOLux1 was PCR amplified using primers luxABCDE-Fv2-EcoRI and luxABCDE-R-NarI. This product, along with plasmid pMK4, were digested with EcoRI and NarI. Digests were ligated and transformed into E.coli DH5- $\alpha$, yielding plasmid pOLux2. The constitutive S. aureus promoter sarA P1 was synthesized as a gBlock and, along with pOLux2, was digested with Acc65I, ligated, and transformed into E.coli DH5- $\alpha$, yielding pOLux3. Correct orientation of the sarA P1 promoter in pOLux3 was confirmed by PCR amplifying with primers sarAP1-FAcc65I and luxC-DET-R. pOLux 3 was transformed into $E$. coli IMO8B, as previously described (65). After confirmatory digestion, plasmids were transformed into S. aureus JE2 competent cells and plated on brain heart infusion agar containing $10 \mu \mathrm{g} / \mathrm{mL}$ chloramphenicol to yield strain $S$. aureus(pOLux). The P3 promoter drives expression of the $P$. luminescens luciferase enzyme in an agr-dependent manner, and the constitutively active sarA P1 promoter allows for constitutive expression of the substrate luciferin. 
Table 4-1. Oligonucleotides used in this work.

\begin{tabular}{|c|c|}
\hline Primer Name & Sequence $5^{\prime} \rightarrow 3^{\prime}$ \\
\hline H35L-Superfi-F & ATGGCATGCTCAAAAAAGTATTTTATAG \\
\hline H35L-Superfi-R & TACTTTTTTGAGCATGCCATTTTCTTTATC \\
\hline hlaSeqF & GATATGTCTCAACTGCAATATTCTAAATTGACATA \\
\hline hlaSeqR & ACATCATTTCTGAAGTTATCGGC \\
\hline P3lux-F-NotI & $\begin{array}{l}\text { TCAGCGGCCGCATTTTAACATAAAAAAATTTACAGTTAA } \\
\text { GAATAAAAAACGACTAG }\end{array}$ \\
\hline P3Lux-R-NcoI & $\begin{array}{l}\text { TCACCATGGGATCTCTGTAATCTAGTTATATTAAAACAT } \\
\text { GCTAAAAGCAT }\end{array}$ \\
\hline luxABCDE-Fv2- & TCAGAATTCATTTTAACATAAAAAAATTTACAGTTAAGA \\
\hline EcoRI & ATAAAAAACGACTAG \\
\hline $\begin{array}{l}\text { luxABCDE-R- } \\
\text { NarI }\end{array}$ & TCAGGCGCCGATCACCGCGGCCATGAT \\
\hline sarAP1-F-Acc65I & TCAGGTACCCTGATATTTTTGACTAAACCAAATGC \\
\hline sarAP1-R-Acc65I & TCAGGTACCGATGCATCTTGCTCGATACATTTG \\
\hline luxC-DET-R & GTCACGAATGTATGTCCTGC \\
\hline ZCF13-FF-KpnI & TCAGGTACCAATCAAGCCTCCTGTACCACCACCA \\
\hline ZCF13-FR-ApaI & TCAGGGCCCGCCTGGACTATTTGTCTTATCCATAATCGAT \\
\hline ZCF13-RF-NotI & TCA $\overline{\text { GCGGCCGCGTGATGAAATTTTGGATCCTGTTGCTTG }}$ \\
\hline ZCF13-RR-SacI & TCAGAGCTCGGCATGTTGTTGCTTTAGTGTCAGG \\
\hline ZCF13-AMPF- & TCACCCGGGGACATCTCTCATTTGGTATAAATGATTGTC \\
\hline SmaI & GG \\
\hline $\begin{array}{l}\text { ZCF13-AMPR- } \\
\text { NotI }\end{array}$ & TCAGCGGCCGCGCTTTAGTGTCAGGTGTTAACACAACA \\
\hline $\begin{array}{l}\text { ZCF13-FLPINT- } \\
\text { F }\end{array}$ & CAAGCCTCCTGTACCACCACCA \\
\hline $\begin{array}{l}\text { ZCF13-FLPINT- } \\
\text { R }\end{array}$ & GCTTTAGTGTCAGGTGTTAACACAACACT \\
\hline ZCF13-DET-F & CCACAACTGCAACAATCACAACAT \\
\hline ZCF13-DET-R & CGATGATGGAGCTGTTTGATCAGAT \\
\hline ZCF13-SEQ1-F & GTATCCСССТCAACTAGCAGTTAG \\
\hline ZCF13-SEQ2-F & CACCACCTTCTGTAACGACACCA \\
\hline ZCF13-SEQ3-F & GCCAATTTCACTGATGCATTTGACATGA \\
\hline ZCF13-SEQ4-F & CCGCACGTTTCAGAAGATCCC \\
\hline ZCF13-SEQ5-F & GCCGCTAGTGATCAACTGTTTTTCC \\
\hline Neut5homologyF & GCAGATATGAGATAAAAGTTTTAAAGGACAAGAAAAGG \\
\hline Nat1INTF & CCCAGATGCGAAGTTAAGTGCG \\
\hline Neut5LAMPF & GCTGAATCACTTGATAGGATTTAGTTCCATTATGG \\
\hline
\end{tabular}

Point mutations in bold and italics. Restriction sites underlined. 
Table 4-2. Sequences of gBlocks used in this work.

\begin{tabular}{ll}
\hline gBlock Name & \multicolumn{1}{c}{ Sequence 5' $\rightarrow \mathbf{3}$} \\
\hline sarA P1 & CGCAGTTACGGATCAGTCACGGTACCCTGATATTTTTGACTA \\
& AACCAAATGCTAACCCAGAAATACAATCACTGTGTCTAATG \\
& AATAATTTGTTTATAAACACTTTTTGTTTACTTCTCATTTTT \\
& AATTAGTTATAATTAACTAAATAATAGAGCATTAAATATATT \\
& TAATAAAACTTATTAATGCAAATTATGACTAACATATCTA \\
& TAATAAATAAAGATTAGATATCAATATATTATCGGGCAAATG \\
& TATCGAGCAAGATGCATCGGTACCAGCAAAGAACATGGCAG \\
& CACC \\
agr P3 & CGCAGTTACGGATCAGTCACGCGGCCGCATTTTAACATAAAA \\
& AAATTTACAGTTAAGAATAAAAAACGACTAGTTAAGAAAAA \\
& TTGGAAAATAAATGCTTTAGCATGTTTAATATAACTAGAT \\
& TCCATGGAGCAAAGAACATGGCAGCACC \\
\hline
\end{tabular}




\section{IVIS Imaging}

Groups of mice ( $\mathrm{n}=4$ ) were infected as described above, with $S$. aureus(pOLux). To minimize background interference, mice were given alfalfa-free rodent chow (Envigo) for a week prior to infection and imaging. Bioluminescence imaging was performed with Xenogen IVIS Spectrum. Mice were lightly anesthetized with isoflurane and imaged at 4 $h$ intervals (145). Images are uniformly scaled and average counts within ROI were determined with Living Image 4.7.3.

\section{GFP and Luciferase agr Reporter Assays}

Overnight cultures of $C$. albicans (YPD at $30^{\circ} \mathrm{C}$ ) and $S$. aureus(pDB22) or $S$. aureus(pOLux) (TSB at $37^{\circ} \mathrm{C}$ ) were washed three times with phosphate-buffered saline (PBS) by centrifugation. Cell concentrations were adjusted to $1 \times 10^{7} \mathrm{CFU} / \mathrm{mL}$ and 1:1000 dilutions were made into $5 \mathrm{~mL}$ of $0.6 \mathrm{X} \mathrm{TSB}+0.2 \%$ glucose $(\mathrm{TSBg})$ for monomicrobial (CA or SA) or polymicrobial $(\mathrm{CA}+\mathrm{SA})$ cultures. Antibiotic $(10 \mu \mathrm{g} / \mathrm{mL}$ erythromycin for $\mathrm{pDB} 22,10 \mu \mathrm{g} / \mathrm{mL}$ chloramphenicol for pOLux) was added for plasmid maintenance. Cultures were incubated at $37^{\circ} \mathrm{C}$ with shaking at $200 \mathrm{rpm}$ for 16 hours. 100 $\mu \mathrm{L}$ aliquots were removed in triplicate and added to wells of black (GFP) or white (luciferase) 96-well microtiter plates. Fluorescence (488 nm excitation, $525 \mathrm{~nm}$ emission) or luminescence (integration time $1 \mathrm{~min}$ ) was measured using a Synergy H1 plate reader (Biotek). Experiments were repeated in triplicate and are expressed as mean arbitrary fluorescence units (AFU) or relative light units (RLU) \pm standard error of the mean (SEM).

\section{Blood Agar Lysis Assay}

Mono- and polymicrobial cultures were prepared as above. At $16 \mathrm{~h}$ postinoculation, $5 \mathrm{~mL}$ of culture was centrifuged at 5,000 rpm and resulting supernatant was filter sterilized using a $0.2-\mu \mathrm{m}$ syringe filter. Sterile supernatants were concentrated via ethanol precipitation and resuspended in sterile water. Holes were made in blood agar plates (TSA with 5\% sheep's blood) using a sterile pipette tip. Concentrated supernatants were added to the wells and the plates were incubated at $37^{\circ} \mathrm{C}$ for $24 \mathrm{~h}$. Plates were imaged with a digital scanner (EPSON Perfection V700 Photo) and images are representative of at least three independent repeats.

\section{$\alpha$-Toxin ELISA}

The concentration of $\alpha$-toxin in culture supernatants or lavage and organ samples was measured using an $\alpha$-toxin-specific sandwich ELISA, as described previously (65, 122). Briefly, 96-well microtiter plates were coated with $50 \mu \mathrm{L} 0.1 \mu \mathrm{g} / \mathrm{mL}$ anti- $\alpha$-toxin antibody MEDI4893* diluted in ELISA/ELISPOT coating buffer (Invitrogen). Plates were incubated at $4^{\circ} \mathrm{C}$ overnight. Plates were washed with PBS-Tween 20 (PBS-T) and 
blocked with SuperBlock (Pierce) for $1 \mathrm{~h}$ at room temperature. After washing with PBS$\mathrm{T}, 50 \mu \mathrm{L}$ filter-sterilized culture supernatants, peritoneal lavage fluid, or homogenized organs were diluted in PBS, serially diluted, and plated along with native $\alpha$-toxin as the standard. Plates were incubated for $1 \mathrm{~h}$ at room temperature and then washed with PBST. Affinity-purified rabbit polyclonal anti- $\alpha$-toxin antibody $(2 \mu \mathrm{g} / \mathrm{mL})$ was added, and the plate incubated for $1 \mathrm{~h}$ at room temperature. Plates were washed with PBS-T. AffiniPure horseradish peroxidase (HRP)-coupled goat anti-rabbit IgG detection antibody (Jackson ImmunoResearch) was diluted 1:10,000 added to wells and incubated for $1 \mathrm{~h}$ at room temperature. After extensive washing with PBS-T, $100 \mu \mathrm{L}$ 3,3',5,5'-

Tetramethylbenzidine (TMB) substrate was added and plates were incubated in the dark for $10 \mathrm{~min} .100 \mu \mathrm{L}$ of $0.2 \mathrm{M} \mathrm{H}_{2} \mathrm{SO}_{4}$ was immediately added to stop the reaction. Plates were read at $450 \mathrm{~nm}$ using a plate reader (Synergy H1, BioTek). Experimental concentrations were extrapolated to the standard curve. Culture supernatants or in vivo samples from the S. aureus $\Delta$ hla strain were blank subtracted to account for any nonspecific antibody binding to shed protein A. Experiments were repeated in triplicate (in vitro) or duplicate (in vivo) and data were combined and expressed as the mean \pm SEM.

\section{CFU Enumeration}

Microbial burdens of lavage fluid, homogenized kidneys and spleens of infected mice, or culture media were determined as previously described. Briefly, serial dilutions were plated onto YPD with $20 \mu \mathrm{g} / \mathrm{mL}$ nafcillin (for $C$. albicans enumeration) and TSA with $2.5 \mu \mathrm{g} / \mathrm{mL}$ amphotericin B (for S. aureus enumeration) via the drop-plate method. Plates were incubated overnight at $37^{\circ} \mathrm{C}$. Microbial burden was enumerated and expressed as $\mathrm{CFU} / \mathrm{mL}$. CFU values are representative of at least two independent repeats and are represented as median \pm SEM.

\section{Transcription Factor Mutant Screen}

C. albicans transcription factor mutants were screened for their ability to enhance $S$. aureus agr activity following the GFP-agr reporter assay protocol. Since the background and mutant strains are arginine auxotrophs, $40 \mathrm{mg} / \mathrm{L}$ arginine was supplemented in the culture medium. Strains that were unable to significantly increase GFP expression over that of a monomicrobial $S$. aureus culture were independently confirmed and used for further analysis.

\section{Plasmid Construction}

Plasmids for ZCF13 gene disruption were constructed using plasmid pBSS2, containing the $S A T 1$-flipper disruption cassette (146). The 5' flanking region of the ZCF13 ORF was amplified from SC5314 genomic DNA using primers ZCF13-FF-KpnI and ZCF13-FR-ApaI and ligated into pBSS2 at the KpnI and ApaI restriction sites to 
create $\mathrm{pBSS} 2-\mathrm{ZCF} 13-\mathrm{F}$. The 3' flanking region of the ZCF13 ORF was amplified from SC5314 gDNA using primers ZCF13-RF-NotI and ZCF13-RR-SacI and ligated into pBSS2-ZCF13-F at the NotI and SacI restriction sites to create pBSS2-ZCF13-FR.

\section{ZCF13 Mutant Construction}

Plasmid pBSS2-ZCF13-FR was digested with KpnI and SacI and the linear fragment was transformed into SC5314 using a standard lithium acetate protocol with some modifications $(147,148)$. Overnight cultures of SC5314 were diluted 1:200 in $5 \mathrm{~mL}$ YPD and grown for $6 \mathrm{~h}$ at $30^{\circ} \mathrm{C}$ with shaking at $200 \mathrm{rpm}$ and then washed by centrifugation using sterile water. Cells were then resuspended in $1 \mathrm{X}$ TELiAc buffer (100 $\mathrm{mM}$ LiAc, $1 \mathrm{X}$ TE) and $50 \mu \mathrm{L}$ of linearized pBSS2-ZCF13-FR was added to $50 \mu \mathrm{L}$ cell suspension. Single-stranded salmon sperm carrier DNA $(5 \mu \mathrm{L})$ was added along with 300 $\mu \mathrm{L} 40 \%$ PEG. Transformation mix was incubated at $30^{\circ} \mathrm{C}$ for $30 \mathrm{~min}$ with agitation every $10 \mathrm{~min}$. Cells were heat-shocked at $42^{\circ} \mathrm{C}$ for $15 \mathrm{~min}$ then allowed to recover in YPD at $30^{\circ} \mathrm{C}$ with shaking at $200 \mathrm{rpm}$ for 4-6 h. Cells were pelleted and resuspended in $1 \mathrm{X}$ TE and plated on YPD $+200 \mu \mathrm{g} / \mathrm{mL}$ nourseothricin (YPD+200NAT). Plates were incubated for up to 2 days at $30^{\circ} \mathrm{C}$. Colonies were grown overnight in $1 \mathrm{~mL}$ YPM (YP $+2 \%$ maltose) to induce cassette excision. Cells from YPM cultures were washed with PBS then diluted to $10^{3}$ cells in $1 \mathrm{X}$ TE buffer. $100 \mu \mathrm{L}$ cells was plated onto YPD $+25 \mu \mathrm{g} / \mathrm{mL}$ nourseothricin (YPD+25NAT) and incubated at $30^{\circ} \mathrm{C}$ for $24 \mathrm{~h}$. Small and medium-sized colonies were patched onto YPD and YPD+200NAT and incubated at $30^{\circ} \mathrm{C}$ for $24 \mathrm{~h}$. Colonies that grew on YPD but not YPD+200NAT were confirmed by PCR to have ZCF13 disrupted using primers ZCF13-FLPINT-F and ZCF13-FLPINT-R, ZCF13-DET$\mathrm{F}$ and ZCF13-DET-R.

\section{ZCF13 Revertant Construction}

One copy of ZCF13 was inserted into the neutral locus NEUT5L of $\Delta / \Delta z c f 13$ using the shuttle vector pDUP3. The entire ZCF13 ORF was PCR amplified from SC5314 using primers ZCF13-AMPF-SmaI and ZCF13-AMPR-NotI. The PCR product and plasmid pDUP3 were digested with SmaI and NotI and ligated to create pDUP3ZCF13. After transformation into DH5- $\alpha$ and plasmid recovery, pDUP3-ZCF13 was linearized by digesting with SfiI and then transformed into $\Delta / \Delta z c f 13$ following the lithium acetate protocol described above. Revertant colonies were selected for by growth on YPD+200NAT and confirmed by PCR using primers Nat1INTF and Neut5LAMPF, Neut5homologyF and ZCF13-DET-R.

\section{Statistical Analyses.}

Gehan-Breslow-Wilcoxon and Wilcoxon log-rank tests were used to determine the significance of mortality. Two-tailed, unpaired two-tailed, Mann-Whitney, and oneway ANOVA and Dunnett's posttests were used to compare CFU and $\alpha$-toxin levels 
between groups, as indicated in figure legends. All graphs were constructed using GraphPad Prism version 9. Figures were composed using MS PowerPoint and rendered for publication with Adobe Photoshop.

\section{Results}

\section{$\alpha$-Toxin Is Responsible for Significant Organ Damage During Polymicrobial IAI}

Based on prior reports of organ damage caused by $\alpha$-toxin in a model of staphylococcal sepsis, we wished to determine whether $\alpha$-toxin contributed to the significant morbidity and acute mortality observed during polymicrobial IAI $(58,142)$. Mice were challenged intraperitoneally with $C$. albicans and $S$. aureus wild-type or an $\alpha$ toxin-deficient mutant $(\Delta h l a)$ and biomarkers of organ damage kinetically assessed in the serum. Polymicrobial infection with wild-type $S$. aureus led to significant increases in 3 common liver enzymes (alkaline phosphatase, ALP (Figure 4-2A); alanine transaminase, ALT (Figure 4-2B); aspartate aminotransferase, AST (Figure 4-2C)) and an increase in nitrogen blood levels (blood urea nitrogen, BUN (Figure 4-2D)) as compared to coinfection with the $\Delta$ hla strain. Given that $C$. albicans augments $\alpha$-toxin production in vivo, it is likely that increases in this potent virulence determinant leads to increased damage to the liver and kidneys contributing to mortality.

\section{$\alpha$-Toxin Must Be Functional for Lethal Synergism}

$\alpha$-toxin is secreted as monomeric units but adopts a heptameric form in the cell membrane which ultimately leads to pore formation $(141,149,150)$. Key studies have demonstrated that the $\mathrm{H} 35$ residue in the N-terminus is essential for stabilization of the heptamer and amino acid changes at this residue abolishes lytic activity $(151,152)$. Although mutated $\alpha$-toxin cannot form pores, it is still able to bind to ADAM10 in the membrane and could potentially activate a number of intracellular signaling cascades to drive pathogenicity. In order to determine whether toxigenic activity is required for lethality during IAI, we constructed a plasmid containing the entire hla ORF (pSK-hla) and used site-directed mutagenesis to introduce a single nucleotide change $(182 \mathrm{~A}<\mathrm{T})$ leading to a nonsynonymous substitution in amino acid sequence $(\mathrm{H} 35 \mathrm{~L})$. This plasmid was named pSK-H35L. Plasmids pSK-hla and pSK-H35L were transformed into an $\alpha-$ toxin null mutant, $\Delta$ hla. The hemolytic activity of the two complemented strains, $\Delta h l a-$ phla and $\Delta h l a-$ phla-H35L, was assessed by plating filter-sterilized supernatants from overnight cultures in wells on blood agar. As expected, the supernatant from $\Delta$ hla-phla formed a zone of hemolysis, while the H35L mutant strain did not, despite similar $\alpha$ toxin production (Figure 4-3A, B). Similar to prior reports, production of both $\alpha$-toxin isoforms was increased when co-cultured with $C$. albicans (Figure 4-3B). In vitro growth between these two complemented strains was not altered during in vitro mono- or polymicrobial conditions (Figure 4-3C). 

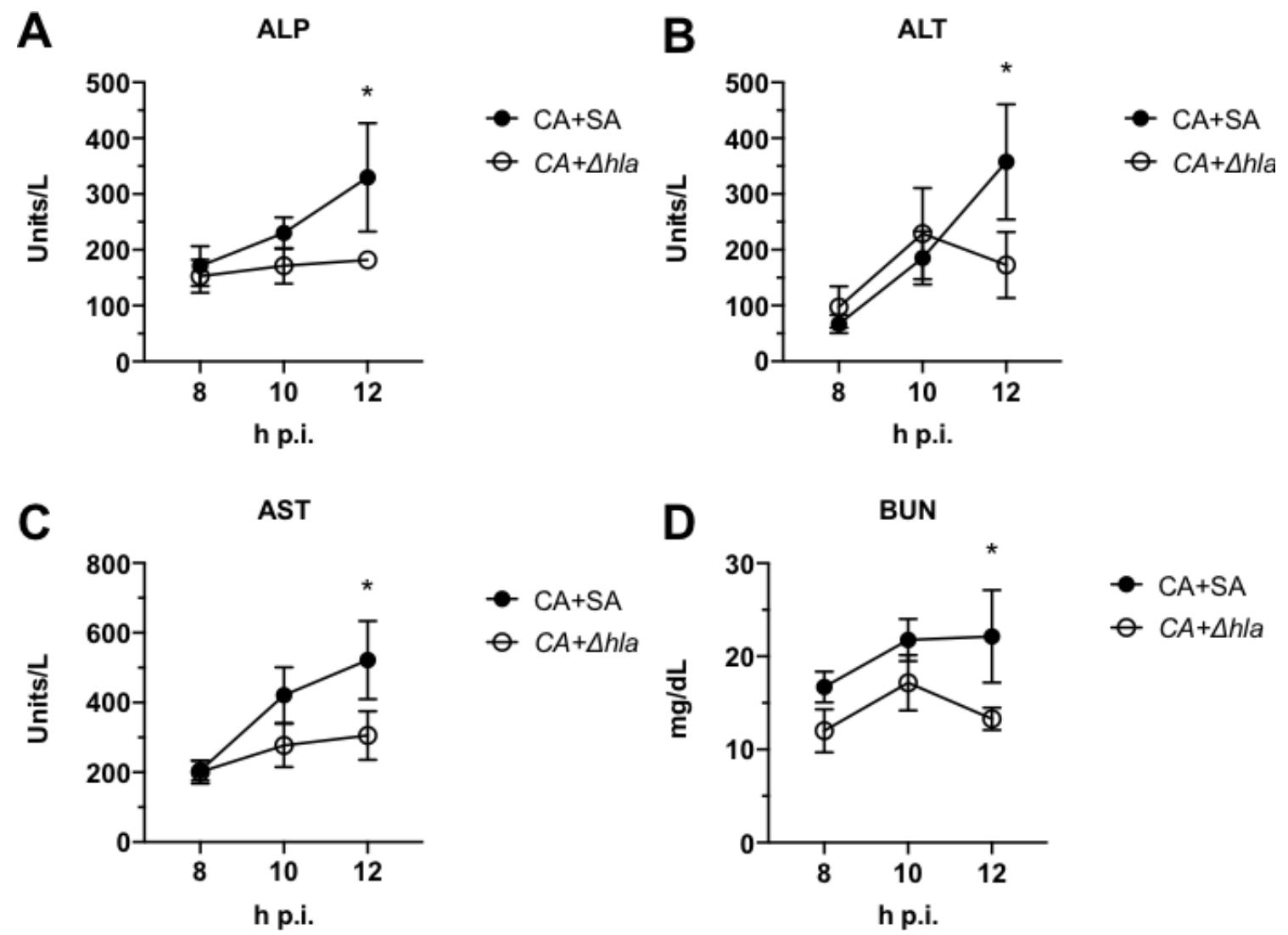

Figure 4-2. Kinetic changes in organ damage biomarkers.

Mice ( $n=8$ per group) were infected with $C$. albicans (CA) + wild-type $S$. aureus (SA) or an hla deletion mutant $(\Delta h l a)$ and sacrificed at 8,10 , or $12 \mathrm{~h}$ p.i. Levels of serum (A) alkaline phosphatate (ALP), (B) alanine transaminase (ALT), and (C) aspartate aminotransferase (AST) were measured as indicators of liver damage and (D) blood urea nitrogen (BUN) levels were assessed. Significance was determined using an unpaired multiple t-test. ${ }^{*}, P<0.05$. 
Figure 4-3. $\alpha$-toxin activity is required for lethal synergism during polymicrobial IAI.

(A) Filter-sterilized supernatants from S. aureus $\Delta$ hla-phla and $\Delta h l a-p h l a-H 35 \mathrm{~L}$ cultures were added to wells in blood agar plates and incubated at $37^{\circ} \mathrm{C}$ for $24 \mathrm{~h}$. Plates were scanned and images are representative of at least three independent repeats. (B) The amount of $\alpha$-toxin in S. aureus $\Delta$ hla-phla (solid) and $\Delta$ hla-phla-H35L (hashed) $\pm C$. albicans (mono, yellow; poly, green) culture supernatants was measured via ELISA. Experiment was repeated in triplicate and expressed as mean \pm SEM. Significance was determined by two-tailed Student's t-test. *, $P<0.05$; **, $P<0.01$. (C) CFU levels $(C$. albicans, blue; $S$. aureus strains, yellow) of monomicrobial and polymicrobial cultures at $16 \mathrm{~h}$ growth were assessed by microbiological plating on selective media. Counts were assessed for significance using a Mann-Whitney $\mathrm{U}$ test. (D) Mice ( $\mathrm{n}=8$ per group) were infected with $C$. albicans (CA) and wild-type $S$. aureus (SA, black line), an hla-deficient mutant ( $\Delta h l a$, red line), an hla-complemented strain ( $\Delta h l a-$ phla, blue line) or the oligomerization-deficient complemented strain ( $\Delta$ hla-phla-H35L, purple line) using standard inocula. Survival was followed for up to $5 \mathrm{~d}$ p.i. Data are of two independent repeats of 4 mice per group and combined. Significance was assessed by Gehan-BreslowWilcoxon test. $* * * *, P<0.001$. (E) Microbial burdens were enumerated in the peritoneal lavage fluid and homogenized spleen and kidneys by microbial plating on selective media. Data is expressed as mean \pm SEM. Significance was determined using an unpaired two-tailed Student's t-test. (F) Levels of $\alpha$-toxin found in the peritoneal lavage and homogenized spleen and kidneys were measured by ELISA. Data is cumulative of two independent repeats and expressed as mean \pm SEM. Significance was determined by Mann-Whitney test. Ns, non-significant. 


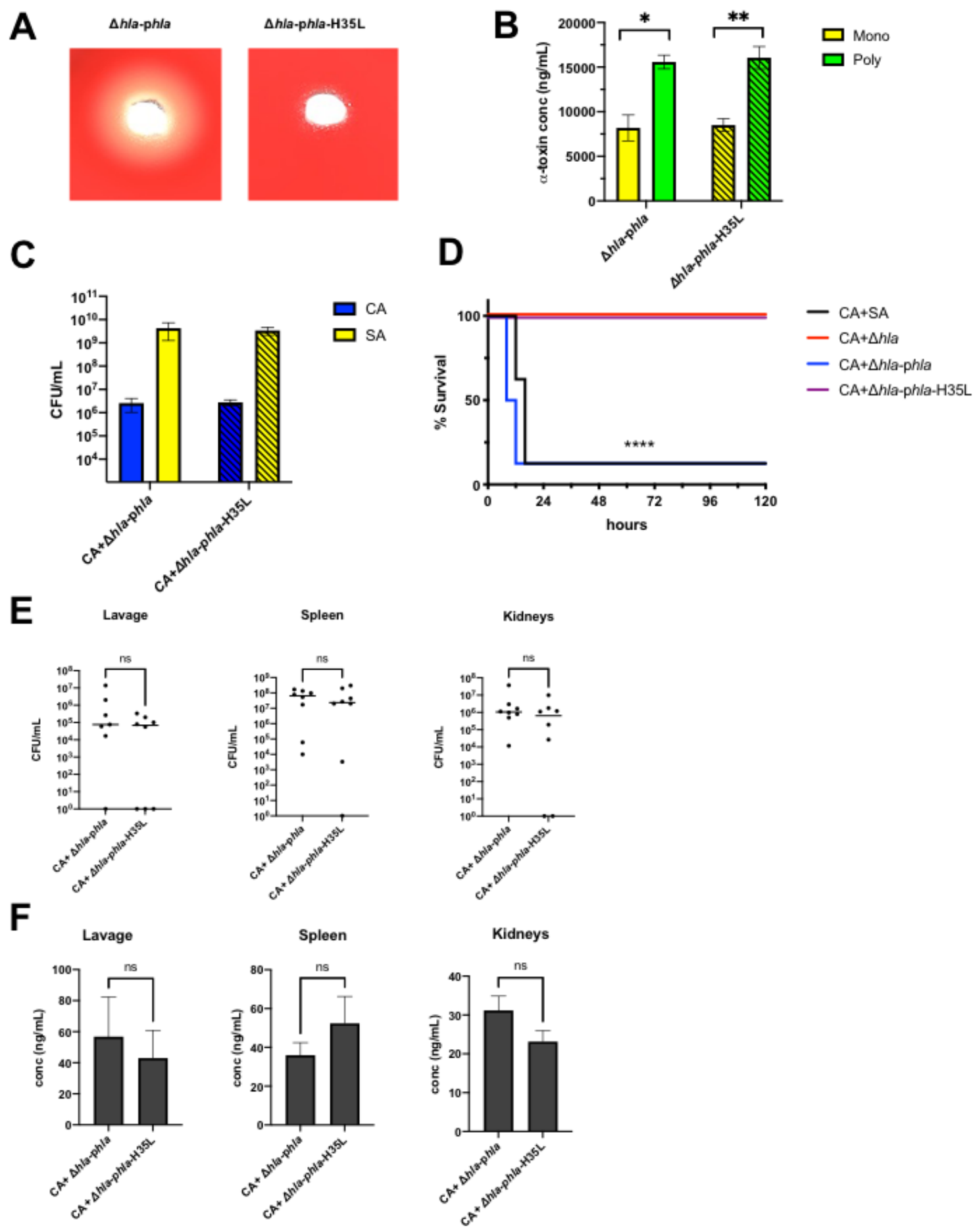


We next sought to determine whether the H35L mutant strain was able to induce synergistic lethality during polymicrobial IAI with $C$. albicans. Mice were infected i.p. with wild-type $C$. albicans and S. aureus JE2, $\Delta$ hla, $\Delta$ hla-phla, or $\Delta$ hla-phla-H35L and survival was followed for up to $5 \mathrm{~d}$ p.i. Mice infected with wild-type (JE2) $S$. aureus and the wild-type hla-complemented strain succumbed to infection within $24 \mathrm{~h}$ p.i., whereas infection with the $\alpha$-toxin-null and H35L mutant was nonlethal (Figure 4-3D). Notably, there were no significant differences in the amount of $\alpha$-toxin present or bacterial burden in the kidneys, spleen, and peritoneal lavage fluid of mice infected with the two complemented strains (Figure 4-3E, F). These data indicate that the oligomerization and cytolytic activity of $\alpha$-toxin is required to drive lethality during polymicrobial IAI.

\section{Several C. albicans Transcription Factors Are Important for Enhancing S. aureus $\alpha$ - Toxin Production}

While staphylococcal $\alpha$-toxin is necessary for lethality, the mechanism(s) by which $C$. albicans augments its production both in vitro and in vivo remains undefined. Despite the strong impact $C$. albicans-mediated alkalinization has on $S$. aureus agr activity in vitro, an alkalinization-deficient mutant was still able to induce lethal synergism during polymicrobial IAI (122) (Figure 4-1). In order to identify other potential candidal factors involved in enhancing $\alpha$-toxin production, an unbiased screen was undertaken. Mutants from a transcription factor deletion library were co-cultured with an $S$. aureus agr reporter strain $(S$. aureus(pDB22)) $(65,122,125,143)$. Foldchange fluorescence of polymicrobial cultures was normalized to $S$. aureus(pDB22) monoculture (Figure 4-4A). The TF WT control strain (indicated by pink dots) displayed a consistent 2-2.5-fold increase in signal. We found 9 mutants in the initial screen that failed to enhance $a g r$ activity ( $\geq 2$ standard deviations of the mean, gray dotted lines) to the same extent as TF WT. Follow-up assays confirmed that these mutants exhibited a defect in augmenting agr activity (Figure 4-4B). These mutants are listed in Table 4-3. The defect in $a g r$ activation of these mutants also extended to an inability to enhance $\alpha$ toxin production (Figure 4-4C). CFU counts from polymicrobial cultures revealed that $\Delta / \Delta$ bas $1, \Delta / \Delta l e u 3$, and $\Delta / \Delta m s n 4$ had significant growth defects that likely explained their inability to enhance agr activity; therefore, these mutants were excluded from further analysis (Figure 4-4D). As the agr quorum sensing system is responsive to $\mathrm{pH}$ and Candida-mediated alkalinization is a strong driver of $a g r$ activity in vitro, we determined whether these mutants had alkalinization defects that could explain their inability to augment agr activity $(115,122,123) . \mathrm{pH}$ measurements demonstrated that $\Delta / \Delta s f l 1$, $\Delta / \Delta \operatorname{grf} 10$, and $\Delta / \Delta i s w 2$ exhibited significant alkalinization defects, which could be attributing to the defective phenotype observed (Figure 4-4E).

We next evaluated the contribution of these 6 transcription factors in driving synergistic lethality during polymicrobial IAI. Mice were infected with SA and either the TF WT or deletion mutants and followed for survival (Figure 4-5A). Although the majority of the mutants elicited normal lethality, $\Delta / \Delta s f l$ (red line) and $\Delta / \Delta z c f 13$ (green line) had attenuated virulence (50 and $0 \%$ mortality, respectively). We evaluated the 

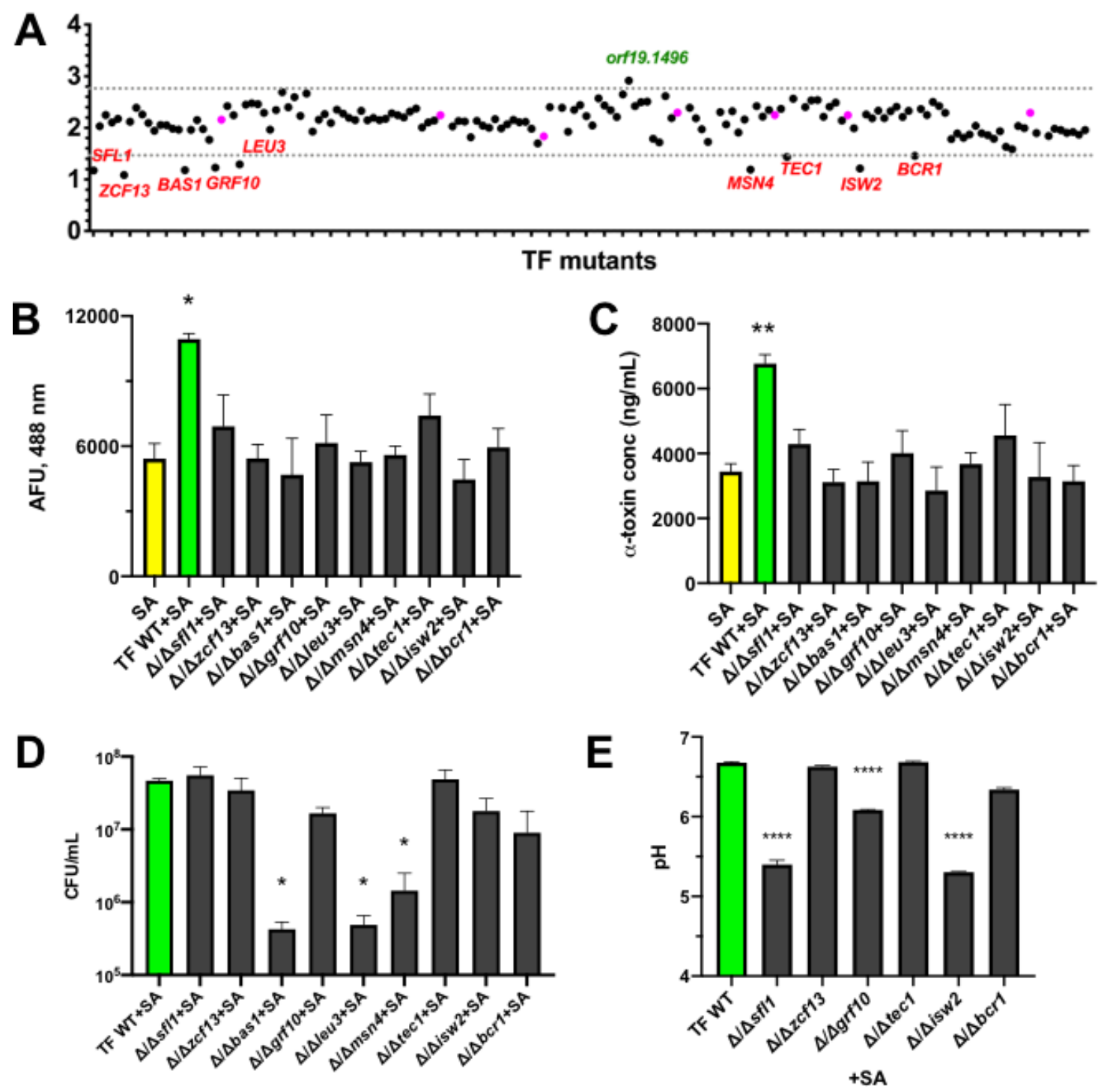

Figure 4-4. Screen of Candida transcription factor mutants reveals novel regulators of $S$. aureus agr induction.

(A) TF WT (pink dots) or mutants (black dots) were grown with $S$. aureus(pDB22) in polymicrobial cultures, fluorescence $(488 / 515 \mathrm{~nm})$ measured at $16 \mathrm{~h}$, and plotted as foldfluorescence over $S$. aureus monomicrobial control. Mutants exhibiting altered fluorescence changes are labeled in red (reduced) or green (enhanced). Dashed lines represent 2 standard deviations from the population mean. Mutants identified in (A) were confirmed for defect in (B) agr enhancement and (C) $\boldsymbol{\alpha}$-toxin production. (D) Growth of Candida strains in polymicrobial culture was measured by plating of serial dilutions of culture onto selective media. (E) The $\mathrm{pH}$ of polymicrobial cultures were measured at $16 \mathrm{~h}$ p.i. All experiments were repeated in biological triplicate and represented as the mean \pm SEM. Significance was assessed using one-way ANOVA and Dunnett's posttest. *, P < $0.05 ; * *, \mathrm{P}<0.01 ; * * * *, \mathrm{P}<0.0001$. 
Table 4-3. C. albicans transcription factor mutants identified in screen to have defects in augmenting $S$. aureus agr activity.

\begin{tabular}{|c|c|c|c|c|}
\hline $\begin{array}{c}\text { TF } \\
\#\end{array}$ & ORF & $\begin{array}{l}\text { Gene } \\
\text { Name }\end{array}$ & Description/Function & References \\
\hline 001 & 19.454 & SFL1 & $\begin{array}{l}\text { Suppressor of flocculation; negative } \\
\text { regulator of morphogenesis, } \\
\text { flocculation; deletion and } \\
\text { overexpression results in attenuated } \\
\text { virulence. }\end{array}$ & $(153,154)$ \\
\hline 006 & 19.2646 & ZCF13 & $\begin{array}{l}\text { Uncharacterized; predicted zinc-cluster } \\
\text { transcription factor; mutant has } \\
\text { decreased colonization of mouse } \\
\text { kidneys. }\end{array}$ & $(155,156)$ \\
\hline 016 & 19.3809 & $B A S 1$ & $\begin{array}{l}\text { Regulator of purine biosynthetic genes; } \\
\text { mutants are adenine auxotrophs. }\end{array}$ & $(157,158)$ \\
\hline 021 & 19.4000 & GRF10 & $\begin{array}{l}\text { Involved in regulation of filamentation, } \\
\text { biofilm formation, adenylate } \\
\text { biosynthesis; null mutants exhibit } \\
\text { leaky adenine auxotrophy and } \\
\text { decreased filamentation; promoter } \\
\text { bound by } B C R 1, T E C l, E F G 1 \text {, } \\
\text { NDT80, BRG1. }\end{array}$ & $(157,159)$ \\
\hline 025 & 19.4225 & LEU3 & $\begin{array}{l}\text { Predicted regulator of branched-chain } \\
\text { amino acid biosynthesis genes. }\end{array}$ & $(160,161)$ \\
\hline 109 & 19.4752 & MSN4 & $\begin{array}{l}\text { Uncharacterized zinc finger } \\
\text { transcription factor; not a significant } \\
\text { stress response regulator. }\end{array}$ & $(162)$ \\
\hline 115 & 19.5908 & TECI & $\begin{array}{l}\text { Hyphal gene regulator; regulates } \\
B C R 1 ; \text { upregulated in alkaline } \\
\text { conditions; regulates pheromone } \\
\text { response of white cell phenotype; } \\
\text { regulated by Efg } 1 \mathrm{p} \text { and Cph2p. }\end{array}$ & $(163-165)$ \\
\hline 127 & 19.7401 & ISW2 & $\begin{array}{l}\text { ATPase involved in chromatin } \\
\text { remodeling; required for } \\
\text { chlamydospore formation; mutants are } \\
\text { highly sensitive to fluconazole. }\end{array}$ & (166) \\
\hline 137 & 19.723 & $B C R 1$ & $\begin{array}{l}\text { Required for formation of biofilms; } \\
\text { involved in hyphae formation; } \\
\text { regulated by } T E C 1 \text {; controls expression } \\
\text { of Als3p. }\end{array}$ & (158) \\
\hline
\end{tabular}

Gene information acquired from the Candida Genome database (CGD): http://www.candidagenome.org 
A

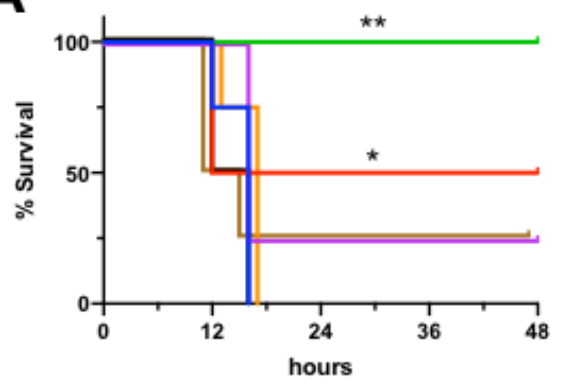

C

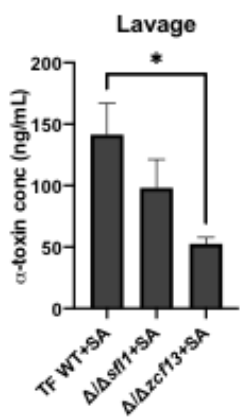

- TF WT+SA

- $\Delta / \Delta$ sfl1 + SA

- $\Delta \Delta z c f 13+S A$

- $\Delta \Delta g r f 10+S A$

- $\Delta \Delta \Delta i s w 2+S A$

- $\triangle \triangle \Delta b c r 1+S A$

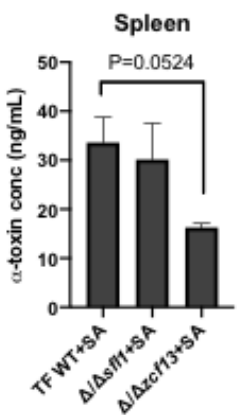

- $\Delta \Delta \operatorname{\Delta tec} 1+\mathrm{SA}$

B
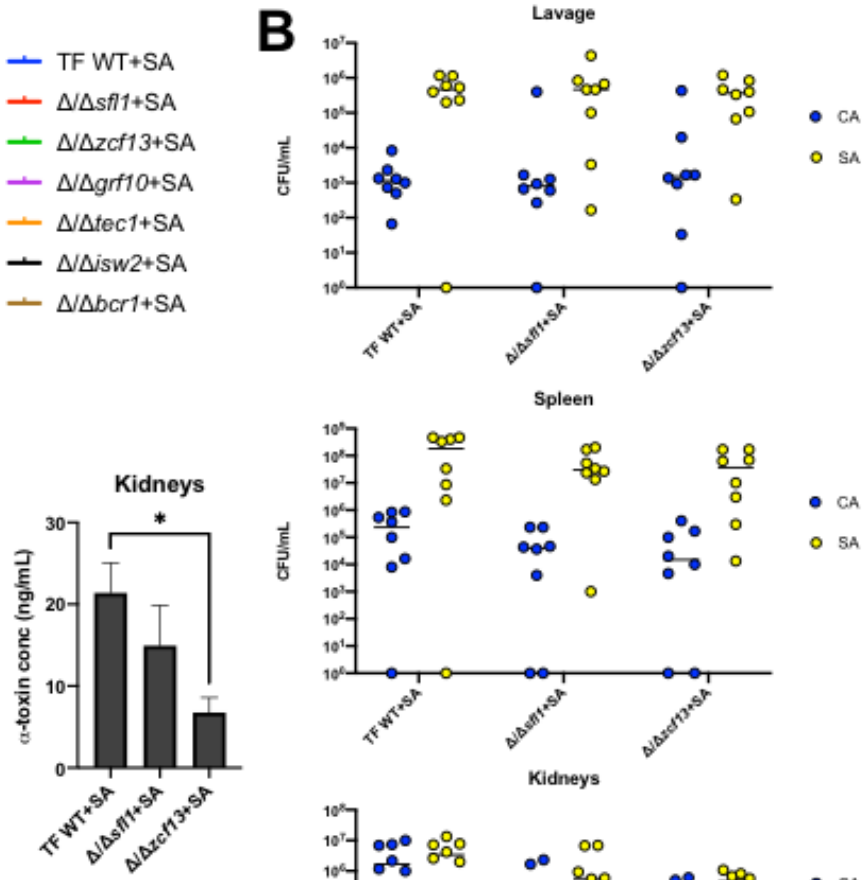

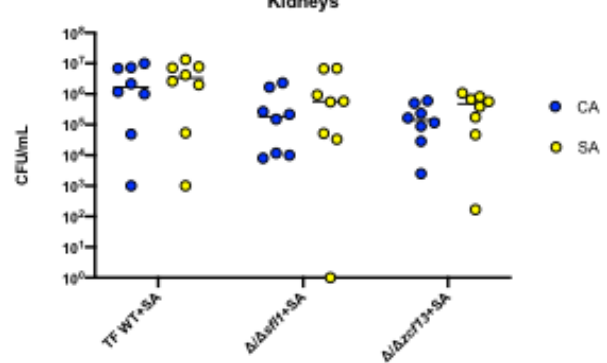

Figure 4-5. $\quad \Delta / \Delta s f l 1$ and $\Delta / \Delta z c f 13$ mutants fail to cause synergistic lethality during polymicrobial infection.

(A) Mice ( $\mathrm{n}=8$ per group) were infected with SA and either TF WT or select previously identified mutants $(\Delta / \Delta s f l 1, \Delta / \Delta z c f 13, \Delta / \Delta g r f 10, \Delta / \Delta t e c 1, \Delta / \Delta i s w 2, \Delta / \Delta b c r l)$ and followed for survival. Experiments were repeated twice and data combined. Significance was assessed using a Wilcoxon log rank test. (B) Microbial burdens in the peritoneal lavage fluid and homogenized spleen and kidneys were enumerated by microbiological plating at $8 \mathrm{~h}$ p.i. by selective microbiological plating. Line represents the median.

Significance was determined using Mann-Whitney test. (C) Levels of $\alpha$-toxin were also assessed in the peritoneal lavage fluid and homogenized spleen and kidneys by ELISA. Data is represented as mean \pm SEM. Significance was determined using a one-way ANOVA test. $*, P<0.05$; $* *, P<0.01$. 
microbial burden in the kidneys, spleen, and peritoneal lavage fluid of mice infected with TF WT, $\Delta / \Delta s f l l$, or $\Delta / \Delta z c f 13$ with SA $8 \mathrm{~h}$ p.i. and found no significant differences in colonization (Figure 4-5B). Despite similar growth, the $\Delta / \Delta z c f 13$ mutant was unable to enhance $S$. aureus $\alpha$-toxin production in the kidneys, spleen, and peritoneal cavity to the same level as the TF WT strain (Figure 4-5C). This data suggests that the transcription factor ZCF13 is involved in modulating $S$. aureus virulence during polymicrobial IAI.

\section{ZCF 13 Is Necessary for Candida-Induced agr Activation}

In order to confirm results obtained with the library deletion strain, independent mutant (SC $\Delta / \Delta z c f 13$ ) and revertant (SC $\Delta / \Delta z c f 13-\mathrm{Rev}$ ) strains were constructed in the SC5314 background using the SAT1-flipper method (146). We confirmed that this independent SC $\Delta / \Delta z c f 13$ mutant is deficient in enhancing agr activity and $\alpha$-toxin during co-culture, with no observable differences during mono- or polymicrobial growth (Figure 4-6A, B, C). Additionally, the ZCF13 revertant performed similarly to SC5314, although we observed a modest, yet significant decrease in CFUs (Figure 4-6A, B, C). We then co-infected mice with $S$. aureus and SC5314, SC $\Delta / \Delta z c f 13$, or SC $\Delta / \Delta z c f 13-\mathrm{Rev}$ and followed survival for up to $6 \mathrm{~d}$ p.i. While SC $\Delta / \Delta z c f 13$ did induce some mortality, it was significantly delayed and attenuated compared to wild-type and revertant polymicrobial infection (Figure 4-6D). Importantly, we did not see any colonization defects between the three strains in the kidneys, spleen, or peritoneal lavage fluid (Figure 4-6E). However, SC $\Delta / \Delta z c f 13$ was unable to enhance $\alpha$-toxin production to the same levels seen with SC5314 or SC $\Delta / \Delta z c f 13$-Rev (Figure 4-6F). This data indicates a crucial role for ZCF13 in the synergistic lethality observed during polymicrobial IAI with C. albicans and S. aureus.

\section{Spatiotemporal Induction of the agr Quorum Sensing System In Vivo}

Finally, we aimed to visualize the spread and activation of the agr quorum sensing system during mono- and polymicrobial IAI using a luciferase reporter driven by the staphylococcal P3 promoter. Similar to the P3-GFP reporter, the luciferase reporter responded comparably during mono and coculture (Figure 4-7A). Mice were infected with $S$. aureus(pOLux) with or without wild-type and $\Delta / \Delta z c f 13 C$. albicans strains. Luminescence was captured and images taken at $4 \mathrm{~h}$ intervals (Figure 4-7B). The luminescence intensity, expressed as the average of counts in regions of interest (ROI), at each time point is shown in Figure 4-7C. Although not statistically significant, wild-type polymicrobial infections trended higher luminescence at each time point, and demonstrated more dispersed agr signal in the abdomen, as compared to $S$. aureus monoor poly-infection with $\Delta / \Delta z c f 13$ mutants. All mice exhibited pockets of intense luminescence signal, which may represent high agr activity within the liver, spleen, and/or kidneys, but this was much more pronounced in mice co-infected with wild-type C. albicans. This qualitative approach further supports the hypothesis that $C$. albicans enhances $a g r$ activity during infection in a ZCF13-dependent manner. 
Figure 4-6. Deletion of ZCF13 in SC5314 abrogates $S$. aureus toxin production and synergistic lethality.

S. aureus(pDB22) was grown alone or with $C$. albicans SC5314, SC $\Delta / \Delta z c f 13$, or SC $\Delta / \Delta z c f 13-\mathrm{Rev}$ in TSB-g at $37^{\circ} \mathrm{C}$ with shaking. At $16 \mathrm{~h}$ p.i., aliquots were removed to (A) measure fluorescence, $(\mathbf{B})$ enumerate $\mathrm{CFUs}$, or $(\mathbf{C})$ measure $\alpha$-toxin levels. Experiments were repeated in triplicate and data are expressed as mean \pm SEM. (D) Mice $(n=8$ per group) were infected with $7 \times 10^{6}$ CFU SC5314, SC $\Delta / \Delta z c f 13$, or SC $\Delta / \Delta z c f 13-\mathrm{Rev}+$ $8 \times 10^{7} \mathrm{CFU}$ S. aureus i.p. and monitored for survival for up to $5 \mathrm{~d}$ p.i. Experiments were performed in duplicate and combined. Significance was assessed using a Wilcoxon log rank test. (E) Microbial burdens at $8 \mathrm{~h}$ p.i. in lavage fluid and homogenized spleen and kidneys of mice infected with SA and SC5314, SC $\Delta / \Delta z c f 13$, or SC $\Delta / \Delta z c f 13$-Rev were enumerated by microbiological plating on selective media. Data are cumulative of two independent repeats and expressed as the median. (F) Levels of $\alpha$-toxin at $8 \mathrm{~h}$ p.i. in peritoneal lavage fluid and homogenized spleen and kidneys from mice infected with SA and SC5314, SC $\Delta / \Delta z c f 13$, or SC $\Delta / \Delta z c f 13$-Rev were measured via ELISA. Data is cumulative of two independent repeats and represented as mean \pm SEM. Significance was determined using a one-way ANOVA and Dunnett's posttest ( $\alpha$-toxin), Mann-Whitney test (CFU), and Wilcoxon log rank test (survival). ${ }^{*}, P<0.05$; **,$P<0.01$; ***, $P<$ 0.001 . 

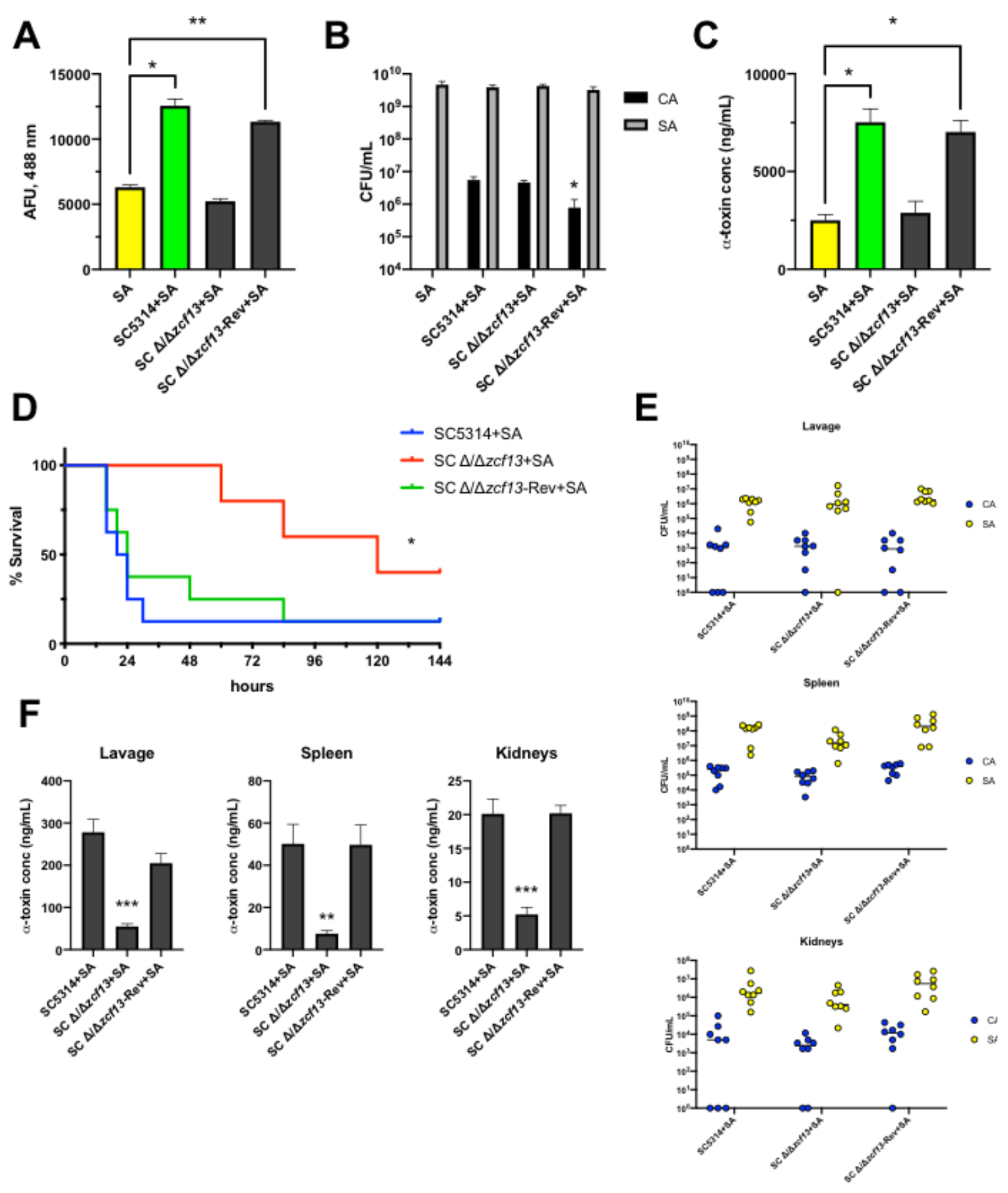
Figure 4-7. Spatiotemporal agr activation during mono- and polymicrobial infection with WT and $\Delta / \Delta z c f 13$.

(A) Luminescence was measured at $16 \mathrm{~h}$ p.i. in mono- and polymicrobial cultures of $S$. aureus(pOLux) with or without $C$. albicans SC5314, SC $\Delta / \Delta z c f 13$, TF WT, or TF $\Delta / \Delta z c f 13$ grown in TSB-g at $37^{\circ} \mathrm{C}$ with shaking. Data is cumulative of three independent experiments and expressed as mean \pm SEM. Significance was determined using a oneway ANOVA and Dunnett's posttest. *, $P<0.05$; **, $P<0.01$. (B) Mice were infected with $S$. aureus(pOLux) $\pm C$. albicans SC5314, SC $\Delta / \Delta z c f 13$, TF WT, or TF $\Delta / \Delta z c f 13$.Images were taken every $4 \mathrm{~h}$ p.i. using Xenogen IVIS Spectrum. Images are uniformly scaled. (C) Luminescence values were quantified within regions of interest and plotted as mean \pm SEM. Significance was assessed using a one-way ANOVA and Dunnett's posttest. Ns, non-significant. 


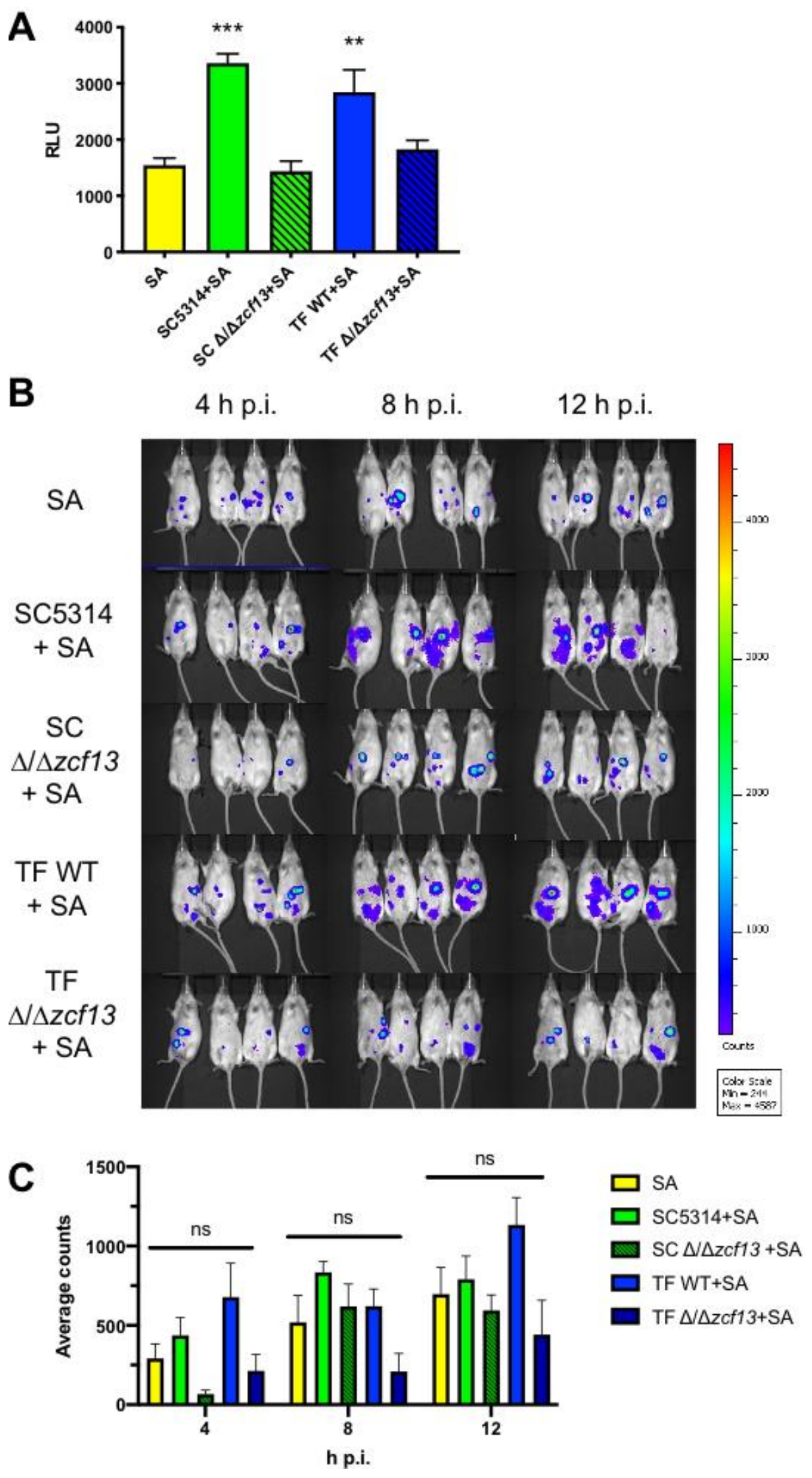




\section{Conclusions}

Although microbes naturally exist in mixed populations, the interactions occurring between such organisms are understudied and poorly understood partially due to their often highly complex and multi-factorial nature. Our lab has focused on delineating the mechanisms of interaction between $C$. albicans and S. aureus during polymicrobial growth and infection. Early studies by Carlson revealed a lethal synergism between these pathogens during polymicrobial IAI $(59,71)$. We previously identified the cytolytic exotoxin, $\alpha$-toxin, as the staphylococcal effector of virulence, as co-infection of an $\alpha$-toxin-null mutant $(\Delta h l a)$ with $C$. albicans was nonlethal and protection could be achieved by targeting it with a monoclonal antibody (65). $\alpha$-toxin is a multifactorial toxin that, in addition to its cytolytic activity, is able to stimulate a pro-inflammatory response and disrupt platelet function. During S. aureus-induced sepsis, Surewaard et al. found significant platelet aggregations in the livers of infected mice that were absent when an H35L mutant $\alpha$-toxin was used or when mice were passively immunized with an anti- $\alpha$ toxin antibody, MEDI4893* (142). Circulating platelets were decreased, indicating the induction of thrombocytopenia due to $\alpha$-toxin. Additionally, the liver-associated enzyme ALT was increased during infection and was correlated with focal necrotic lesions in this organ (142). A related study by Powers et al. demonstrated that $\alpha$-toxin alters platelet activation as well as promotes platelet-neutrophil aggregates, which contributes to damage to lung and liver tissue (58). Consistent with these findings, we also observed significantly increased levels of biomarkers of kidney and liver damage during coinfection and these correlated with increased $\alpha$-toxin during co-infection (Figure 4-2). While this relationship is not definitively causative, it is likely that organ damage due to $\alpha$-toxin is a major contributor to lethality during murine IAI. It is possible that $\alpha$-toxin is disrupting the host coagulation cascade, leading to decreased circulating platelets and the formation of platelet aggregates within the organs, consistent with multi-organ failure seen in severe sepsis (167). However, future studies must be conducted to answer this question.

Our lab has demonstrated that $C$. albicans enhances $\alpha$-toxin production in vitro and in vivo $(65,122)$. However, the mechanism of this inter-kingdom interaction was unclear. In order to identify and characterize the candidal factors involved in S. aureus agr activation, we screened a collection of $C$. albicans transcription factor deletion mutants. Several diverse transcriptional regulators displayed an inability to augment $S$. aureus agr activity and $\alpha$-toxin production in vitro (Table 4-3). Three mutants $(\Delta / \Delta b a s l$, $\Delta / \Delta l e u 3$, and $\Delta / \Delta m s n 4$ ) had significant growth defects, which potentially explains the lack of enhancement of agr activity seen in polymicrobial cultures. In fact, $\Delta / \Delta b a s l$ is a known adenine auxotroph, as it mainly functions to regulate purine biosynthesis genes, suggesting that adenine is limited in this media. GRF10 also plays a role in purine biosynthesis and mutants have been shown to have diminished growth in media lacking adenine, but this phenotype is not as severe as with BAS1 deletion (157).

An interesting finding from the transcription factor mutant screen is the identification of three mutants that have defects in alkalinization during growth with $S$. aureus. These transcription factors, SFL1, GRF10, and ISW2, have not previously been 
shown to play a role in $\mathrm{pH}$ modulation (127). However, ISW2 has been proposed to interact with alkaline $\mathrm{pH}$-response regulators during chlamydospore formation (166). Alkalinization and morphogenesis are interconnected processes, perhaps most relevant in macrophage phagosomes. $C$. albicans has evolved a way to counteract the acidic phagolysosome by releasing ammonia produced primarily through the breakdown of amino acids $(124,127)$. This neutralization permits transition from yeast to hyphae, allowing for escape from the macrophage. While alkalinization plays a clear role in driving staphylococcal $\alpha$-toxin induction in vitro, its role in vivo has not been validated, partially due to the buffered and slightly alkaline environment of the peritoneal cavity (68). Thus, it is difficult to determine whether partial loss of synergism in the $\Delta / \Delta s f l$ strain is due to its alkalinization defect or another uncharacterized phenotype.

Previous studies from our lab determined that morphology/morphogenesis are not drivers of enhanced lethality during polymicrobial IAI with C. albicans and S. aureus, as hypha-defective and constitutively filamentous strains induce the same mortality as wildtype $C$. albicans $(66,67)$. C. albicans does not form hyphae during growth in TSB-g, the media used in our in vitro assays, supporting this finding. However, three known regulators of hypha formation, GRF10,TEC1, and BAS1, seemed to be involved in enhancing $S$. aureus quorum sensing in vitro, as mutants were unable to augment $a g r$ activity. Other canonical inducers of filamentation, such as EFG1, CPH1, and TUP1, did not display defects, which may indicate alternative functions of $G R F 10, T E C 1$, and $B A S 1$ under these specific culture conditions.

Only one transcription factor mutant (TF \#089, orf19.1496) displayed enhanced agr augmentation, over 3-fold compared to $S$. aureus alone (Figure 4-4A, green dot). This transcription factor is uncharacterized, but its expression may be repressed by the iron-responsive Cap2-HAP complex (168). Though we did not further evaluate the contribution of this transcriptional regulator in the augmentation of $S$. aureus agr activity in this study, we hypothesize that polymicrobial IAI infection with this strain may be hypervirulent, although this may be difficult to distinguish given the robust and rapid lethality observed during wild-type co-infection.

We identified one transcription factor mutant that did not induce synergistic lethality (TF \#006, orf19.2646, ZCF13). ZCF13 is a predicted zinc-cluster transcription factor that is similar but not truly orthologous to the HAPl (Heme Activator Protein) transcription factor in $S$. cerevisiae, which is involved in regulation of genes in response to heme and oxygen $(169,170)$. While the function of ZCF13 is unknown, null mutants have been used in various phenotypic profiling experiments. These data can help eliminate potential functions of this regulator, although there are contradictory reports. For example, the initial screen of the library mutants in 55 different conditions did not detect any phenotypic abnormalities with this specific mutant. It exhibited no growth or morphological defects on a range of medias (YEPD, synthetic defined $+\mathrm{L}$-arginine and L-leucine, minimal media) at a range of temperatures $\left(16,30,37,42{ }^{\circ} \mathrm{C}\right)$ and had no observed sensitivities to antifungal drugs (fluconazole, fluphenazine, 5-fluorocytosine) or stressors (copper, zinc, caffeine, sodium dodecyl sulfate, hydrogen peroxide, etc.) (143). Conversely, in another study, using a separately constructed $\Delta / \Delta z c f 13$ mutant, it was 
found to be susceptible to increased temperature $\left(42{ }^{\circ} \mathrm{C}\right)$, exhibited increased agar invasion, and demonstrated increased colony wrinkling (155). It is possible that the parental strain in which these separate mutants were constructed could contribute to the different phenotypes reported. However, neither $\Delta / \Delta z c f 13$ mutant showed altered growth or morphological differences in our hands (Figure 4-8).

Additionally, transcriptomic analyses under different conditions (hyphaeinducing, acidic and basic $\mathrm{pH}$, high and low oxidative stress, nitrosative stress, and cell wall damaging conditions) have not shown any significant changes in ZCF13 expression (171-173). The expression was also unaltered in tissue culture with primary human umbilical vein endothelial cells (HUVECs) or immortalized oral epithelial cells (OKF6/TERT-2) (174). However, ZCF13 expression was decreased 3-fold when RBF1 was knocked out (175). $R B F 1$ (RPG-box-binding factor 1) is a transcription factor that is associated with mitochondrial and peroxisomal metabolism, as well as being a negative regulator of morphogenesis (175). Thus, it is intriguing to speculate that a $\Delta / \Delta r b f 1$ mutant may also be hypovirulent during co-infection. As peroxisomal metabolism is integral to fatty acid $\beta$-oxidation and the glyoxylic shunt, it will be important to determine if these metabolic pathways are integral to $C$. albicans pathogenesis in the peritoneal space. However, based on these cumulative results, Zcf13p does not appear to be responsive to growth conditions or involved in the responses to diverse stress. Using a combination of transcriptomic sequencing under mono and polymicrobial conditions and tagging of Zcf13p with a C-terminal protein tag and chromatin immunoprecipitation sequencing (ChIP-seq) approaches may reveal regulatory function of this neglected transcription factor.

Collectively, data in this manuscript identify Zcf13p, an uncharacterized $C$. albicans transcription factor, as a key regulator of trans-kingdom virulence and further highlights the complexity of clinically relevant fungal-bacterial interactions in vivo. 


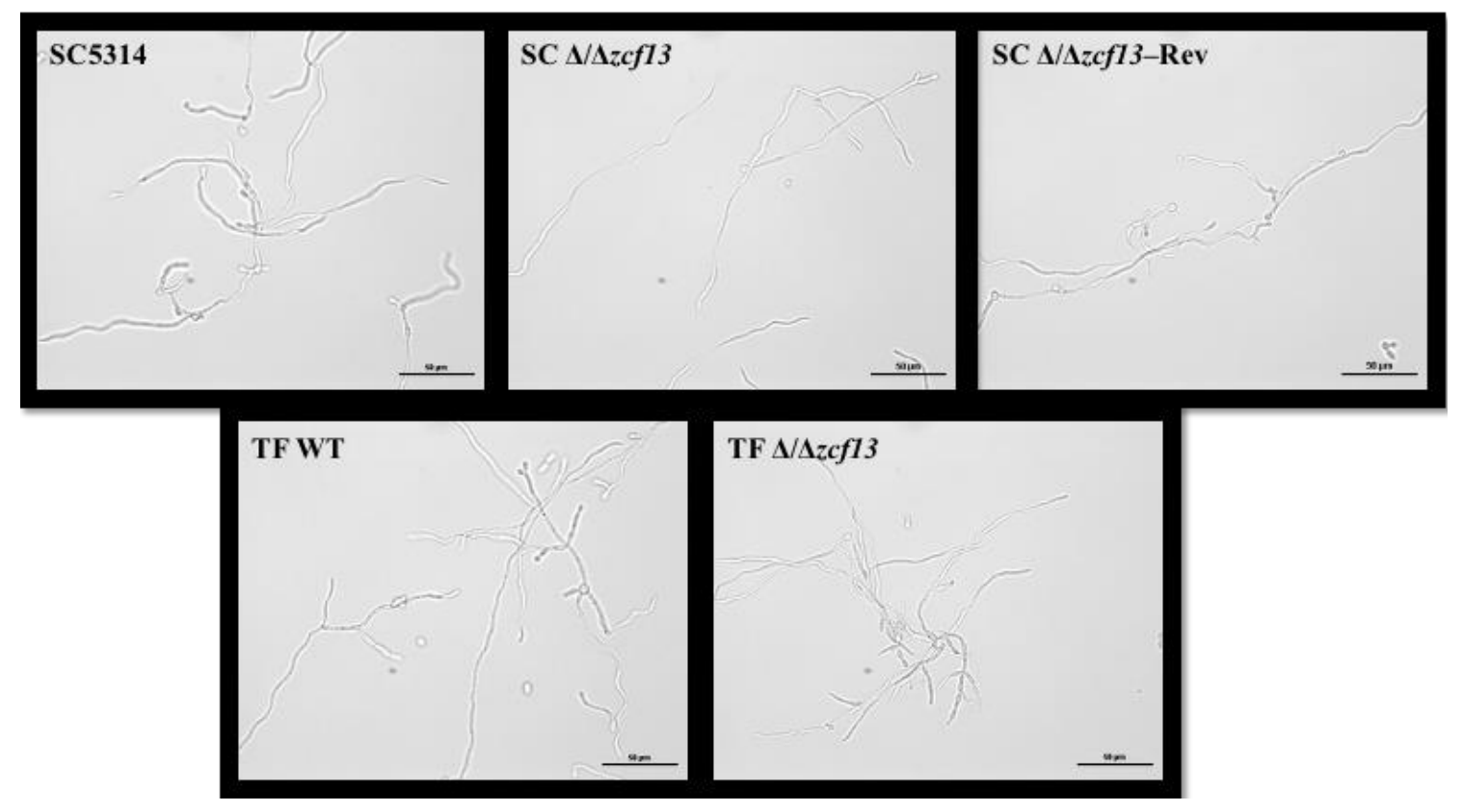

Figure 4-8. No differences in hyphal formation observed between wild-type and $\Delta / \Delta z c f 13$ strains.

Cells from overnight cultures of $C$. albicans SC5314, SC $\Delta / \Delta z c f 13$, TF WT, or TF $\Delta / \Delta z c f 13$ in YPD were washed and diluted into YNB and allowed to grow for $24 \mathrm{~h}$ at $37^{\circ} \mathrm{C}$ with shaking. Aliquots were removed and observed by microscopy. Images are representative of at least two independent repeats. Scale bar represents $50 \mu \mathrm{m}$. 


\section{CHAPTER 5. DISCUSSION AND FUTURE DIRECTIONS}

Candida albicans is a polymorphic, opportunistic fungal pathogen that is a major cause of fungal infections. Staphylococcus aureus is a pervasive bacterial pathogen capable of causing many different types of disease. While these organisms can cause significant morbidity and mortality on their own, they are also co-isolated from both biotic and abiotic surfaces on or within the human host. Although much research has focused on these microbes individually, their interaction in the context of disease is not as well understood. Early studies by Carlson described a mouse model of polymicrobial intra-abdominal infection (IAI) that revealed a synergism between $C$. albicans and $S$. aureus, which led to enhanced lethality compared to non-lethal monomicrobial infection (59). Despite years of study, this interaction has not been fully elucidated. Therefore, the purpose of this work was to answer several of the many remaining questions regarding the mechanism of synergistic lethality between C. albicans and S. aureus: What are the specific factors, both fungal and bacterial, that contribute to lethality? How does the host respond to polymicrobial infection, and is this response a significant cause of morbidity and mortality? What exactly is driving lethality: microbial growth and dissemination, organ damage, sepsis, hyperinflammatory responses? How can we prevent, detect, and effectively treat these types of infections?

In Chapter 2, we investigated the staphylococcal factors involved in polymicrobial IAI using genetic approaches, qualitative and quantitative functional assays, and a murine model of $C$. albicans-S. aureus infection. We found enhanced hemolysis during coculture that is both agr-and $\alpha$-toxin-dependent, as an hla-null mutant was unable to induce hemolysis in vitro and mortality during polymicrobial infection. Importantly, survival was greatly enhanced when mice were passively immunized with an anti- $\alpha$-toxin-antibody MEDI4893* prior to coinfection with $C$. albicans and $S$. aureus. These data indicate the main staphylococcal effector of synergistic lethality is $\alpha$-toxin and its production is significantly increased in the presence of $C$. albicans.

The strain used in this work (JE2) is a USA300 isolate which exhibits high $\alpha$ toxin expression (176). Previous studies using this model have used different $S$. aureus isolates that have vastly different toxin profiles. Carlson investigated toxic shock syndrome (TSS)-associated strains that were positive for toxic shock toxin (TST) and non-TSS-disease-associated strains (unknown toxin profile) during polymicrobial infection. She found that $S$. aureus strains that produced TST induced mortality starting at $35 \mathrm{~h}$ p.i. whereas the other non-TST strains led to $100 \%$ mortality by $15 \mathrm{~h}$ p.i. (71). This demonstrates that synergistic lethality is driven by $S$. aureus toxin but the type/activity of the toxin produced dictates the kinetics of infection. While these findings were undoubtedly important, the lack of isogenic strain pairs harboring toxin gene deletions was a major limitation to identifying the precise mechanism(s) driving synergism. In support of this, later work by Carlson suggested that $\delta$-toxin was the main driver of lethality during co-infection. Another group used strain NRS383 (USA200) in the mouse model of IAI, which is positive for TST and $\delta$-toxin but not $\alpha$-toxin.

Coinfection with this strain and C. albicans led to approximately $60 \%$ mortality by $2 \mathrm{~d}$ 
p.i. (62). While toxin levels during in vitro or in vivo growth with this strain have not been evaluated, it is likely that they would be elevated during growth with $C$. albicans. Despite differences in toxins, the in vivo infectious synergism is generally preserved with different $S$. aureus strains and $C$. albicans. However, $\alpha$-toxin is not the only toxin controlled by the $a g r$ system; others, including $\delta$-toxin and the PSMs, are also $a g r$ regulated. Interestingly, $S$. epidermidis (a close relative of $S$. aureus) also utilizes a similar $a g r$ system and its $\delta$-toxin demonstrates bacteriostatic effects on Group A streptococci, presumably conferring a selective advantage over other endogenous microbiota during skin colonization (177). Consequently, it is possible that additional toxins have growth inhibitory effects on $C$. albicans. As organisms that both largely asymptomatically colonize the human host, it would not be particularly advantageous to elicit such robust virulence and subsequent lethality. Thus, it is somewhat perplexing as to why $C$. albicans seemingly augments $S$. aureus agr signaling. It is conceivable that $\alpha-$ toxin could have an antagonistic effect on $C$. albicans, thereby allowing for a competitive advantage during co-culture. Preliminary studies to address this in our laboratory using purified $\alpha$-toxin neither inhibited $C$. albicans growth nor led to the uptake of propidium iodide to indicate fungal damage (unpublished data). Similarly, co-culture biofilm experiments using the Live/Dead staining system and fluorescence microscopy have revealed no apparent damage to hyphal cells. However, agr activity was not specifically addressed in these experiments, necessitating further interrogation of this hypothesis (47).

Peters et al. identified key changes in the immune response during polymicrobial versus monomicrobial IAI, with increases in proinflammatory cytokines and neutrophil recruitment. In order to determine whether the immune response contributes to morbidity and mortality, mice were treated with indomethacin prior to and during infection. Indomethacin is a nonsteroidal anti-inflammatory drug (NSAID) that nonselectively inhibits cyclooxygenase enzymes (COX-1, COX-2) that produce inflammatory prostaglandins from arachidonic acid, thereby reducing inflammation (178). Mice given the drug experienced significantly less morbidity as compared to vehicle-treated controls. Importantly, microbial burden in the kidneys and spleen was unaltered, suggesting that exacerbated immune responses may be driving lethality (62). Further experiments determined that the production of the prostaglandin $\mathrm{PGE}_{2}$ was increased during polymicrobial infection and indomethacin treatment abrogated this effect (62). $\mathrm{PGE}_{2}$ can be induced by $\alpha$-toxin, is highly immunomodulatory, and induces production of proinflammatory cytokines (e.g. IL-6, KC, G-CSF, MIP-1 $\alpha$ ), which may be to the detriment of the host when their production is uncontrolled. $(62,179)$. We measured PGE 2 levels during mono- and polymicrobial IAI and found they were significantly increased in the latter. Although we did not specifically test this, we hypothesize that treatment with indomethacin would also have a protective effect in our model. However, because the USA300 strain is able to produce significantly more toxin than a USA200 strain, indomethacin might not be as protective (62). Generally, COX inhibitors have not proven clinically useful against human sepsis, yet more fine-tuned targeting of individual prostaglandin receptors (EPs) has not been evaluated clinically (180). In support of this, treatment with EP1 and EP3 inhibitors have led to marked improvement of mice intraperitoneally challenged with $C$. albicans and $S$. aureus and may be a viable therapeutic strategy to limit mortality (87). 
While it is clear that inflammation and $\mathrm{PGE}_{2}$ signaling partially drive lethal outcome, $\alpha$-toxin has other effector functions beside membrane damage, lysis, and eicosanoid stimulation. It can activate signaling through its high-affinity host receptor, a disintegrin and metalloprotease 10 (ADAM10), which ultimately leads to disruption of tight junctions and tissue desquamation on endothelial and epithelial cells (181). $\alpha$-toxin has also recently been shown to activate platelet aggregation and dysregulate the hemostatic system, resulting in excessive clotting and liver injury due to exacerbated thrombosis (142). Interestingly, the MEDI4893* antibody that was protective against lethal challenge during polymicrobial IAI, also prevents such thrombotic events in the liver. Thus, it is conceivable that dysregulated hemostasis mediated by elevated levels of $\alpha$-toxin, followed by subsequent organ failure (defining features of sepsis and disseminated intravascular coagulation (DIC)) contribute to the synergistic mortality observed during polymicrobial IAI with $C$. albicans and $S$. aureus (182). Sepsis is a complex disease state in which the dysregulation of the host immune response and disruption of hemostasis (ranging from thrombocytopenia to DIC) leads to organ dysfunction, septic shock, and ultimately death if not diagnosed and adequately treated (183). Therefore, modulation of the clotting cascade may confer some level of protection during co-infection. In fact, a recent study demonstrated that the use of aspirin could prevent intravascular coagulation during $S$. aureus-induced sepsis in mice (184). Aspirin is another NSAID that irreversibly inhibits COX-1, mediates the activity of COX-2, and has antithrombotic properties (185). At a low dose in humans $(\sim 81 \mathrm{mg})$ it irreversibly inhibits platelet aggregation, thus making it a common treatment to prevent heart attacks caused by blood clots (185). Treatment with aspirin may have greater efficacy than indomethacin in preventing morbidity and mortality in our model, as it targets two potential host effectors contributing to lethality. Additionally, combining aspirin and MEDI4893* could synergistically enhance survival during polymicrobial IAI.

In Chapter 4 we correlated $\alpha$-toxin with organ damage biomarkers in the serum, specifically those indicating liver and kidney damage. Previous studies of staphylococcal sepsis found that $\alpha$-toxin is directly responsible for platelet aggregation and liver injury, assessed through measuring circulating platelet levels, serum ALT, and necrotic lesions within the liver and kidneys (142). Although the liver was not analyzed for microbial burden or $\alpha$-toxin production in our work, it is very likely that $\alpha$-toxin levels are elevated during polymicrobial IAI in this target organ as well. Based on this collective data, we hypothesize that the host succumbs to infection due to the multifactorial effects of $\alpha$ toxin: cellular and tissue damage, exaggerated platelet aggregation, and hyperactive immune responses.

In Chapter 3 we aimed to identify the candidal factor(s) involved in enhancing $S$. aureus toxin production. We first tested the hypothesis that a $C$. albicans effector directly interacts with the $a g r$ system via recognition by AgrC, but experimentally determined this was not a likely mechanism. Based on prior reports that the agr system is responsive to $\mathrm{pH}$, we hypothesized that $C$. albicans modulates the $\mathrm{pH}$ during co-culture to optimum levels. Indeed, we found that $C$. albicans alkalinizes the media and this correlated with higher agr activity. An alkalinization-deficient $C$. albicans mutant, $\operatorname{stp} 2 \Delta / \Delta$, was unable to raise the $\mathrm{pH}$ during in vitro growth with $S$. aureus and was also unable to enhance $\alpha$ - 
toxin levels. In support of this, different Candida species displayed disparate alkalinization potentials, with those able to raise the $\mathrm{pH}$ also inducing higher $\alpha$-toxin production (C. albicans, $C$. krusei, $C$. tropicalis). Species with defects in alkalinization were unable to augment toxin levels (C. dubliniensis, $C$. glabrata, $C$. parapsilosis). This data strikingly matches in vivo mortality data using the same Candida species in the murine polymicrobial IAI model (67). Although C. albicans-mediated alkalinization plays a role in synergism with $S$. aureus in vitro, this unfortunately did not translate in vivo, as the $\operatorname{stp} 2 \Delta / \Delta$ strain was lethal similar to wild-type co-infection (Figure 4-1). It's possible that other mechanisms of $\mathrm{pH}$ modulation exist, or that $\mathrm{pH}$ in combination with other factors, such as metabolic byproducts, are also involved. Biological $\mathrm{pH}$ is strictly maintained within different anatomical sites and it is entirely possible that $C$. albicans cannot cause large, global shifts. Perhaps this effect is only seen in the microenvironment when $C$. albicans and $S$. aureus are in close proximity, as commonly observed in polymicrobial biofilms. These two organisms have been shown to form biofilms together and that gene expression is altered during this growth modality (47). However, S. aureus $\alpha$-toxin production or $\mathrm{pH}$ changes have not been directly examined during biofilm growth. Experiments to determine if $\mathrm{pH}$ drives agr activity could be performed using the $\operatorname{stp} 2 \Delta / \Delta$ strain and wild-type $C$. albicans to answer this question in a potentially more relevant system.

Given the challenges faced with interrogating the impact of $\mathrm{pH}$ on driving lethality in vivo, in Chapter $\mathbf{4}$ we undertook an unbiased screening approach to identify other candidal factors that influence $S$. aureus agr activity and $\alpha$-toxin production. We identified several transcription factors that appeared to be necessary for this specific interaction: SFL1, ZCF 13, GRF10, TEC1, ISW2, and BCR1. Despite deficiencies seen in vitro, only one of these mutants failed to synergize with $S$. aureus in vivo: $\Delta / \Delta z c f 13$. This transcription factor is uncharacterized and its function unknown. Our lab is currently performing transcriptomic and metabolomic analyses to uncover the role that ZCF13 plays during the interaction between $C$. albicans and $S$. aureus. We also plan to utilize chromatin immunoprecipitation sequencing (ChIP-seq) to identify DNA-binding sites of $Z C F 13$, which may illuminate genes that are regulated by this transcription factor. It is possible that expression of ZCF 13 is altered in the presence of $S$. aureus or other bacteria and is involved in inter-microbial interactions. Other possible functions could be related to production of metabolic byproducts that impact $S$. aureus quorum sensing or protection from immune cells during infection. S. aureus virulence has been shown to be altered in response to pyruvate, an important molecule in cellular metabolism (186). Perhaps Zcf13p regulates genes that, under certain conditions, alter pyruvate production or secretion. Since ZCF 13 in $C$. albicans is orthologous in sequence to HAP1 in $S$. cerevisiae, it may be involved in iron-responsive pathways. Iron is an essential element but is tightly sequestered in the human body, leading to microbes evolving mechanisms for iron acquisition (187). It's possible that $C$. albicans and $S$. aureus compete for acquisition within the host, thereby leading to enhancement of virulence mechanisms. Future studies investigating the survival of $\Delta / \Delta z c f 13$ mutants within macrophages or metabolic profiling of mutants in the presence of $S$. aureus could answer these questions. Putative ZCF13 orthologs exist in the various Candida species utilized in Chapter 3 (CPAR2_501580 in C.parapsilosis; Cd36_53120 in C.dubliniensis; CAGL0B03421g in 
C. glabrata; CTRG_02695 in C. tropicalis) and potential functional differences may correlate with the ability to cause lethal synergism $(188,189)$.

In conclusion, this work identifies $\alpha$-toxin as the staphylococcal effector, and proposes $Z C F 13$ as a candidal factor, in driving synergistic lethality in polymicrobial intra-abdominal infection. Future studies will continue to unravel the complex mechanism of interaction between $C$. albicans and $S$. aureus, ultimately leading to new prophylactic or therapeutic strategies to better manage devastating fungal-bacterial intraabdominal infection. 


\section{LIST OF REFERENCES}

1. Evans AS. Causation and Disease: The Henle-Koch Postulates Revisited. Yale J Biol Med. 1976;49(2):175-95.

2. Todd OA, Peters BM. Candida albicans and Staphylococcus aureus Pathogenicity and Polymicrobial Interactions: Lessons beyond Koch's Postulates. J Fungi (Basel). 2019;5(3). Epub 2019/09/07. doi: 10.3390/jof5030081. PubMed PMID: 31487793; PubMed Central PMCID: PMCPMC6787713.

3. Costerton JW, Lewandowski Z, Caldwell DE, Korber DR, Lappin-Scott HM. Microbial Biofilms. Annu Rev Microbiol. 1995;49(1):711-45. doi: 10.1146/annurev.mi.49.100195.003431.

4. Lloyd-Price J, Abu-Ali G, Huttenhower C. The healthy human microbiome. Genome Med. 2016;8. doi: 10.1186/s13073-016-0307-y.

5. Grice EA, Segre JA. The skin microbiome. Nat Rev Microbiol. 2011;9(4):244-53. doi: 10.1038/nrmicro2537.

6. Braga RM, Dourado MN, Araújo WL. Microbial interactions: ecology in a molecular perspective. Braz J Microbiol. 2016;47 Suppl 1:86-98. doi: 10.1016/j.bjm.2016.10.005.

7. Ghannoum MA, Jurevic RJ, Mukherjee PK, Cui F, Sikaroodi M, Naqvi A, et al. Characterization of the Oral Fungal Microbiome (Mycobiome) in Healthy Individuals. PLoS Pathog. 2010;6(1). doi: 10.1371/journal.ppat.1000713.

8. Sztukowska MN, Dutton LC, Delaney C, Ramsdale M, Ramage G, Jenkinson HF, et al. Community Development between Porphyromonas gingivalis and Candida albicans Mediated by InlJ and Als3. mBio. 2018;9(2). doi: 10.1128/mBio.0020218.

9. Bartnicka D, Karkowska-Kuleta J, Zawrotniak M, Satała D, Michalik K, Zielinska G, et al. Adhesive protein-mediated cross-talk between Candida albicans and Porphyromonas gingivalis in dual species biofilm protects the anaerobic bacterium in unfavorable oxic environment. Sci Rep. 2019;9. doi: 10.1038/s41598-019-40771-8.

10. Ellepola K, Liu Y, Cao T, Koo H, Seneviratne CJ. Bacterial GtfB Augments Candida albicans Accumulation in Cross-Kingdom Biofilms. J Dent Res.2017;96(10):1129-35. doi: 10.1177/0022034517714414.

11. Raja M, Hannan A, Ali K. Association of oral candidal carriage with dental caries in children. Caries Res. 2010;44(3):272-6. doi: 10.1159/000314675.

12. Metwalli KH, Khan SA, Krom BP, Jabra-Rizk MA. Streptococcus mutans, Candida albicans, and the human mouth: a sticky situation. PLoS Pathog. 2013;9(10):e1003616-e. doi: 10.1371/journal.ppat.1003616.

13. Klinke T, Guggenheim B, Klimm W, Thurnheer T. Dental caries in rats associated with Candida albicans. Caries Res. 2011;45(2):100-6. doi: $10.1159 / 000324809$.

14. Kousser C, Clark C, Sherrington S, Voelz K, Hall RA. Pseudomonas aeruginosa inhibits Rhizopus microsporus germination through sequestration of free environmental iron. Sci Rep. 2019;9(1):5714. doi: 10.1038/s41598-019-42175-0. 
15. Morales DK, Jacobs NJ, Rajamani S, Krishnamurthy M, Cubillos-Ruiz JR, Hogan DA. Antifungal mechanisms by which a novel Pseudomonas aeruginosa phenazine toxin kills Candida albicans in biofilms. Mol Microbiol. 2010;78(6):1379-92. doi: 10.1111/j.1365-2958.2010.07414.x.

16. Hogan DA, Kolter R. Pseudomonas-Candida Interactions: An Ecological Role for Virulence Factors. Science. 2002;296(5576):2229-32. doi:

10.1126/science.1070784.

17. Abisado RG, Benomar S, Klaus JR, Dandekar AA, Chandler JR. Bacterial Quorum Sensing and Microbial Community Interactions. mBio. 2018;9(3):e02331-17. doi: 10.1128/mBio.02331-17.

18. Ji G, Beavis R, Novick RP. Bacterial Interference Caused by Autoinducing Peptide Variants. Science. 1997;276(5321):2027-30. doi:

10.1126/science.276.5321.2027.

19. Wellington S, Greenberg EP. Quorum Sensing Signal Selectivity and the Potential for Interspecies Cross Talk. mBio. 2019;10(2):e00146-19. doi:

10.1128/mBio.00146-19.

20. Madhani HD. Quorum Sensing in Fungi: Q\&A. PLoS Pathog. 2011;7(10). doi: 10.1371/journal.ppat.1002301.

21. Hornby JM, Jensen EC, Lisec AD, Tasto JJ, Jahnke B, Shoemaker R, et al. Quorum Sensing in the Dimorphic Fungus Candida albicans Is Mediated by Farnesol. Appl Environ Microbiol. 2001;67(7):2982-92. doi: 10.1128/AEM.67.7.2982-2992.2001.

22. Ramage G, Saville SP, Wickes BL, López-Ribot JL. Inhibition of Candida albicans biofilm formation by farnesol, a quorum-sensing molecule. Appl Environ Microbiol. 2002;68(11):5459-63. doi: 10.1128/aem.68.11.54595463.2002.

23. De Sordi L, Mühlschlegel FA. Quorum sensing and fungal-bacterial interactions in Candida albicans: a communicative network regulating microbial coexistence and virulence. FEMS Yeast Res. 2009;9(7):990-9. doi: 10.1111/j.15671364.2009.00573.x.

24. Togashi N, Inoue Y, Hamashima H, Takano A. Effects of two terpene alcohols on the antibacterial activity and the mode of action of farnesol against Staphylococcus aureus. Molecules. 2008;13(12):3069-76. doi: 10.3390/molecules13123069.

25. Cugini C, Calfee MW, Farrow JM, Morales DK, Pesci EC, Hogan DA. Farnesol, a common sesquiterpene, inhibits PQS production in Pseudomonas aeruginosa. Mol Microbiol. 2007;65(4):896-906. doi: 10.1111/j.1365-2958.2007.05840.x.

26. Kuroda M, Nagasaki S, Ohta T. Sesquiterpene farnesol inhibits recycling of the C55 lipid carrier of the murein monomer precursor contributing to increased susceptibility to beta-lactams in methicillin-resistant Staphylococcus aureus. J Antimicrob Chemother. 2007;59(3):425-32. doi: 10.1093/jac/dk1519.

27. Jabra-Rizk MA, Meiller TF, James CE, Shirtliff ME. Effect of farnesol on Staphylococcus aureus biofilm formation and antimicrobial susceptibility. Antimicrob Agents Chemother. 2006;50(4):1463-9. doi: 10.1128/AAC.50.4.14631469.2006. 
28. Vila T, Kong EF, Ibrahim A, Piepenbrink K, Shetty AC, McCracken C, et al. Candida albicans quorum-sensing molecule farnesol modulates staphyloxanthin production and activates the thiol-based oxidative-stress response in Staphylococcus aureus. Virulence. 2019;10(1):625-42. doi: 10.1080/21505594.2019.1635418.

29. Albuquerque P, Casadevall A. Quorum sensing in fungi--a review. Med Mycol. 2012;50(4):337-45. doi: 10.3109/13693786.2011.652201.

30. Berube BJ, Bubeck Wardenburg J. Staphylococcus aureus $\alpha$-Toxin: Nearly a Century of Intrigue. Toxins. 2013;5(6):1140-66. doi: 10.3390/toxins5061140.

31. Somerville GA, Proctor RA. At the Crossroads of Bacterial Metabolism and Virulence Factor Synthesis in Staphylococci. Microbiol Mol Biol Rev : MMBR. 2009;73(2):233-48. doi: 10.1128/MMBR.00005-09.

32. Thoendel M, Kavanaugh JS, Flack CE, Horswill AR. Peptide signaling in the Staphylococci. Chem Rev. 2011;111(1):117-51. doi: 10.1021/cr100370n.

33. Mayer FL, Wilson D, Hube B. Candida albicans pathogenicity mechanisms. Virulence. 2013;4(2):119-28. doi: 10.4161/viru.22913.

34. Naglik JR, Richardson JP, Moyes DL. Candida albicans Pathogenicity and Epithelial Immunity. PLoS Pathog. 2014;10(8). doi: 10.1371/journal.ppat.1004257.

35. Peters BM, Yano J, Noverr MC, Fidel JPL. Candida vaginitis: when opportunism knocks, the host responds. PLoS Pathog. 2014;10(4):e1003965-e. doi: 10.1371/journal.ppat.1003965.

36. Tong SYC, Davis JS, Eichenberger E, Holland TL, Fowler VG. Staphylococcus aureus infections: epidemiology, pathophysiology, clinical manifestations, and management. Clin Microbiol Rev. 2015;28(3):603-61. doi: 10.1128/CMR.0013414.

37. Monroy TB, Maldonado VM, Martínez FF, Barrios BA, Vargas LOS. Colonización por Candida albicans, Staphylococcus aureus y Streptococcus mutans en pacientes por- tadores de prótesis dentales Candida albicans, Staphylococcus aureus and Streptococcus mutans colonization in patients wearing dental prosthesis. Med Oral Patol Oral Cir Bucal.13.

38. Liesenborghs L, Verhamme P, Vanassche T. Staphylococcus aureus, master manipulator of the human hemostatic system. J Thromb Haemost.

2018;16(3):441-54. doi: 10.1111/jth.13928.

39. Pettit RK, Weber CA, Lawrence SB, Pettit GR, Kean MJ, Cage GD. In vivo activity of anprocide alone, and in vitro activity in combination with conventional antibiotics against Staphylococcus aureus and Staphylococcus epidermidis biofilms. J Med Microbiol. 2009;58(Pt 9):1203-6. doi: 10.1099/jmm.0.008268-0.

40. Nanra JS, Buitrago SM, Crawford S, Ng J, Fink PS, Hawkins J, et al. Capsular polysaccharides are an important immune evasion mechanism for Staphylococcus aureus. Hum Vaccin Immunother. 2013;9(3):480-7. doi: 10.4161/hv.23223.

41. Valenza G, Tappe D, Turnwald D, Frosch M, König C, Hebestreit H, et al. Prevalence and antimicrobial susceptibility of microorganisms isolated from sputa of patients with cystic fibrosis. J Cyst Fibros. 2008;7(2):123-7. doi:

10.1016/j.jcf.2007.06.006. 
42. Pate JC, Jones DB, Wilhelmus KR. Prevalence and spectrum of bacterial coinfection during fungal keratitis. Brit J Opthalmol. 2006;90(3):289-92. doi: 10.1136/bjo.2005.081869.

43. Tawara Y, Honma K, Naito Y. Methicillin-resistant Staphylococcus aureus and Candida albicans on denture surfaces. Bull Tokyo Dent Coll. 1996;37(3):119-28.

44. Gupta N, Haque AKMF, Mukhopadhyay G, Narayan R, Prasad RK. Interactions between bacteria and Candida in the burn wound. Burns. 2005.

45. Shirtliff ME, Peters BM, Jabra-Rizk MA. Cross-kingdom interactions: Candida albicans and bacteria. FEMS Microbiol Lett. 2009;299(1):1-8. doi: 10.1111/j.1574-6968.2009.01668.x.

46. Harriott MM, Noverr MC. Candida albicans and Staphylococcus aureus form polymicrobial biofilms: effects on antimicrobial resistance. Antimicrob Agents Chemoth. 2009;53(9):3914-22. doi: 10.1128/AAC.00657-09.

47. Peters BM, Jabra-Rizk MA, Scheper MA, Leid JG, Costerton JW, Shirtliff ME. Microbial interactions and differential protein expression in Staphylococcus aureus -Candida albicans dual-species biofilms. FEMS Immunol Med Microbiol. 2010;59(3):493-503. doi: 10.1111/j.1574-695X.2010.00710.x.

48. Harriott MM, Noverr MC. Ability of Candida albicans Mutants To Induce Staphylococcus aureus Vancomycin Resistance during Polymicrobial Biofilm Formation. Antimicrob Agents Chemoth. 2010;54(9):3746-55. doi: 10.1128/AAC.00573-10.

49. Kong EF, Tsui C, Kucharíková S, Andes D, Van Dijck P, Jabra-Rizk MA. Commensal Protection of Staphylococcus aureus against Antimicrobials by Candida albicans Biofilm Matrix. mBio. 2016;7(5). doi: 10.1128/mBio.01365-16.

50. Peters BM, Ovchinnikova ES, Krom BP, Schlecht LM, Zhou H, Hoyer LL, et al. Staphylococcus aureus adherence to Candida albicans hyphae is mediated by the hyphal adhesin Als3p. Microbiology. 2012;158(Pt 12):2975-86. doi: 10.1099/mic.0.062109-0.

51. Schlecht LM, Peters BM, Krom BP, Freiberg JA, Hänsch GM, Filler SG, et al. Systemic Staphylococcus aureus infection mediated by Candida albicans hyphal invasion of mucosal tissue. Microbiology. 2015;161(Pt 1):168-81. doi: 10.1099/mic.0.083485-0.

52. Kong EF, Kucharíková S, Van Dijck P, Peters BM, Shirtliff ME, Jabra-Rizk MA. Clinical implications of oral candidiasis: host tissue damage and disseminated bacterial disease. Infect Immun. 2015;83(2):604-13. doi: 10.1128/IAI.02843-14.

53. Baena-Monroy T, Moreno-Maldonado V, Franco-Martínez F, Aldape-Barrios B, Quindós G, Sánchez-Vargas LO. Candida albicans, Staphylococcus aureus and Streptococcus mutans colonization in patients wearing dental prosthesis. Medicina oral, patologia oral y cirugia bucal. 2005;10 Suppl 1:E27-39.

54. Santos SG, Serufo JC, Silva RAP, Marra BA, Reis CMF, Hamdan JS, et al. Microbiologic profile of intra-abdominal infections at Belo Horizonte, Brazil. American J Infect Contr. 2003;31(3):135-43. doi: 10.1067/mic.2003.54.

55. Lopez N, Kobayashi L, Coimbra R. A Comprehensive review of abdominal infections. World J Emerg Surg. 2011;6:7. doi: 10.1186/1749-7922-6-7.

56. de Ruiter J, Weel J, Manusama E, Kingma WP, van der Voort PHJ. The Epidemiology of Intra-Abdominal Flora in Critically Ill Patients with Secondary 
and Tertiary Abdominal Sepsis. Infection. 2009;37(6):522. doi: 10.1007/s15010009-8249-6.

57. McNeil JC, Vallejo JG, Hultén KG, Kaplan SL. Osteoarticular Infections Following Open or Penetrating Trauma in Children in the Post-CommunityAcquired Methicillin-resistant Staphylococcus aureus Era: The Impact of Enterobacter cloacae. Pediatr Infect Dis J. 2018;37(12):1204-10. doi: 10.1097/INF.0000000000001991. PubMed PMID: 29570590.

58. Powers ME, Becker REN, Sailer A, Turner JR, Wardenburg JB. Synergistic Action of Staphylococcus aureus $\alpha$-Toxin on Platelets and Myeloid Lineage Cells Contributes to Lethal Sepsis. Cell Host Microbe. 2015;17(6):775-87. doi:10.1016/j.chom.2015.05.011.

59. Carlson E. Synergistic effect of Candida albicans and Staphylococcus aureus on mouse mortality. Infect Immun 1982;38(3):921-4.

60. Carlson E. Enhancement by Candida albicans of Staphylococcus aureus, Serratia marcescens, and Streptococcus faecalis in the establishment of infection in mice. Infect Immun. 1983;39(1):193-7.

61. Carlson E, Johnson G. Protection by Candida albicans of Staphylococcus aureus in the establishment of dual infection in mice. Infect Immun. 1985;50(3):655-9.

62. Peters BM, Noverr MC. Candida albicans-Staphylococcus aureus Polymicrobial Peritonitis Modulates Host Innate Immunity. Infect Immun. 2013;81(6):2178-89. doi: 10.1128/IAI.00265-13.

63. Durkin JP, Shier WT. Staphylococcal delta toxin stimulates endogenous phospholipase A2 activity and prostaglandin synthesis in fibroblasts. Biochim Biophys Acta. 1981;663(2):467-79. doi: 10.1016/0005-2760(81)90175-2.

64. Raya SA, Trembovler V, Shohami E, Lazarovici P. Cytolysins increase intracellular calcium and induce eicosanoids release by pheochromocytoma PC12 cell cultures. Nat Toxins. 1993;1(5):263-70. doi: 10.1002/nt.2620010502.

65. Todd OA, Fidel JPL, Harro JM, Hilliard JJ, Tkaczyk C, Sellman BR, et al. Candida albicans Augments Staphylococcus aureus Virulence by Engaging the Staphylococcal agr Quorum Sensing System. mBio. 2019;10(3). doi: 10.1128/mBio.00910-19.

66. Nash EE, Peters BM, Palmer GE, Fidel PL, Noverr MC. Morphogenesis Is Not Required for Candida albicans-Staphylococcus aureus Intra-Abdominal Infection-Mediated Dissemination and Lethal Sepsis. Infect Immun. 2014;82(8):3426-35. doi: 10.1128/IAI.01746-14.

67. Nash EE, Peters BM, Fidel PL, Noverr MC. Morphology-Independent Virulence of Candida Species during Polymicrobial Intra-abdominal Infections with Staphylococcus aureus. Infect Immun. 2016;84(1):90-8. doi: 10.1128/IAI.0105915.

68. Cheng S, Clancy CJ, Xu W, Schneider F, Hao B, Mitchell AP, et al. Profiling of Candida albicans Gene Expression During Intra-abdominal Candidiasis Identifies Biologic Processes Involved in Pathogenesis. J Infect Dis. 2013;208(9):1529-37. doi: 10.1093/infdis/jit335.

69. De CSS, Fortaleza CM, Witzel CL, Silveira M, Bonesso MF, Marques SA, et al. Toxigenic profile of methicillin-sensitive and resistant Staphylococcus aureus 
isolated from special groups. Ann Clin Microbiol Antimicrob. 2016;15:9-. doi: 10.1186/s12941-016-0125-5.

70. Xie Y, He Y, Gehring A, Hu Y, Li Q, Tu S-I, et al. Genotypes and toxin gene profiles of Staphylococcus aureus clinical isolates from China. PloS ONE. 2011;6(12):e28276. doi: 10.1371/journal.pone.0028276.

71. Carlson E. Effect of strain of Staphylococcus aureus on synergism with Candida albicans resulting in mouse mortality and morbidity. Infect Immun 1983;42(1):285-92.

72. Carlson EC. Synergism of Candida albicans and delta toxin producing Staphylococcus aureus on mouse mortality and morbidity: protection by indomethacin. Zentralblatt fur Bakteriologie, Mikrobiologie, und Hygiene Series A, Medical microbiology, infectious diseases, virology, parasitology. 1988;269(3):377-86.

73. David MZ, Daum RS. Community-Associated Methicillin-Resistant Staphylococcus aureus: Epidemiology and Clinical Consequences of an Emerging Epidemic. Clin Microbiol Rev. 2010;23(3):616-87. doi: 10.1128/CMR.00081-09.

74. Kim J, Sudbery P. Candida albicans, a major human fungal pathogen. J Microbiol. 2011;49(2):171. doi: 10.1007/s12275-011-1064-7.

75. Carey AJ, Saiman L, Polin RA. Hospital-acquired infections in the NICU: epidemiology for the new millennium. Clin Perinatol. 2008;35(1):223-49, x. Epub 2008/02/19. doi: 10.1016/j.clp.2007.11.014. PubMed PMID: 18280884.

76. Klotz SA, Chasin BS, Powell B, Gaur NK, Lipke PN. Polymicrobial bloodstream infections involving Candida species: analysis of patients and review of the literature. Diagn Micro Infec Dis. 2007;59(4):401-6. doi: 10.1016/j.diagmicrobio.2007.07.001.

77. Peters BM, Jabra-Rizk MA, O'May GA, Costerton JW, Shirtliff ME. Polymicrobial Interactions: Impact on Pathogenesis and Human Disease. Clin Microbiol Rev. 2012;25(1):193-213. doi: 10.1128/CMR.00013-11.

78. Gordon RJ, Lowy FD. Pathogenesis of Methicillin-Resistant Staphylococcus aureus Infection. Clin Infect Dis. 2008;46(Suppl 5):S350-S9. doi: $10.1086 / 533591$.

79. Le KY, Otto M. Quorum-sensing regulation in staphylococci-an overview. Front Microbiol. 2015;6. doi: 10.3389/fmicb.2015.01174.

80. Janzon L, Arvidson S. The role of the delta-lysin gene $(h l d)$ in the regulation of virulence genes by the accessory gene regulator (agr) in Staphylococcus aureus. The EMBO Journal. 1990;9(5):1391-9.

81. Craven RR, Gao X, Allen IC, Gris D, Wardenburg JB, McElvania-TeKippe E, et al. Staphylococcus aureus $\alpha$-Hemolysin Activates the NLRP3-Inflammasome in Human and Mouse Monocytic Cells. PLoS ONE. 2009;4(10). doi: 10.1371/journal.pone.0007446.

82. Hilliard JJ, Datta V, Tkaczyk C, Hamilton M, Sadowska A, Jones-Nelson O, et al. Anti-Alpha-Toxin Monoclonal Antibody and Antibiotic Combination Therapy Improves Disease Outcome and Accelerates Healing in a Staphylococcus aureus Dermonecrosis Model. Antimicrob Agents Chemoth. 2015;59(1):299-309. doi: 10.1128/AAC.03918-14. 
83. Schmitz F-J, Veldkamp K-E, Kessel KPMV, Verhoef J, Strijp JAGV. DeltaToxin from Staphylococcus aureus as a Costimulator of Human Neutrophil Oxidative Burst. J Infect Dis. 1997;176(6):1531-7. doi: 10.1086/514152.

84. Tkaczyk C, Hamilton MM, Datta V, Yang XP, Hilliard JJ, Stephens GL, et al. Staphylococcus aureus Alpha Toxin Suppresses Effective Innate and Adaptive Immune Responses in a Murine Dermonecrosis Model. PLoS ONE. 2013;8(10):e75103. doi: 10.1371/journal.pone.0075103.

85. Walker B, Krishnasastry M, Zorn L, Kasianowicz J, Bayley H. Functional expression of the alpha-hemolysin of Staphylococcus aureus in intact Escherichia coli and in cell lysates. Deletion of five C-terminal amino acids selectively impairs hemolytic activity. J Bio Chem. 1992;267(15):10902-9.

86. Adhikari RP, Ajao AO, Aman MJ, Karauzum H, Sarwar J, Lydecker AD, et al. Lower Antibody Levels to Staphylococcus aureus Exotoxins Are Associated With Sepsis in Hospitalized Adults With Invasive S. aureus Infections. J Infect Dis. 2012;206(6):915-23. doi: 10.1093/infdis/jis462.

87. Ikeh MAC, Fidel PL, Noverr MC. Identification of Specific Components of the Eicosanoid Biosynthetic and Signaling Pathway Involved in Pathological Inflammation during Intra-abdominal Infection with Candida albicans and Staphylococcus aureus. Infect Immun. 2018;86(7). doi: 10.1128/IAI.00144-18.

88. Fink D, Contreras ML, Lelkes PI, Lazarovici P. Staphylococcus aureus alphatoxin activates phospholipases and induces a Ca2+ influx in PC12 cells. Cell Signal. 1989;1(4):387-93.

89. Fey PD, Endres JL, Yajjala VK, Widhelm TJ, Boissy RJ, Bose JL, et al. A genetic resource for rapid and comprehensive phenotype screening of nonessential Staphylococcus aureus genes. mBio. 2013;4(1):e00537-12. Epub 2013/02/14. doi: mBio.00537-12 [pii]10.1128/mBio.00537-12. PubMed PMID: 23404398; PubMed Central PMCID: PMC3573662.

90. Yarwood JM, Schlievert PM. Quorum sensing in Staphylococcus infections. J Clin Invest. 2003;112(11):1620-5. doi: 10.1172/jci200320442.

91. Monk IR, Tree JJ, Howden BP, Stinear TP, Foster TJ. Complete Bypass of Restriction Systems for Major Staphylococcus aureus Lineages. mBio. 2015;6(3):e00308-15. Epub 2015/05/28. doi: mBio.00308-15 [pii]10.1128/mBio.00308-15. PubMed PMID: 26015493; PubMed Central PMCID: PMC4447248.

92. Donegan K, Matyac C, Seidler R, Porteous A. Evaluation of methods for sampling, recovery, and enumeration of bacteria applied to the phylloplane. Appl Environ Microbiol. 1991;57(1):51-6. Epub 1991/01/01. PubMed PMID: 16348404; PubMed Central PMCID: PMC182663.

93. Adam B, Baillie GS, Douglas LJ. Mixed species biofilms of Candida albicans and Staphylococcus epidermidis. J Med Microbiol. 2002;51(4):344-9. Epub 2002/04/03. PubMed PMID: 11926741.

94. Peters BM, Ward RM, Rane HS, Lee SA, Noverr MC. Efficacy of ethanol against Candida albicans and Staphylococcus aureus polymicrobial biofilms. Antimicrob Agents Chemoth. 2013;57(1):74-82. Epub 2012/10/17. doi: AAC.01599-12 [pii]10.1128/AAC.01599-12. PubMed PMID: 23070170; PubMed Central PMCID: PMC3535989. 
95. Beenken KE, Dunman PM, McAleese F, Macapagal D, Murphy E, Projan SJ, et al. Global gene expression in Staphylococcus aureus biofilms. J Bacteriol. 2004;186(14):4665-84. Epub 2004/07/03. doi: 10.1128/JB.186.14.46654684.2004186/14/4665 [pii]. PubMed PMID: 15231800; PubMed Central PMCID: PMC438561.

96. Livak KJ, Schmittgen TD. Analysis of relative gene expression data using realtime quantitative PCR and the 2(-Delta Delta C(T)) Method. Methods. 2001;25(4):402-8. Epub 2002/02/16. doi: 10.1006/meth.2001.1262 S10462023(01)91262-9 [pii]. PubMed PMID: 11846609.

97. Rauch S, DeDent AC, Kim HK, Bubeck Wardenburg J, Missiakas DM, Schneewind O. Abscess Formation and Alpha-Hemolysin Induced Toxicity in a Mouse Model of Staphylococcus aureus Peritoneal Infection. Infect Immun. 2012;80(10):3721-32. doi: 10.1128/IAI.00442-12.

98. Bhakdi S, Tranum-Jensen J. Alpha-toxin of Staphylococcus aureus. Microbiol Rev. 1991;55(4):733-51.

99. Cheung AL, Eberhardt K, Heinrichs JH. Regulation of protein A synthesis by the sar and agr loci of Staphylococcus aureus. Infect Immun.1997;65(6):2243-9.

100. Chatterjee SS, Chen L, Joo H-S, Cheung GYC, Kreiswirth BN, Otto M. Distribution and Regulation of the Mobile Genetic Element-Encoded PhenolSoluble Modulin PSM-mec in Methicillin-Resistant Staphylococcus aureus. PLoS ONE. 2011;6(12):e28781. doi: 10.1371/journal.pone.0028781.

101. Spaulding AR, Satterwhite EA, Lin Y-C, Chuang-Smith ON, Frank KL, Merriman JA, et al. Comparison of Staphylococcus aureus strains for ability to cause infective endocarditis and lethal sepsis in rabbits. Front Cell Infect Mi. 2012;2. doi: 10.3389/fcimb.2012.00018.

102. Bose JL, Daly SM, Hall PR, Bayles KW. Identification of the Staphylococcus aureus vfrAB Operon, a Novel Virulence Factor Regulatory Locus. Infect Immun. 2014;82(5):1813-22. doi: 10.1128/IAI.01655-13.

103. Sharma-Kuinkel BK, Mongodin EF, Myers JR, Vore KL, Canfield GS, Fraser CM, et al. Potential Influence of Staphylococcus aureus Clonal Complex 30 Genotype and Transcriptome on Hematogenous Infections. Open Forum Infect Dis. 2015;2(3). doi: 10.1093/ofid/ofv093.

104. Xiong YQ, Willard J, Yeaman MR, Cheung AL, Bayer AS. Regulation of Staphylococcus aureus alpha-toxin gene (hla) expression by agr, sarA, and sae in vitro and in experimental infective endocarditis. J Infect Dis. 2006;194(9):126775. Epub 2006/10/17. doi: JID36622 [pii]10.1086/508210. PubMed PMID: 17041853.

105. Goerke C, Fluckiger U, Steinhuber A, Zimmerli W, Wolz C. Impact of the regulatory loci agr, sarA and sae of Staphylococcus aureus on the induction of alpha-toxin during device-related infection resolved by direct quantitative transcript analysis. Mol Microbiol. 2001;40(6):1439-47. Epub 2001/07/10. doi: mmi2494 [pii]. PubMed PMID: 11442841.

106. Paulander W, Nissen Varming A, Baek KT, Haaber J, Frees D, Ingmer H. Antibiotic-mediated selection of quorum-sensing-negative Staphylococcus aureus. mBio. 2013;3(6):e00459-12. Epub 2012/11/13. doi: mBio.00459-12 
[pii]10.1128/mBio.00459-12. PubMed PMID: 23143800; PubMed Central PMCID: PMC3509436.

107. Oganesyan V, Peng L, Damschroder MM, Cheng L, Sadowska A, Tkaczyk C, et al. Mechanisms of neutralization of a human anti-alpha-toxin antibody. J Biol Chem. 2014;289(43):29874-80. Epub 2014/09/12. doi: M114.601328 [pii] 10.1074/jbc.M114.601328. PubMed PMID: 25210036; PubMed Central PMCID: PMC4207998.

108. Diep BA, Hilliard JJ, Le VT, Tkaczyk C, Le HN, Tran VG, et al. Targeting Alpha Toxin To Mitigate Its Lethal Toxicity in Ferret and Rabbit Models of Staphylococcus aureus Necrotizing Pneumonia. Antimicrob Agents Chemother. 2017;61(4). Epub 2017/01/25. doi: AAC.02456-16 [pii]10.1128/AAC.02456-16. PubMed PMID: 28115346; PubMed Central PMCID: PMC5365647.

109. Hua L, Cohen TS, Shi Y, Datta V, Hilliard JJ, Tkaczyk C, et al. MEDI4893* Promotes Survival and Extends the Antibiotic Treatment Window in a Staphylococcus aureus Immunocompromised Pneumonia Model. Antimicrob Agents Chemother. 2015;59(8):4526-32. Epub 2015/05/20. doi: 10.1128/AAC.00510-15. PubMed PMID: 25987629; PubMed Central PMCID: PMCPMC4505239.

110. Tkaczyk C, Hamilton MM, Sadowska A, Shi Y, Chang CS, Chowdhury P, et al. Targeting Alpha Toxin and ClfA with a Multimechanistic Monoclonal-AntibodyBased Approach for Prophylaxis of Serious Staphylococcus aureus Disease. mBio. 2016;7(3). Epub 2016/06/30. doi: 10.1128/mBio.00528-16. PubMed PMID: 27353753; PubMed Central PMCID: PMCPMC4937210.

111. Cohen TS, Hilliard JJ, Jones-Nelson O, Keller AE, O'Day T, Tkaczyk C, et al. Staphylococcus aureus alpha toxin potentiates opportunistic bacterial lung infections. Sci Transl Med. 2016;8(329):329ra31. Epub 2016/03/11. doi: 8/329/329ra31 [pii] 10.1126/scitranslmed.aad9922. PubMed PMID: 26962155.

112. Rappuoli R, Montecucco C. Guidebook to protein toxins and their use in cell biology. Oxford ; New York: Oxford University Press; 1997. xviii, 256 p. p.

113. Bamford CV, d'Mello A, Nobbs AH, Dutton LC, Vickerman MM, Jenkinson HF. Streptococcus gordonii modulates Candida albicans biofilm formation through intergeneric communication. Infect Immun. 2009;77(9):3696-704. Epub 2009/06/17. doi: IAI.00438-09 [pii]10.1128/IAI.00438-09. PubMed PMID: 19528215 ; PubMed Central PMCID: PMC2737996.

114. Hogan DA, Vik A, Kolter R. A Pseudomonas aeruginosa quorum-sensing molecule influences Candida albicans morphology. Mol Microbiol. 2004;54(5):1212-23. Epub 2004/11/24. doi: MMI4349 [pii]10.1111/j.13652958.2004.04349.x. PubMed PMID: 15554963.

115. Regassa LB, Novick RP, Betley MJ. Glucose and nonmaintained $\mathrm{pH}$ decrease expression of the accessory gene regulator (agr) in Staphylococcus aureus. Infect Immun. 1992;60(8):3381-8.

116. Pfaller MA, Diekema DJ. Epidemiology of invasive candidiasis: a persistent public health problem. Clin Microbiol Rev. 2007;20(1):133-63. Epub 2007/01/16. doi: 20/1/133 [pii]10.1128/CMR.00029-06. PubMed PMID: 17223626; PubMed Central PMCID: PMC1797637. 
117. Cheng S, Clancy CJ, Hartman DJ, Hao B, Nguyen MH. Candida glabrata intraabdominal candidiasis is characterized by persistence within the peritoneal cavity and abscesses. Infect Immun. 2014;82(7):3015-22. Epub 2014/05/07. doi: IAI.00062-14 [pii] 10.1128/IAI.00062-14. PubMed PMID: 24799629; PubMed Central PMCID: PMC4097615.

118. Klaerner HG, Uknis ME, Acton RD, Dahlberg PS, Carlone-Jambor C, Dunn DL. Candida albicans and Escherichia coli are synergistic pathogens during experimental microbial peritonitis. J Surg Res. 1997;70(2):161-5. Epub 1997/07/01. doi: S0022-4804(97)95110-6 [pii]10.1006/jsre.1997.5110. PubMed PMID: 9245566.

119. Klotz SA, Chasin BS, Powell B, Gaur NK, Lipke PN. Polymicrobial bloodstream infections involving Candida species: analysis of patients and review of the literature. Diagn Microbiol Infect Dis. 2007;59(4):401-6. PubMed PMID: 17888612.

120. Valenza G, Tappe D, Turnwald D, Frosch M, Konig C, Hebestreit H, et al. Prevalence and antimicrobial susceptibility of microorganisms isolated from sputa of patients with cystic fibrosis. J Cyst Fibros. 2008;7(2):123-7. Epub 2007/08/19. doi: S1569-1993(07)00098-7 [pii]10.1016/j.jcf.2007.06.006. PubMed PMID: 17693140.

121. Le KY, Otto M. Quorum-sensing regulation in staphylococci-an overview. Front Microbiol. 2015;6:1174. Epub 2015/11/19. doi: 10.3389/fmicb.2015.01174. PubMed PMID: 26579084; PubMed Central PMCID: PMC4621875.

122. Todd OA, Noverr MC, Peters BM. Candida albicans Impacts Staphylococcus aureus Alpha-Toxin Production via Extracellular Alkalinization. mSphere. 2019;4(6). Epub 2019/11/15. doi: 10.1128/mSphere.00780-19. PubMed PMID: 31722996; PubMed Central PMCID: PMCPMC6854045.

123. Regassa LB, Betley MJ. Alkaline $\mathrm{pH}$ decreases expression of the accessory gene regulator (agr) in Staphylococcus aureus. J Bacteriol. 1992;174(15):5095-100.

124. Vylkova S, Lorenz MC. Modulation of Phagosomal pH by Candida albicans Promotes Hyphal Morphogenesis and Requires Stp2p, a Regulator of Amino Acid Transport. PLoS Pathog. 2014;10(3):e1003995. doi:

10.1371/journal.ppat.1003995.

125. Yarwood JM, Bartels DJ, Volper EM, Greenberg EP. Quorum sensing in Staphylococcus aureus biofilms. J Bacteriol. 2004;186(6):1838-50. Epub 2004/03/05. doi: 10.1128/jb.186.6.1838-1850.2004. PubMed PMID: 14996815; PubMed Central PMCID: PMCPMC355980.

126. Vylkova S, Lorenz MC. Phagosomal Neutralization by the Fungal Pathogen Candida albicans Induces Macrophage Pyroptosis. Infect Immun. 2017;85(2). Epub 2016/11/23. doi: 10.1128/IAI.00832-16. PubMed PMID: 27872238; PubMed Central PMCID: PMCPMC5278172.

127. Vylkova S, Carman AJ, Danhof HA, Collette JR, Zhou H, Lorenz MC. The Fungal Pathogen Candida albicans Autoinduces Hyphal Morphogenesis by Raising Extracellular pH. mBio. 2011;2(3). doi: 10.1128/mBio.00055-11.

128. Danhof HA, Vylkova S, Vesely EM, Ford AE, Gonzalez-Garay M, Lorenz MC. Robust Extracellular $\mathrm{pH}$ Modulation by Candida albicans during Growth in Carboxylic Acids. mBio. 2016;7(6):e01646-16. doi: 10.1128/mBio.01646-16. 
129. Brown AJ, Odds FC, Gow NA. Infection-related gene expression in Candida albicans. Curr Opin Microbiol. 2007;10(4):307-13. Epub 2007/08/21. doi: 10.1016/j.mib.2007.04.001. PubMed PMID: 17707687.

130. Collins EB, Hardt P. Inhibition of Candida albicans by Lactobacillus acidophilus. J Dairy Sci. 1980;63(5):830-2. Epub 1980/05/01. doi: 10.3168/jds.S00220302(80)83013-X. PubMed PMID: 6771309.

131. Canovas J, Baldry M, Bojer MS, Andersen PS, Gless BH, Grzeskowiak PK, et al. Cross-Talk between Staphylococcus aureus and Other Staphylococcal Species via the agr Quorum Sensing System. Front Microbiol. 2016;7. doi: 10.3389/fmicb.2016.01733.

132. Miramon P, Lorenz MC. The SPS amino acid sensor mediates nutrient acquisition and immune evasion in Candida albicans. Cell Microbiol. 2016;18(11):1611-24. Epub 2016/10/26. doi: 10.1111/cmi.12600. PubMed PMID: 27060451; PubMed Central PMCID: PMCPMC5501722.

133. Vergidis P, Clancy CJ, Shields RK, Park SY, Wildfeuer BN, Simmons RL, et al. Intra-Abdominal Candidiasis: The Importance of Early Source Control and Antifungal Treatment. PLoS ONE. 2016;11(4):e0153247. Epub 2016/04/29. doi: 10.1371/journal.pone.0153247. PubMed PMID: 27123857; PubMed Central PMCID: PMCPMC4849645.

134. Azim A, Ahmed A, Baronia AK, Marak RSK, Muzzafar N. INTRAABDOMINAL CANDIDIASIS. Eur Med J. 2017:10.

135. Baliga S, Muglikar S, Kale R. Salivary pH: A diagnostic biomarker. J Indian Soc Periodontol. 2013;17(4):461-5. doi: 10.4103/0972-124X.118317.

136. Kellum JA. Determinants of blood $\mathrm{pH}$ in health and disease. Crit Care. 2000;4(1):6-14. doi: 10.1186/cc644.

137. Godha K, Tucker KM, Biehl C, Archer DF, Mirkin S. Human vaginal pH and microbiota: an update. Gynecological Endocrinology. 2018;34(6):451-5. doi: 10.1080/09513590.2017.1407753.

138. Aoi W, Marunaka Y. Importance of $\mathrm{pH}$ Homeostasis in Metabolic Health and Diseases: Crucial Role of Membrane Proton Transport. BioMed Res Intern. 2014;2014. doi: 10.1155/2014/598986.

139. Cuesta AI, Jewtuchowicz V, Brusca MI, Nastri ML, Rosa AC. Prevalence of Staphylococcus spp and Candida spp in the oral cavity and periodontal pockets of periodontal disease patients. Acta Odontol Latinoam. 2010;23(1):20-6. Epub 2010/07/22. PubMed PMID: 20645638.

140. Montravers P, Dupont H, Gauzit R, Veber B, Auboyer C, Blin P, et al. Candida as a risk factor for mortality in peritonitis. Crit Care Med. 2006;34(3):646-52. Epub 2006/03/01. doi: 10.1097/01.CCM.0000201889.39443.D2. PubMed PMID: 16505648.

141. Hildebrand A, Pohl M, Bhakdi S. Staphylococcus aureus alpha-toxin. Dual mechanism of binding to target cells. J Biol Chem. 1991;266(26):17195-200. Epub 1991/09/15. PubMed PMID: 1894613.

142. Surewaard BGJ, Thanabalasuriar A, Zeng Z, Tkaczyk C, Cohen TS, Bardoel BW, et al. alpha-Toxin Induces Platelet Aggregation and Liver Injury during Staphylococcus aureus Sepsis. Cell Host Microbe. 2018;24(2):271-84 e3. Epub 
2018/07/24. doi: 10.1016/j.chom.2018.06.017. PubMed PMID: 30033122;

PubMed Central PMCID: PMCPMC6295203.

143. Homann OR, Dea J, Noble SM, Johnson AD. A phenotypic profile of the Candida albicans regulatory network. PLoS Genet. 2009;5(12):e1000783. Epub 2009/12/31. doi: 10.1371/journal.pgen.1000783. PubMed PMID: 20041210; PubMed Central PMCID: PMCPMC2790342.

144. Monk IR, Shah IM, Xu M, Tan MW, Foster TJ. Transforming the untransformable: application of direct transformation to manipulate genetically Staphylococcus aureus and Staphylococcus epidermidis. mBio. 2012;3(2). Epub 2012/03/22. doi: 10.1128/mBio.00277-11. PubMed PMID: 22434850; PubMed Central PMCID: PMCPMC3312211.

145. Czako R, Vogel L, Lamirande EW, Bock KW, Moore IN, Ellebedy AH, et al. In Vivo Imaging of Influenza Virus Infection in Immunized Mice. mBio. 2017;8(3). Epub 2017/06/01. doi: 10.1128/mBio.00714-17. PubMed PMID: 28559489; PubMed Central PMCID: PMCPMC5449660.

146. Reuss O, Vik A, Kolter R, Morschhauser J. The SAT1 flipper, an optimized tool for gene disruption in Candida albicans. Gene. 2004;341:119-27. Epub 2004/10/12. doi: S0378111904003555 [pii]10.1016/j.gene.2004.06.021. PubMed PMID: 15474295.

147. Gietz D, St Jean A, Woods RA, Schiestl RH. Improved method for high efficiency transformation of intact yeast cells. Nucleic Acids Res. 1992;20(6):1425. Epub 1992/03/25. doi: 10.1093/nar/20.6.1425. PubMed PMID: 1561104; PubMed Central PMCID: PMCPMC312198.

148. Znaidi S, Nesseir A, Chauvel M, Rossignol T, d'Enfert C. A Comprehensive Functional Portrait of Two Heat Shock Factor-Type Transcriptional Regulators Involved in Candida albicans Morphogenesis and Virulence. PLoS Pathog. 2013;9(8):e1003519. doi: 10.1371/journal.ppat.1003519.

149. Valeva A, Palmer M, Hilgert K, Kehoe M, Bhakdi S. Correct oligomerization is a prerequisite for insertion of the central molecular domain of staphylococcal alphatoxin into the lipid bilayer. Biochim Biophys Acta. 1995;1236(2):213-8. Epub 1995/06/14. doi: 10.1016/0005-2736(95)00060-g. PubMed PMID: 7794960.

150. Valeva A, Pongs J, Bhakdi S, Palmer M. Staphylococcal alpha-toxin: the role of the $\mathrm{N}$-terminus in formation of the heptameric pore -- a fluorescence study. Biochim Biophys Acta. 1997;1325(2):281-6. Epub 1997/04/26. doi: 10.1016/s0005-2736(96)00266-0. PubMed PMID: 9168153.

151. Valeva A, Palmer M, Bhakdi S. Staphylococcal alpha-toxin: formation of the heptameric pore is partially cooperative and proceeds through multiple intermediate stages. Biochemistry. 1997;36(43):13298-304. Epub 1997/10/28. doi: 10.1021/bi971075r. PubMed PMID: 9341221.

152. Valeva A, Weisser A, Walker B, Kehoe M, Bayley H, Bhakdi S, et al. Molecular architecture of a toxin pore: a 15-residue sequence lines the transmembrane channel of staphylococcal alpha-toxin. EMBO J. 1996;15(8):1857-64. Epub 1996/04/15. PubMed PMID: 8617232; PubMed Central PMCID: PMCPMC450103.

153. Bauer J, Wendland J. Candida albicans Sfl1 suppresses flocculation and filamentation. Eukaryot Cell. 2007;6(10):1736-44. Epub 2007/09/04. doi: 
10.1128/EC.00236-07. PubMed PMID: 17766464; PubMed Central PMCID: PMCPMC2043394.

154. Li Y, Su C, Mao X, Cao F, Chen J. Roles of Candida albicans Sfl1 in hyphal development. Eukaryot Cell. 2007;6(11):2112-21. Epub 2007/08/24. doi: 10.1128/EC.00199-07. PubMed PMID: 17715361; PubMed Central PMCID: PMCPMC2168412.

155. Vandeputte P, Ischer F, Sanglard D, Coste AT. In vivo systematic analysis of Candida albicans Zn2-Cys6 transcription factors mutants for mice organ colonization. PLoS ONE. 2011;6(10):e26962. Epub 2011/11/11. doi: 10.1371/journal.pone.0026962. PubMed PMID: 22073120; PubMed Central PMCID: PMCPMC3205040.

156. Pierrehumbert A, Ischer F, Coste AT. Unexpected Transcripts in Tn7 orf19.2646 C. albicans Mutant Lead to Low Fungal Burden Phenotype In vivo. Front Microbiol. 2017;8:873. Epub 2017/06/01. doi: 10.3389/fmicb.2017.00873. PubMed PMID: 28559890; PubMed Central PMCID: PMCPMC5432668.

157. Wangsanut T, Ghosh AK, Metzger PG, Fonzi WA, Rolfes RJ. Grf10 and Bas1 Regulate Transcription of Adenylate and One-Carbon Biosynthesis Genes and Affect Virulence in the Human Fungal Pathogen Candida albicans. mSphere. 2017;2(4). Epub 2017/08/05. doi: 10.1128/mSphere.00161-17. PubMed PMID: 28776040; PubMed Central PMCID: PMCPMC5541157.

158. Nobile CJ, Mitchell AP. Regulation of cell-surface genes and biofilm formation by the $C$. albicans transcription factor Bcr1p. Curr Biol. 2005;15(12):1150-5. Epub 2005/06/21. doi: 10.1016/j.cub.2005.05.047. PubMed PMID: 15964282.

159. Romanowski K, Zaborin A, Valuckaite V, Rolfes RJ, Babrowski T, Bethel C, et al. Candida albicans isolates from the gut of critically ill patients respond to phosphate limitation by expressing filaments and a lethal phenotype. PLoS ONE. 2012;7(1):e30119. Epub 2012/01/19. doi: 10.1371/journal.pone.0030119. PubMed PMID: 22253901; PubMed Central PMCID: PMCPMC3258262.

160. Ramsdale M, Selway L, Stead D, Walker J, Yin Z, Nicholls SM, et al. MNL1 regulates weak acid-induced stress responses of the fungal pathogen Candida albicans. Mol Biol Cell. 2008;19(10):4393-403. Epub 2008/07/26. doi: 10.1091/mbc.E07-09-0946. PubMed PMID: 18653474; PubMed Central PMCID: PMCPMC2555942.

161. Bensen ES, Martin SJ, Li M, Berman J, Davis DA. Transcriptional profiling in Candida albicans reveals new adaptive responses to extracellular $\mathrm{pH}$ and functions for Rim101p. Mol Microbiol. 2004;54(5):1335-51. Epub 2004/11/24. doi: 10.1111/j.1365-2958.2004.04350.x. PubMed PMID: 15554973.

162. Nicholls S, Straffon M, Enjalbert B, Nantel A, Macaskill S, Whiteway M, et al. Msn2- and Msn4-like transcription factors play no obvious roles in the stress responses of the fungal pathogen Candida albicans. Eukaryot Cell. 2004;3(5):1111-23. Epub 2004/10/08. doi: 10.1128/EC.3.5.1111-1123.2004. PubMed PMID: 15470239; PubMed Central PMCID: PMCPMC522590.

163. Schweizer A, Rupp S, Taylor BN, Rollinghoff M, Schroppel K. The TEA/ATTS transcription factor CaTec1p regulates hyphal development and virulence in Candida albicans. Mol Microbiol. 2000;38(3):435-45. Epub 2000/11/09. doi: 10.1046/j.1365-2958.2000.02132.x. PubMed PMID: 11069668. 
164. Sahni N, Yi S, Daniels KJ, Huang G, Srikantha T, Soll DR. Tec1 mediates the pheromone response of the white phenotype of Candida albicans: insights into the evolution of new signal transduction pathways. PLoS Biol.

2010;8(5):e1000363. Epub 2010/05/11. doi: 10.1371/journal.pbio.1000363. PubMed PMID: 20454615; PubMed Central PMCID: PMCPMC2864266.

165. Lane S, Zhou S, Pan T, Dai Q, Liu H. The basic helix-loop-helix transcription factor Cph2 regulates hyphal development in Candida albicans partly via TEC1. Mol Cell Biol. 2001;21(19):6418-28. Epub 2001/09/05. doi:

10.1128/mcb.21.19.6418-6428.2001. PubMed PMID: 11533231; PubMed Central PMCID: PMCPMC99789.

166. Nobile CJ, Bruno VM, Richard ML, Davis DA, Mitchell AP. Genetic control of chlamydospore formation in Candida albicans. Microbiology (Reading). 2003;149(Pt 12):3629-37. Epub 2003/12/10. doi: 10.1099/mic.0.26640-0. PubMed PMID: 14663094.

167. Dixon B. The role of microvascular thrombosis in sepsis. Anaesth Intensive Care. 2004;32(5):619-29. Epub 2004/11/13. doi: 10.1177/0310057X0403200502. PubMed PMID: 15535483.

168. Singh RP, Prasad HK, Sinha I, Agarwal N, Natarajan K. Cap2-HAP complex is a critical transcriptional regulator that has dual but contrasting roles in regulation of iron homeostasis in Candida albicans. J Biol Chem. 2011;286(28):25154-70.

Epub 2011/05/20. doi: 10.1074/jbc.M111.233569. PubMed PMID: 21592964; PubMed Central PMCID: PMCPMC3137088.

169. Hickman MJ, Winston F. Heme levels switch the function of Hap1 of Saccharomyces cerevisiae between transcriptional activator and transcriptional repressor. Mol Cell Biol. 2007;27(21):7414-24. Epub 2007/09/06. doi: 10.1128/MCB.00887-07. PubMed PMID: 17785431; PubMed Central PMCID: PMCPMC2169065.

170. Maicas S, Moreno I, Nieto A, Gomez M, Sentandreu R, Valentin E. In silico analysis for transcription factors with $\mathrm{Zn}(\mathrm{II})(2) \mathrm{C}(6)$ binuclear cluster DNAbinding domains in Candida albicans. Comp Funct Genomics. 2005;6(7-8):34556. Epub 2008/07/17. doi: 10.1002/cfg.492. PubMed PMID: 18629206; PubMed Central PMCID: PMCPMC2447501.

171. Bruno VM, Mitchell AP. Large-scale gene function analysis in Candida albicans. Trends Microbiol. 2004;12(4):157-61. Epub 2004/03/31. doi: 10.1016/j.tim.2004.02.002. PubMed PMID: 15051065.

172. Bruno VM, Shetty AC, Yano J, Fidel PL, Jr., Noverr MC, Peters BM. Transcriptomic analysis of vulvovaginal candidiasis identifies a role for the NLRP3 inflammasome. mBio. 2015;6(2). Epub 2015/04/23. doi: 10.1128/mBio.00182-15. PubMed PMID: 25900651; PubMed Central PMCID: PMCPMC4453569.

173. Bruno VM, Wang Z, Marjani SL, Euskirchen GM, Martin J, Sherlock G, et al. Comprehensive annotation of the transcriptome of the human fungal pathogen Candida albicans using RNA-seq. Genome Res. 2010;20(10):1451-8. Epub 2010/09/03. doi: 10.1101/gr.109553.110. PubMed PMID: 20810668; PubMed Central PMCID: PMCPMC2945194. 
174. Liu Y, Shetty AC, Schwartz JA, Bradford LL, Xu W, Phan QT, et al. New signaling pathways govern the host response to $C$. albicans infection in various niches. Genome Res. 2015;25(5):679-89. Epub 2015/04/11. doi:

10.1101/gr.187427.114. PubMed PMID: 25858952; PubMed Central PMCID: PMCPMC4417116.

175. Khamooshi K, Sikorski P, Sun N, Calderone R, Li D. The Rbf1, Hfl1 and Dbp4 of Candida albicans regulate common as well as transcription factor-specific mitochondrial and other cell activities. BMC Genomics. 2014;15:56. Epub 2014/01/24. doi: 10.1186/1471-2164-15-56. PubMed PMID: 24450762; PubMed Central PMCID: PMCPMC3904162.

176. King JM, Kulhankova K, Stach CS, Vu BG, Salgado-Pabon W. Phenotypes and Virulence among Staphylococcus aureus USA100, USA200, USA300, USA400, and USA600 Clonal Lineages. mSphere. 2016;1(3). Epub 2016/06/16. doi: 10.1128/mSphere.00071-16. PubMed PMID: 27303750; PubMed Central PMCID: PMCPMC4899884.

177. Cogen AL, Yamasaki K, Muto J, Sanchez KM, Crotty LA, Tanios J, et al. Staphylococcus epidermidis antimicrobial delta-toxin (phenol-soluble modulingamma) cooperates with host antimicrobial peptides to kill group A Streptococcus. PLoS ONE. 2010;5(1):e8557-e. doi: 10.1371/journal.pone.0008557.

178. Lucas S. The Pharmacology of Indomethacin. Headache. 2016;56(2):436-46. Epub 2016/02/13. doi: 10.1111/head.12769. PubMed PMID: 26865183.

179. Hinson RM, Williams JA, Shacter E. Elevated interleukin 6 is induced by prostaglandin E2 in a murine model of inflammation: possible role of cyclooxygenase-2. P Natl Acad Sci USA. 1996;93(10):4885-90. Epub 1996/05/14. doi: 10.1073/pnas.93.10.4885. PubMed PMID: 8643498; PubMed Central PMCID: PMCPMC39374.

180. Aronoff DM. Cyclooxygenase inhibition in sepsis: is there life after death? Mediators Inflamm. 2012;2012:696897. Epub 2012/06/06. doi: 10.1155/2012/696897. PubMed PMID: 22665954; PubMed Central PMCID: PMCPMC3361325.

181. Wilke GA, Bubeck JW. Role of a disintegrin and metalloprotease 10 in Staphylococcus aureus alpha-hemolysin-mediated cellular injury. P Natl Acad Sci USA. 2010;107(30):13473-8. doi: 10.1073/pnas.1001815107.

182. Venugopal A. Disseminated intravascular coagulation. Indian J Anaesth. 2014;58(5):603-8. Epub 2014/12/24. doi: 10.4103/0019-5049.144666. PubMed PMID: 25535423; PubMed Central PMCID: PMCPMC4260307.

183. Gyawali B, Ramakrishna K, Dhamoon AS. Sepsis: The evolution in definition, pathophysiology, and management. SAGE Open Med. 2019;7:2050312119835043. Epub 2019/03/28. doi: 10.1177/2050312119835043. PubMed PMID: 30915218; PubMed Central PMCID: PMCPMC6429642.

184. Carestia A, Davis RP, Grosjean H, Lau MW, Jenne CN. Acetylsalicylic acid inhibits intravascular coagulation during Staphylococcus aureus-induced sepsis in mice. Blood. 2020;135(15):1281-6. Epub 2020/01/18. doi: 10.1182/blood.2019002783. PubMed PMID: 31951648. 
185. Lin MH, Lee CH, Lin C, Zou YF, Lu CH, Hsieh CH, et al. Low-Dose Aspirin for the Primary Prevention of Cardiovascular Disease in Diabetic Individuals: A Meta-Analysis of Randomized Control Trials and Trial Sequential Analysis. J Clin Med. 2019;8(5). Epub 2019/05/08. doi: 10.3390/jcm8050609. PubMed PMID: 31060297; PubMed Central PMCID: PMCPMC6572181.

186. Harper L, Balasubramanian D, Ohneck EA, Sause WE, Chapman J, Mejia-Sosa B, et al. Staphylococcus aureus Responds to the Central Metabolite Pyruvate To Regulate Virulence. mBio. 2018;9(1). Epub 2018/01/25. doi:

10.1128/mBio.02272-17. PubMed PMID: 29362239; PubMed Central PMCID: PMCPMC5784258.

187. Fourie R, Kuloyo OO, Mochochoko BM, Albertyn J, Pohl CH. Iron at the Centre of Candida albicans Interactions. Front Cell Infect Microbiol. 2018;8:185. Epub 2018/06/21. doi: 10.3389/fcimb.2018.00185. PubMed PMID: 29922600; PubMed Central PMCID: PMCPMC5996042.

188. Maguire SL, OhEigeartaigh SS, Byrne KP, Schroder MS, O'Gaora P, Wolfe KH, et al. Comparative genome analysis and gene finding in Candida species using CGOB. Mol Biol Evol. 2013;30(6):1281-91. Epub 2013/03/15. doi: 10.1093/molbev/mst042. PubMed PMID: 23486613; PubMed Central PMCID: PMCPMC3649674.

189. Fitzpatrick DA, O'Gaora P, Byrne KP, Butler G. Analysis of gene evolution and metabolic pathways using the Candida Gene Order Browser. BMC Genomics. 2010;11:290. Epub 2010/05/13. doi: 10.1186/1471-2164-11-290. PubMed PMID: 20459735; PubMed Central PMCID: PMCPMC2880306. 


\section{VITA}

Olivia Adele Todd was born in 1994 in Mobile, AL, to Jeffery and Rebecca Todd. She attended W.P. Davidson High School and graduated in 2012. Olivia followed in her father's footsteps and attended Mississippi State University. There she studied chemistry and microbiology, obtaining a Bachelor of Science degree in each subject. She graduated Summa Cum Laude in 2016. While an undergraduate she was involved in research in the lab of Dr. Todd Mlsna, investigating the use of magnetic biochar to remove contaminants from wastewater. This experience spurred an interest in research and led Olivia to enroll at the University of Tennessee Health Sciences Center in Memphis, TN, in the Microbiology, Immunology, and Biochemistry track of the Integrated Biomedical Sciences Program. She joined the lab of Dr. Brian Peters in May 2017 and began her research into fungal-bacterial interactions. Olivia anticipates graduating from the College of Graduate Health Sciences with a Doctor of Philosophy degree in June 2021. 